\title{
Multisensory cortical interactions between speech and script in fluent and dyslexic readers
}

Citation for published version (APA):

Blau, V. C. (2009). Multisensory cortical interactions between speech and script in fluent and dyslexic readers. [Doctoral Thesis, Maastricht University]. Datawyse / Universitaire Pers Maastricht. https://doi.org/10.26481/dis.20091112vb

Document status and date:

Published: 01/01/2009

DOI:

10.26481/dis.20091112vb

Document Version:

Publisher's PDF, also known as Version of record

\section{Please check the document version of this publication:}

- A submitted manuscript is the version of the article upon submission and before peer-review. There can be important differences between the submitted version and the official published version of record.

People interested in the research are advised to contact the author for the final version of the publication, or visit the DOI to the publisher's website.

- The final author version and the galley proof are versions of the publication after peer review.

- The final published version features the final layout of the paper including the volume, issue and page numbers.

Link to publication

\footnotetext{
General rights rights.

- You may freely distribute the URL identifying the publication in the public portal. please follow below link for the End User Agreement:

www.umlib.nl/taverne-license

Take down policy

If you believe that this document breaches copyright please contact us at:

repository@maastrichtuniversity.nl

providing details and we will investigate your claim.
}

Copyright and moral rights for the publications made accessible in the public portal are retained by the authors and/or other copyright owners and it is a condition of accessing publications that users recognise and abide by the legal requirements associated with these

- Users may download and print one copy of any publication from the public portal for the purpose of private study or research.

- You may not further distribute the material or use it for any profit-making activity or commercial gain

If the publication is distributed under the terms of Article $25 \mathrm{fa}$ of the Dutch Copyright Act, indicated by the "Taverne" license above, 
MULTISENSORY CORTICAL INTERACTIONS BETWEEN SPEECH AND SCRIPT IN FLUENT AND DYSLEXIC READERS

VERA CHRISTINA BLAU 
Cover design \& layout: V.Blau | K.Remke

Production:

Datawyse | Universitaire Pers Maastricht

(C) 2009 V.Blau, Maastricht

ISBN: 9789052788777

The work presented in this thesis was conducted at Maastricht University and funded by the Netherlands Organization for Scientific Research (NWO) and the Eurpean Union Sixth Framework Program (NeuroDys). 


\title{
MULTISENSORY CORTICAL INTERACTIONS BETWEEN SPEECH AND SCRIPT IN FLUENT AND DYSLEXIC READERS
}

\author{
Dissertation \\ To obtain the degree of Doctor at Maastricht University, \\ on the authority of the Rector Magnificus, Prof. dr. G.P.M.F. Mols \\ in accordance with the decision of the Board of Deans, \\ to be defended in public on November $12^{\text {th }} 2009$ at 12:00 hours. \\ by
}

Vera Christina Blau

Born August $27^{\text {th }} 1978$ in Munich

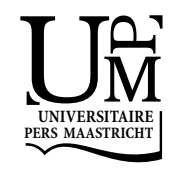




\section{Promoters}

Prof. dr. L. Blomert

Prof. dr. R. Goebel

Assessment Committee

Prof. dr. P. De Weerd

Chairman

Dr. M. Naumer

Goethe University, Frankfurt am Main, Germany

Dr. A. Sack

Prof. dr. J. Vroomen University of Tilburg, the Netherlands 
"To analyze what we do when we read would be the acme of a psychologists achievements, for it would be to describe very many of the most indicative workings of the human mind, as well as to unravel the tangled story of the most remarkable performance that civilization has learned in all its history."

Edward B. Huey, 1908 



\section{CONTENTS}

$\begin{array}{lll}\text { Chapter } 1 \quad \text { General Introduction } & 9\end{array}$

Chapter 2 Task-irrelevant visual letters interact with the processing of speech sounds in unisensory and heteromodal cortex

Chapter 4 Deviant processing of letters and speech sounds as proximate cause of reading failure: a fMRI study of dyslexic children

Chapter 5 Deviant letter-speech sound integration in dyslexia: deficit or developmental delay?

Chapter 6 General Discussion

Summary

Samenvatting

Acknowledgments

Curriculum Vitae 

Chapter 1

GENERAL INTRODUCTION

9 


\subsection{PROLOGUE}

Reading can be defined as the process of decoding meaning from written text (Gibson and Levin, 1975). It builds on the invention of a writing system through which a message is initially encoded and 'stored' on a medium. Compared to the evolution of spoken language, which is as old as the human species, the evolution of written language is a relatively recent cultural invention dating back to no more than 5500 years (Rayner and Pollatsek, 1989). Despite its short existence, writing has been portrayed as "the most remarkable specific performance that civilization has learned in all its history" (Huey, 1908). The invention of writing made it possible to convey knowledge across time and space (Snowling, 2000) in a way that was previously unattainable using transient spoken messages, and thus drastically advanced the complexity and scope of human expression. Yet, written language remains intrinsically tied to spoken language (Mattingly, 1972). Most writing systems in use today are alphabetic systems that represent the meaning of text indirectly using written symbols or letters. While letters themselves are meaningless, they bear a systematic correspondence to the distinctive speech sounds of a language, which in turn are associated with meaning when grouped to form words. The basic principle that single letters represent speech sounds is shared by all alphabetic languages and is the key to a productive writing system, in which a small number of combinable units can be used to produce an almost indefinitely large number of words (Perfetti and Marron, 1998).

Learning this basic principle of correspondence between letters and speech sounds is a critical determinant for successful reading (Ehri, 2005; Share, 1995) and has been the focus of most early reading instruction (Adams, 1994). In spite of this, empirical investigations on the role of letter-speech sound interactions in reading skill, acquisition, and disability remain scarce. This thesis examines whether the cognitive and neural process of linking letters to corresponding speech sounds is critical to reading skill, and whether variations in this foundational skill are linked to variations in reading ability. Building on findings from recent neuroimaging studies in literate adults (van Atteveldt et al., 2004; van Atteveldt et al., 2007a; van Atteveldt et al., 2007b), its broader aim is to expand the existing knowledge on cortical interactions between speech and print for typical and atypical reading development using functional magnetic resonance imaging (fMRI). Understanding the contribution of such interactions to the cortical processing of print forms a highly relevant field of research given that at least $4 \%$ of school-aged children fail to acquire fluent reading skills (Blomert, 2005; Esser et al., 1990; Shaywitz et al., 1990). A framework for understanding dyslexia will be described that connects several domains of neuroscientific research: reading (disability), multisensory processing and cognitive development. The most pertinent concepts of the various research domains will be introduced in the present chapter (Chapter 1) starting with the basic cognitive components involved in learning to read followed by theories to explain reading failure. Next, a potential framework for investigating 
dyslexia will be described that is based on its conception as multisensory processing problem. Finally, this chapter introduces the basic principles of functional magnetic resonance imaging ( $\mathrm{fMRI}$ ) and how it is a well-suited method for investigating multisensory interactions across reading development. The remaining chapters of this book (Chapter 2-5) illustrate the specific experimental studies on letter-speech sound processing that form the core of the present thesis.

\subsection{READING AND LITERACY}

Reading is a multifaceted phenomenon that can be characterized as an interplay of various kinds of skills and knowledge (Adams, 1994). It is a sensory phenomenon as well as a perceptual one, it builds on knowledge about spoken language, but starts with the processing of visual input, it draws on knowledge about syntax, semantics and contextual cues and it requires memory resources, attentional control and proper instruction. Together with the ability to write, reading constitutes the foundation for literacy, defined as the ability to identify, understand, interpret, create, communicate, compute and use printed and written materials (United Nations Educational, Scientific and Cultural Organization, UNESCO, 2004). Being literate has many economic and social advantages that affect labor quality, employment, training opportunities, and income from work. Yet, achieving high levels of literacy is by no means universal. According to estimates of the Organization for Economic and Cultural Development (OECD) approximately one- to three-quarters of adult's worldwide fail to attain literacy levels that are suitable for coping with the demands of modern life and work (OECD, 2000). Historically, being literate has been the privilege of only a few. In the Middle Ages, the ability to read and write was largely limited to priests and monks. In medieval England, being literate could even be of vital importance. Here, literacy legally entitled a person to be a 'cleric' and so they could claim 'the benefit of clergy'. This meant that a literate person could no longer be indicted in civil courts but only in ecclesiastical courts. Ecclesiastical courts had no capital punishment, which meant that being literate could at times mean as much as to avoid execution (Rogers, 2005). Fortunately, being illiterate today is typically no longer a matter of life and death. Yet, with the invention of the printing press and the expansion of literacy to all citizens via compulsory education, literacy has become a necessary skill for wider participation in civic society. Promoting literacy for the purpose of allowing everyone to achieve their goals and develop their knowledge and potentials remains one of the major goals for education (OECD, 2000). The backside of this development is that those who have difficulties attaining sufficient levels of literacy face economic and social disadvantages. Hence, gaining insights into how individuals learn to read and write, and the cognitive and neural basis of why so many struggle to learn, continues to be an important endeavor. 


\subsection{ALPHABETIC WRITING SYSTEMS}

The acquisition of literacy builds on the existence of a writing system through which spoken messages can be systematically encoded (Rogers, 2005). Some writing systems represent meaning directly via symbols (logographies), while others represent meaning in highly abstract units such as consonants and vowels (alphabets). The majority of modern languages use alphabetic scripts, such as the Roman, the Greek or the Russian alphabet. The earliest alphabetic writing system was invented about $1300 \mathrm{BC}$ in Phoenecia (now Syria and Lebanon) and was composed of 25 to 30 symbols, each corresponding to a consonantal sound. With the rise of the Greek culture representations of vowels were added to the writing system, thereby creating the basic structure of the alphabet as it is in use today (Rayner and Pollatsek, 1989). All languages using alphabetic scripts represent the sounds of spoken language -phonemes - through letters. A phoneme is defined as the minimally contrastive unit within the sounds system of language (for example the $/ \mathrm{b} /$ and $/ \mathrm{p} /$ in $b$ it and pit are single phonemes). The smallest contrastive unit of written language are graphemes, which in alphabetic languages correspond to single letters (Gleason and Ratner, 1998). However, phonemes do not consistently correspond to letters (e.g., the Dutch phoneme / $\mathrm{u}$ / is represented by the letter combination "oe"). Because of this ambiguity, the present thesis uses the simplified term speech sound to refer to the distinctive unit of spoken language that corresponds to a single letter ${ }^{1}$. Importantly, alphabetic scripts are characterized by the systematic correspondence between letters and speech sounds. This directness of this correspondence varies between different languages, from unambiguous mappings in transparent languages (e.g., Finnish) to more ambiguous mappings in opaque languages (e.g., English). In spite of these differences, however, the access to pronunciations in all alphabetic languages is likely to be governed by 'rule-like' principles that reflect the basic correspondence between letters and speech sounds (Harris and Butterworth, 2002).

Chinese, by contrast, uses a morphosyllabic writing system in which a written character maps onto a single syllable morpheme in spoken language (Perfetti et al., 2005). The greater emphasis on syllables as opposed to phonemes goes at the cost of requiring much more syllables to be memorized. For example, alphabetic writing systems such as English require 26 letters to be memorized, German 30, French 31, and Russian 33. By comparison, modern-day usage of Chinese requires some 420 distinct syllables that map onto 4.574 characters to accommodate colloquial communication and nearly ten times as many for scientific texts (Rayner and Pollatsek, 1989). Thus alphabetic writing systems are characterized by a remarkable combinatorial power and economy in their use resulting from the basic principle

\footnotetext{
${ }^{1}$ Technically, a speech sound or phone is defined as an acoustic variation in spoken language that does not signal contrastive meaning, such as the difference between the aspirated $\left[k^{h}\right]$ and unaspirated [k] (see Gleason \& Ratner, 1998).
} 
that letters can be mapped onto speech sounds. These different design features of writing systems have important implications for literacy acquisition in different languages. In Chinese, many syllables and even more characters have to be memorized, which is effortful and time consuming. To read an alphabetic script, a child must recognize that a limited number of letters represent the speech sounds of spoken language (the alphabetic principle) (Bradley and Bryant, 1978; Bradley and Bryant, 1983; Byrne et al., 1996; Ehri, 2005; Share, 1995). Once letter-speech sound associations are known, all words already present in their spoken lexicons can readily be accessed (Ziegler and Goswami, 2005) and an almost infinite number of words can be encoded as well as created (Perfetti and Marron, 1998). This makes the acquisition of letter-speech sound associations a crucial step in literacy acquisition in alphabetic languages and the focus of early reading instruction (Ehri, 2005). Despite the combinatorial power of alphabetic systems, however, understanding how speech sounds are encoded by letters in alphabetic languages is not a trivial task. Dutch school-aged children need at least one year of explicit instruction to learn which letter corresponds to which speech sound, and several more years to fully automate these taught associations (Blomert and Vaessen, 2009). One possible reason for this is the nature of spoken language development, on which reading places 'unusual' demands.

\subsection{READING: A CENTRAL ROLE FOR PHONOLOGY}

Children are experts in understanding and using spoken language long before written language becomes relevant (Gibson and Levin, 1975). Between one and six years of age children acquire more than 14000 words (Dollaghan, 1994). During and beyond this time children gradually develop an understanding that spoken language can be divided into smaller segments, such as sentences into words, and words into syllables (syllabic awareness), and syllables into sounds or phonemes (phonemic awareness). The understanding of the compositional structure of language at all levels of linguistic complexity (from whole words to single speech sounds) has been termed "phonological awareness" (Liberman and Shankweiler, 1987; Liberman et al., 1974; Liberman et al., 1989; Stanovich, 1984). Phonological awareness is often described as the conditio sine qua non for becoming a successful reader (Goswami, 2009; Share, 1995). This proposition is supported by longitudinal studies showing that phonological awareness skills predict reading achievements (Bradley and Bryant, 1983; Torgesen et al., 1994). Learning to read an alphabetic language places particular emphasis on the development of phonemic awareness ${ }^{2}$, because the understanding of print in alphabetic languages rests on the systematic

\footnotetext{
${ }^{2}$ Throughout this thesis, the term phonological awareness will be used as an umbrella term to refer to the awareness for speech structure any level of linguistic complexity, while the term phonemic awareness will be used to specifically refer to the awareness for single phonemes.
} 
correspondence of single letters to speech sounds. Phonemic awareness refers to the ability to hear, identify, and manipulate the individual phonemes in spoken words (Mattingly, 1972). Yet, phonemes are most likely not part of the representational repertoire that characterizes spoken language development before the start of formal reading instruction. Studies of speech perception in infants suggest that the lexical system is initially set up on the basis of information such as prosody, onsets, and duration (e.g., Jusczyk, 1999; Trehub et al., 1987), all of which aid syllable extraction. Thus, syllables and not phonemes might be the relevant units of spoken language development before the start of formal reading instruction (Ziegler and Goswami, 2005). This proposition is supported by studies showing that people who never learned to read exhibit a striking lack of awareness for phonemes in their language (Morais et al., 1987). Moreover, preschoolers find it very difficult to perform phonemic awareness tasks, such as indicating whether the words dog and mad share the sound /d/ (Liberman et al., 1974). The cognitive challenge for the developing reader may be to further restructure spoken language representations once reading instruction starts so that even sub-syllabic units and ultimately phonemes can be represented (Ehri, 1992). Thus, phonemic awareness might develop in response to reading instruction (Ziegler and Goswami, 2005). Only then, a child can learn how letters map onto speech sounds, which is the basis for skilled reading in alphabetic scripts. Nevertheless, certain levels of phonological awareness critical for learning to read might precede the start of formal reading instruction (Lundberg et al., 1991; Olofsson and Lundberg, 1983). Whether such preschool phonological awareness skills are confined to higher levels of linguistic complexity, such as syllables or rhymes (Goswami and Bryant, 1990) or can include initial knowledge about phonemes (Hulme et al., 1998; Muter et al., 1998), remains a matter of debate. In spite of this, good consensus exists that phonology and individual differences in phonology are critical for understanding individual differences in learning to read (also see 1.6) and that the developmental relationship between reading acquisition and phonological awareness is reciprocal (Ehri, 2005; Perfetti et al., 1987a; Torgesen et al., 1994; Wagner and Torgesen, 1987; Ziegler and Goswami, 2005). High levels of phonological awareness facilitate reading development and, at the same time, successful reading development reinforces the acquisition of phonological awareness skills (Perfetti et al., 1987b). A critical question then is: which mechanism mediates the relation between phonological awareness and learning to read?

On theoretical grounds, it has been suggested that phonological awareness skills relate to reading through letter-speech sound associations (Ehri, 2005; Goswami, 2009; Ziegler and Goswami, 2005) (also see 1.6). Cross-sectional behavioral investigations show that, in a transparent language such as Dutch, the majority of schoolaged children learn the association between letters and speech sounds successfully within one year of reading instruction (Blomert \& Vaessen, 2009). However, behavioral studies alone may not be conclusive on the nature of letter-speech sound processing during reading, as similar behavioral response patterns can arise from 
very different neural mechanisms. Techniques such as $\mathrm{fMRI}$ or electroencephalography (EEG) provide a window into the living brain that can complement findings from behavioral investigations. The usefulness of these approaches has been demonstrated over the past two decades in which a rising number of neuroimaging and electrophysiological studies have helped to elucidate component processes of reading linked to particular cortical regions and temporal processing sequences (Schlaggar and McCandliss, 2007).

\subsection{READING: MERGING PRINT AND SPEECH IN THE BRAIN}

Neuroimaging techniques have also proven to be a highly useful tool in exploring how the human brain processes the association between letters and speech sounds (Raij et al., 2000; van Atteveldt et al., 2004; van Atteveldt et al., 2007a). In one of the first studies of its kind, van Atteveldt and colleagues (2004) presented college students with letters and speech sounds in unisensory or multisensory conditions. In the multisensory trials, auditory and visual stimuli were either congruent or incongruent and the subjects' task was to passively attend the stimuli. Their results show that the processing of congruent vs. incongruent letter-speech sound pairs enhances cortical activity in superior temporal sulcus (STS)/gyrus (STG) and auditory cortex (Heschl Sulcus, HS; Planum Temporale, PT) indicating the modulation of neural responses specific for taught audiovisual associations (van Atteveldt et al., 2004). Because of the absence of a response to visual letters in HS/PT but equal responsivity to both unisensory conditions in STS/STG, the authors propose a neurofunctional model in which letters and speech sounds are integrated in STS/STG and information about the congruency between letters and speech sounds is fed back to downstream auditory cortex. Subsequently, the processing of speech sounds in HS/PT is enhanced for congruent letter-speech sound pairs or suppressed for incongruent pairs. Such a neurofunctional model is analogous to models on audiovisual processing of speech (Calvert et al., 1999; Calvert et al., 2001), suggesting that the processing of culturally defined associations might rely on similar neural structures that those for integrating more natural audiovisual events. These findings are complemented by electrophysiological studies that tax the time course of letter-speech sound integration in literate adults (Raij et al., 2000; Froyen et al., 2008; Proverbio et al., 2004). These studies indicate that the integration between letters and speech sounds can reliably be observed in the literate adult brain and suggest the recruitment of unisensory and heteromodal cortex in presumably hierarchical order. Importantly, such investigations have recently been extended to the study of developmental differences in letter-speech sound processing using event-related potentials (ERPs) (Froyen et al., 2009). Froyen and colleagues (2009) used a pre-attentive auditory ERP component (mismatch negativity or MMN) to illustrate that beginning readers after one year of reading instruction show no signs of early letter-speech sound integration. By comparison, advanced readers after four years of reading instruction did show letter-speech sound integration in the 
early time window of the MMN, but even their response was still not fully 'adultlike'. Thus, the development of letter-speech sound associations may readily be observed at the neural level across an extended developmental period.

It should be noted that more complex stimuli and tasks have also been used to study the integration of orthographic and phonological inputs in the literate brain, although not directly at the level of letters and speech sounds. Typically, these studies have used visually presented pseudowords (pronounceable nonwords) in tasks involving rhyme judgments (Booth et al., 2002a; Booth et al., 2004; Pugh et al., 2000; Pugh et al., 2001; Shaywitz et al., 2002), syllable counting judgments (Poldrack et al., 1999), or homophone decisions (Rumsey et al., 1997). Using such tasks, activation has been reported in the left inferior frontal gyrus (IFG) and the posterior part of the STS/STG. Some of these findings (e.g., Booth et al., 2004) bear an interesting resemblance with previously reported effects for the integration of basic letter-speech sound pairs (van Atteveldt et al., 2004). However, it is not clear to what degree rather complex phonological tasks tap into the neural mechanisms for representing the most basic orthographic and phonological stimuli (letters, speech sounds) and their combinations. Yet, this knowledge is critical for understanding the development of fluent reading skills (Ehri, 2005). Frontal activations, for example, which are more commonly reported using phonological manipulations, could be related to the segmentation process in speech perception or alternatively to the working memory requirements necessary to perform such tasks (Burton et al., 2000). Paradigms that rely on stimulus-manipulations while keeping task-demands low have the advantage that they might be more closely related to perceptual or 'default' cortical processes rather than processes related to performance on the task (van Atteveldt et al., 2004; van Atteveldt et al., 2007a). This might be especially relevant when studying impaired readers as their performance on phonological tasks is known to be highly variable and poor compared to that of a control group. Passive task designs on letter-speech sound integration have further shown to be statistically more powerful as a result of the blocked nature of stimulus presentation (van Atteveldt et al., 2007b). On the other hand, passive task designs have the disadvantage that they do not provide information on the contribution of neural effects on behavioral responses and hence raise the question whether neural integration effects reflect merely an epiphenomenon of seeing those particular stimuli during scanning or a behaviorally relevant process. The designs of the experiments presented in this thesis represent a tradeoff between those aspects. Chapter 2 uses an active task design in order to validate previously reported effects of letter-speech sound integration in the literate adult brain. The remaining chapters of this thesis use passive and pseudopassive designs to address the question whether the neural integration of letters and speech sounds in the brain of dyslexic readers differs from that in fluent readers. The next paragraph introduces the basic concepts relevant for understanding reading disorders. 


\subsection{DEVELOPMENTAL DYSLEXIA}

Reading disorders can be subdivided into developmental (based on heritability) and acquired types (caused by brain trauma). The present thesis focused exclusively on the developmental variant, which hereafter will be referred to as "dyslexia". Dyslexia finds its first official mentioning as a reading disorder in the British Medical Journal in November 1896 when the physician W. Pringle Morgan reported the case of a 14 year old boy who would not learn to read despite seemingly normal intellect (Morgan, 1896). This scientific report was titled "congenital word blindness" reflecting the belief that reading difficulties were in essence visual perception problems. Today dyslexia is commonly seen as a deficit in the phonological component of language (Vellutino, 1978; Vellutino et al., 2004, also see 1.1). Reading problems in dyslexia are often unexpected in relation to other cognitive abilities and the provision of effective classroom instruction. They are characterized by difficulties with accurate and/or fluent word recognition and by poor spelling and decoding abilities (Lyon et al., 2003). Epidemiological estimates indicate that dyslexia affects at least $4 \%$ of the population in the Netherlands (Blomert, 2005; Shaywitz et al., 1990), which corresponds to at least 640000 individuals. Although various other factors may play a role as well, including rapid auditory processing deficits (Tallal et al., 1993; Temple et al., 2000), visual and magnocellular deficits (Livingstone et al., 1991; Lovegrove et al., 1990; Stein and Walsh, 1997), and cerebellar deficits (Nicolson and Fawcett, 1990; Nicolson et al., 2001), impairments on phonological awareness tasks, and in particular tasks probing phonemic awareness, constitute the most common behavioral explanation for reading failure (Vellutino, 1978; Vellutino et al., 2004). As described previously, phonemic awareness refers to the ability to perceive, identify, and manipulate speech sounds within spoken words (Goswami and Bryant, 1990; Mattingly, 1972). Variations in reading performance may result from variations in any of those components. As a result, neuroimaging studies investigating phonemic awareness are also very diverse ranging from categorical perception tasks to phoneme deletion or rhyming (e.g., Dufor et al., 2007; Horwitz et al., 1998; Ruff et al., 2003; Shaywitz et al., 2002). Electrophysiological evidence complements these findings to indicate that phonological problems in dyslexia relate to deficits in the implicit phonological processing of speech (Bonte et al., 2007). Because learning to read ultimately requires mapping sounds onto letters, every variation in understanding the speech structure of language that is functionally relevant for reading should ultimately be reflected in the way letter-speech sound associations are perceived, represented or used. In that, letter-speech sound associations reflect the most proximate cause of reading failure.

Behavioral studies provide initial evidence for altered letter-speech sound conversion in dyslexic subjects. Snowling (1980) reported that recognition performance in dyslexic children was significantly reduced in an audiovisual conversion task on nonwords. This effect was particularly pronounced when a visually presented 
nonword had to be recognized in the auditory modality. Moreover, dyslexic readers showed no significant improvement on the task as a function of reading age, while fluent readers did. Using a similar paradigm, Fox (1994) found that dyslexic readers were particularly error-prone and slower in the audiovisual judgments involving nonwords as opposed to the same the unisensory conditions. Another line of evidence for the relevance of letter-speech sound associations in dyslexia comes from training studies showing that the explicit practice on letter-speech sound correspondence rules promotes reading skills in dyslexic individuals, beyond what can be achieved by using phonological training alone (Simos et al., 2002).

While initial behavioral evidence has been gathered indicating the potential existence of a letter-speech sound integration deficit in dyslexia, neuroimaging evidence is rare. Previous neuroimaging studies in dyslexia have largely focused on reading-related phonological task to investigate reading failure, such as pseudoword reading (Brunswick et al., 1999; Horwitz et al., 1998; Paulesu et al., 2001; Rumsey, 1992; Temple, 2002) or rhyming (Cao et al., 2006; Temple et al., 2001). These studies converge on the finding of underactivation in temporoparietal cortex in dyslexia (for review see Maisog et al., 2008). In particular, the angular gyrus has classically been implicated in dyslexia (for review see Price, 2001; Pugh et al., 2001) associated with semantic processing of print and speech (e.g., Binder et al., 2003). Recent fMRI studies using visual rhyming task support this conclusion by demonstrating activation in the angular gyrus for the mapping of orthography to phonology at the lexical (i.e., word) level (Booth et al., 2002b; Booth et al., 2004). In addition, reduced neural activation in the left occipitotemporal cortex, including the fusiform gyrus, has been found in dyslexia (Brunswick et al., 1999; McCrory et al., 2005; Salmelin et al., 1996; Shaywitz et al., 2002). By comparison, the IFG has been found to show increased activation in dyslexic compared to control subjects, which has been attributed the greater use of effortful compensatory strategies in dyslexia (Brunswick et al., 1999). Thus, the neurofunctional mechanisms subserving letter-speech sound integration in dyslexia remain to be explored. This thesis proposes a multisensory framework for studying dyslexia that allows investigating these questions.

\subsection{A MULTISENSORY FRAMEWORK FOR STUDYING DYSLEXIA}

As mentioned previously, learning to read an alphabetic language requires learning the associations between visual letters and speech sounds. It can therefore be conceptualized as an essentially multisensory task, in which visual symbols of print have to be associated with auditory units of speech (Snowling, 2000). Multisensory integration refers to the merging of sensory information from different modalities for the purpose of creating a coherent percept of the environment. It leads to marked improvements in the detection and identification of external events (Meredith and Stein, 1986; Stein and Meredith, 1986; Welch and Warren, 1980), in particular under noisy conditions (Sumby and Pollack, 1954). Environmental events 
from different modalities tend to complement rather than contradict each other. For example, the sight of a car is highly likely to be accompanied by its sound; the face of a particular person with a particular voice etc. Neural systems presumably use such redundancies across sensory modalities in order to increase the reliability of perception by reducing the variance associated with sensory estimates (Ernst and Buelthoff, 2004). On the behavioral level, this is reflected in faster reaction times to congruent than incongruent multisensory inputs and/or input from only one modality (Hughes et al., 1994; Miller, 1982). Using fMRI, congruency effects have also been demonstrated for the integration of audiovisual speech (Calvert et al., 1999; Calvert et al., 2000; Wright et al., 2003) objects (Amedi et al., 2005; Hein et al., 2007) and, importantly, letter-speech sound associations (Raij et al., 2000; van Atteveldt et al., 2004). Congruency effects reflect differences in the learned audiovisual associations between stimuli from different sensory modalities. Such systematic relationships between multisensory events, however, have to be learned. Hence a multisensory conceptualization of dyslexia posits that reading difficulties manifest themselves when auditory codes for processing speech sounds have to be coupled to visual codes for processing alphabetic letters. Importantly, this does not imply that the primary cause for reading difficulties necessarily also lies in the multisensory domain. In other words, a multisensory integration deficit is assumed to represent the most proximate but not necessarily primary cause for reading failure.

Approaching the study of reading disabilities from a multisensory perspective may help to solve a longstanding contradiction in reading research, namely how a deficit in the auditory-phonological domain translates into a problem in the fast recognition of visual words. This may and should be achieved at many levels of the processing hierarchy, but letters and speech sounds provide a fundamental and clearly motivated starting point. In addition, a multisensory conceptualization of reading problems may contribute to a better understanding of typical as well as atypical reading development. As mentioned earlier, children are experts at using spoken language long before written language becomes relevant and dyslexia is a disorder specific to learning to read, i.e., dyslexia can only be diagnosed after the start of reading instruction. Hence, some aspects about linking orthography to phonology, and hence visual information to auditory information, must play a critical role in explaining reading failure. This cannot be explained within a framework that investigates reading from a unisensory perspective alone. Finally, a multisensory framework offers the possibility to study the contributions of both multisensory and unisensory processes because the identification of multisensory interaction effects commonly relates multisensory to unisensory responses. In that, it may provide a better understanding of the interactive contribution of visual and auditory processes to reading failure. Because of its superior spatial resolution, fMRI provides a suitable technique in order to explore which neural structures are relevant for linking letters to speech sounds in the literate and dyslexic brain as well as adults and in children. 


\subsection{FUNCTIONAL MAGNETIC RESONANCE IMAGING}

Functional magnetic resonance imaging (fMRI) allows measuring cortical activity in vivo while a person is engaged in a cognitive task. The absence of harmful radiation makes it not only possible to measure repeatedly, but also allows studying cognitive processes in children and over the course of development. When neurons are stimulated through an electrical or chemical signal their need for energy in the form of sugar (glucose) and oxygen increases. As a result, blood flow increases in the active brain regions. This causes a temporary change in the ratio of oxygentated to deoxygenated blood, which can be capitalized using MR imaging, and is referred to as "blood oxygenation level- dependent (BOLD)" effect. Because the hemoglobin of deoxygenated blood is paramagnetic, an increase in local blood flow and a corresponding decrease in deoxyhemoblobin will lead to a stronger signal (Bandettini et al., 2000). This effect is slow compared to the neuronal response itself, typically peaking several seconds later. This poses a limit on the temporal resolution that can be achieved using $\mathrm{FMRI}$ as opposed to electrophysiological methods (EEG, MEG). Thus, fMRI measures not the neuronal response itself, but rather the metabolic demand of a brain region for oxygen and glucose.

When a person enters the MRI scanner the body is exposed to a strong magnetic field. For a typical scanner the strength of that field is 1.5 or 3 Tesla (for comparison: the earths' magnetic field has 0.00005 Tesla). Bodies are made up of atoms and most atoms possess a spin that is critical for MR imaging. The most commonly studied isotope in human MRI is hydrogen $\left({ }^{1} \mathrm{H}\right)$, which is very abundant in the human body. When hydrogen protons are placed into a magnetic field, they align themselves in the direction of the magnetic field and act as tiny magnets (the net effect is referred to as longitudinal magnetization). This in turn results in a net signal that is large enough to measure. Protons can emit or absorb energy when it matches their resonance frequency (Lamor frequency). When a radiofrequency pulse (usually 90 degrees) is applied protons are excited and shift into a higher energy state (the net effect is referred to as transversal magnetization). Once that pulse is switched off again, protons will slowly return to their original low energy state, thereby emitting radio-frequency energy that can be picked up by an antenna (Huettel et al., 2003). Several time constants are associated with this relaxation process ( $\mathrm{T} 1, \mathrm{~T} 2$, and $\mathrm{T} 2 *), \mathrm{T} 2 *$ being the most relevant for fMRI. It refers to the decay time of the signal in the presence of local field inhomogenities. T2* decays faster the more deoxygenated hemoglobin is present in a certain patch of tissue, thereby contributing to signal loss. For the spatial encoding of the signal in threedimensional (3D) space, several gradient pulses have to be applied in addition to the 90 degree pulse. These gradients induce small perturbations in the main magnetic field in each spatial direction $(x, y, z)$, so that each volume unit can be uniquely identified. Frequency and phase information encoded in this way can be transformed into a spatial image using inverse Fourier transformation. Thus, the spatial resolution of $\mathrm{fMRI}$ is relatively high (usually in the order of 3-5 $\mathrm{mm}$ ) compared to 
other non-invasive brain imaging methods (Huettel et al., 2003). Most imaging studies, including those of the present thesis, use echo-planar imaging (EPI) in which the time course of the BOLD response is measured at fast sampling rates making it possible to acquire images for the whole brain in several seconds. As discussed previously, direct investigations of the neuroanatomical substrates of letter-speech sound associations are currently rare, despite its suggested importance in learning to read. The choice for using fMRI as a method for investigation in the present thesis was based on a) its high spatial resolution that allowed us to investigate the neuroanatomy of letter-speech sound integration, b) its noninvasiveness and possibility for repeated measure and c) its feasibility for studying both the mature and the developing brain.

\subsection{SPECIFIC AIMS AND OUTLINE OF THE THESIS}

The global aim of this thesis is to broaden the existing knowledge on the neurofunctional role of letter-speech sound integration for typical and atypical reading development. We investigated the neural mechanisms for letter-speech sound integration in fluent readers and then extended it to the study of reading disorders in adults and children. Chapter 2 addresses the question whether earlier reported neural integration effects between letters and speech sounds (Van Atteveldt et al., 2004 ; 2007) reflect behaviorally relevant phenomena or are merely an epiphenomenon of seeing letter-sound pairs during scanning. More specifically, we examined whether simple unisensory decisions on speech sounds would be influenced by implicit processing of visual letters. If such effects would be found, they would indicate a behaviorally relevant and automatic function of letter-speech sound integration in the literate brain. In order to further validate the contribution of neural responses for behavioral decisions, we parametrically varied the stimulus quality of the letter. It was predicted that parametric effects from vision on audition would be observed in auditory brain regions as well as in behavioral identification of speech sounds, indicating that these effects are tightly coupled. Chapter 3 addresses the question whether letter-speech sound integration also plays a critical role in more complex cognitive processes involved in reading. We predicted that adult dyslexic subjects would differ in their neural integration of letters and speech sounds and that this would be directly related to their reading performance. Chapter 4 addresses the question whether a deficit in integrating letters and speech sounds is already present in early stages of reading acquisition ( $2^{\text {nd }}$ grade elementary school), and thus represents a fundamental variable in explaining reading difficulties as opposed to a lifetime of reading struggle. We hypothesized that the reduced neural integration of letters and speech sounds in dyslexia would already be present in elementary school children, indicating that it is an emergent property of learning to read. In Chapter 5 we further examine the nature of the proposed letter-sound integration problem. Specifically, we investigate whether the differential effect of letter-speech sound integration remains even if dyslexic 
adult subjects are matched in reading performance to a younger control group. This allowed us to investigate whether differences in neural integration between dyslexic and control readers reflect a developmental delay in reading skill acquisition or a deficit related to dyslexia itself. Finally, Chapter 6 integrates the results from previous chapters and discusses their implications for understanding the cognitive process of skilled reading and the factors contributing to reading failure. 


\section{REFERENCES}

Adams MJ. Beginning to Read: Thinking and Leaning about Print. Vol 1. Cambridge: MIT Press, 1994.

Amedi A, von Kriegstein K, van Atteveldt NM, Beauchamp MS, Naumer MJ. Functional imaging of human crossmodal identification and object recognition. Exp Brain Res 2005; 166: 559-71.

Bandettini PA, Birn RM, Donahue KM. Functional MRI: background, methodology, limits and implementation. In: Cacioppo JT, Tassinary LG and Berntson GG, editors. Handbook of Psychophysiology. Cambridge: University Press, 2000.

Binder JR, McKiernan KA, Parsons ME, Westbury CF, Possing ET, Kaufman JN, et al. Neural correlates of lexical access during visual word recognition. J Cogn Neurosci 2003; 15: 372-93.

Blomert L. Dyslexie in Nederland. Amsterdam: Uitgeverij Nieuwezijds, 2005.

Blomert L, Vaessen A. 3DM Differential Diagnostics for Dyslexia: Cognitive Analysis of Reading and Spelling. Amsterdam: Boom Test Publishers, 2009.

Booth JR, Burman DD, Meyer JR, Gitelman DR, Parrish TB, Mesulam MM. Functional anatomy of intra- and crossmodal lexical tasks. Neuroimage 2002a; 16: 7-22.

Booth JR, Burman DD, Meyer JR, Gitelman DR, Parrish TB, Mesulam MM. Modality independence of word comprehension. Hum Brain Mapp 2002b; 16: 251-61.

Booth JR, Burman DD, Meyer JR, Gitelman DR, Parrish TB, Mesulam MM. Development of brain mechanisms for processing orthographic and phonologic representations. J Cogn Neurosci 2004; 16: 1234-49.

Bradley L, Bryant PE. Difficulties in auditory organisation as a possible cause of reading backwardness. Nature 1978; 271: 746-7.

Bradley L, Bryant PE. Categorizing sounds and learning to read - a causal connection. Nature 1983; 301: 419-21.

Brunswick N, McCrory E, Price CJ, Frith CD, Frith U. Explicit and implicit processing of words and pseudowords by adult developmental dyslexics: A search for Wernicke's Wortschatz? Brain 1999; 122 ( Pt 10): 1901-17.

Burton MW, Small SL, Blumstein SE. The role of segmentation in phonological processing: an fMRI investigation. J Cogn Neurosci 2000; 12: 679-90.

Byrne ME, Crowe TA, Hale ST, Meek EE, Epps D. Metalinguistic and pragmatic abilities of participants in adult literacy programs. J Commun Disord 1996; 29: 37-49.

Calvert GA, Brammer MJ, Bullmore ET, Campbell R, Iversen SD, David AS. Response amplification in sensory-specific cortices during crossmodal binding. Neuroreport 1999; 10: 2619-23.

Calvert GA, Campbell R, Brammer MJ. Evidence from functional magnetic resonance imaging of crossmodal binding in the human heteromodal cortex. Curr Biol 2000; 10: 649-57.

Calvert GA, Hansen PC, Iversen SD, Brammer MJ. Detection of audio-visual integration sites in humans by application of electrophysiological criteria to the BOLD effect. Neuroimage 2001; 14: 427-38.

Cao F, Bitan T, Chou TL, Burman DD, Booth JR. Deficient orthographic and phonological representations in children with dyslexia revealed by brain activation patterns. J Child Psychol Psychiatry 2006; 47: 1041-50.

Dollaghan CA. Children's phonological neighbourhoods: half empty or half full? J Child Lang 1994; 21: 257-71.

Dufor O, Serniclaes W, Sprenger-Charolles L, Demonet JF. Top-down processes during auditory phoneme categorization in dyslexia: a PET study. Neuroimage 2007; 34: 1692-707.

Ehri LC. Reconceptualizing the development of sight word reading and its relationship to recoding. In: Gough LE, Ehri LC and Treiman R, editors. Reading Acquisition. Hillsdale, NJ: Erlbaum Publishers, 1992. 
Ehri LC. Development of sight word reading:phases and findings. In: Snowling MJ and Hulme C, editors. The science of reading: a handbook. Oxford: Blackwell Publishing, 2005: 135-45.

Ernst MO, Buelthoff HH. Merging the senses into a robust percept. Trends Cogn Sci 2004; 8: $162-$ 69.

Esser G, Schmidt MH, Woerner W. Epidemiology and course of psychiatric disorders in school-age children--results of a longitudinal study. J Child Psychol Psychiatry 1990; 31: 243-63.

Fox E. Grapheme-phoneme correspondence in dyslexic and matched control readers. Br J Psychol 1994; 85 ( Pt 1): 41-53.

Froyen D, Van Atteveldt N, Bonte M, Blomert L. Crossmodal enhancement of the MMN to speechsounds indicates early and automatic integration of letters and speech-sounds. Neurosci Lett 2008; 430: 23-8.

Gibson E, Levin H. The Psychology of Reading. Cambridge: MIT Press, 1975.

Gleason JB, Ratner NB. Psycholinguistics. Orlando: Harcourt Brace College Publishers, 1998.

Goswami U. The basic processes in reading: insights from neuroscience. In: Olson DR and Torrance $\mathrm{N}$, editors. The Cambridge Handbook of Literacy. New York: Cambridge University Press, 2009.

Goswami U, Bryant PE. Phonological skills and learning to read. Hillsdale, NJ: Erlbaum Publishers, 1990.

Harris M, Butterworth G. Developmental Psychology: a student's handbook. London: Psychology Press, 2002.

Hein G, Doehrmann O, Muller NG, Kaiser J, Muckli L, Naumer MJ. Object familiarity and semantic congruency modulate responses in cortical audiovisual integration areas. J Neurosci 2007; 27: 7881-7.

Horwitz B, Rumsey JM, Donohue BC. Functional connectivity of the angular gyrus in normal reading and dyslexia. Proc Natl Acad Sci U S A 1998; 95: 8939-44.

Huettel SA, Song AW, McCarthy G. Functional Magnetic Resonance Imaging. Sunderland: Sinauer Associates, 2003.

Huey E. The psychology and pedagogy of reading. New York: Macmillan, 1908.

Hughes HC, Reuter-Lorenz PA, Nozawa G, Fendrich R. Visual-auditory interactions in sensorimotor processing: saccades versus manual responses. J Exp Psychol Hum Percept Perform 1994; 20 : 131-53.

Hulme C, Muter VV, Snowling M. Segmentation does predict early progress in learning to read better than rhyme: A reply to bryant. J Exp Child Psychol 1998; 71: 39-44.

Jusczyk PW. How infants begin to extract words from speech. Trends Cogn Sci 1999; 3: 323-328.

Liberman IY, Shankweiler DP. Phonology and the problems of learning to read and write. Memory and Learning Disabilities (Suppl) 1987: 203-224.

Liberman IY, Shankweiler DP, Fischer FW, Carter B. Explicit syllable and phoneme segmentation in the young child. Journal of Experiemental Child Psychology 1974; 18: 201-12.

Liberman IY, Shankweiler DP, Liberman AM. The alphabetic principle and leraning to read. In: Shankweiler DP and Liberman IY, editors. Phonology and Reading Diability: Solving the Reading Puzzle. IARLD Research Monograph Series. Ann Arbor: University of Michigan Press, 1989.

Livingstone MS, Rosen GD, Drislane FW, Galaburda AM. Physiological and anatomical evidence for a magnocellular defect in developmental dyslexia. Proc Natl Acad Sci U S A 1991; 88: 7943-7.

Lovegrove WJ, Garzia RP, Nicholson SB. Experimental evidence for a transient system deficit in specific reading disability. J Am Optom Assoc 1990; 61: 137-46.

Lundberg I, Frost J, Peterson OP. Phonemic awareness can be developed without readng instruction. In: Brady SA and Shankweiler DP, editors. Phonological processes in literacy: a tribute to Isabelle Liberman. Hillsdale, NJ: Erlbaum Publishers, 1991: 150-165.

Lyon GR, Shaywitz SE, Shaywitz BA. A definition of Dyslexia. Annals of Dyslexia 2003; 53: 1-14. 
Maisog JM, Einbinder ER, Flowers DL, Turkeltaub PE, Eden GF. A meta-analysis of functional neuroimaging studies of dyslexia. Ann N Y Acad Sci 2008; 1145: 237-59.

Mattingly IG. Reading, the linguistic process, and linguistic awareness. In: Kavanagh JK and Mattingly IG, editors. Language by ear and eye: The relationship between speech and reading. Cambridge: MIT Press, 1972.

McCrory EJ, Mechelli A, Frith U, Price CJ. More than words: a common neural basis for reading and naming deficits in developmental dyslexia? Brain 2005; 128: 261-7.

Meredith MA, Stein BE. Visual, auditory, and somatosensory convergence on cells in superior colliculus results in multisensory integration. J Neurophysiol 1986; 56: 640-62.

Miller J. Divided attention: evidence for coactivation with redundant signals. Cogn Psychol 1982; 14: 247-79.

Morais J, Castro SL, Scliar-Cabral L, Kolinsky R, Content A. The effects of literacy on the recognition of dichotic words. Q J Exp Psychol A 1987; 39: 451-65.

Morgan WP. A Case of Cogenital Word Blindness. British Medical Journal 1896; ii: 178.

Muter V, Hulme C, Snowling M, Taylor S. Segmentation, not rhyming, predicts early progress in learning to read. J Exp Child Psychol 1998; 71: 3-27.

Nicolson RI, Fawcett AJ. Automaticity: a new framework for dyslexia research? Cognition 1990; 35: 159-82.

Nicolson RI, Fawcett AJ, Dean P. Developmental dyslexia: the cerebellar deficit hypothesis. Trends Neurosci 2001; 24: 508-11.

OECD Organization for Economic and Cultural Development. Literacy in the Informtaion Age: Final Report of the International Adult Literacy Survey. Paris: OECD, 2000: 5-8.

Olofsson A, Lundberg I. Can phonemic awareness be trained in kindergarten? Scand J Psychol 1983; 24: 35-44.

Paulesu E, Demonet JF, Fazio F, McCrory E, Chanoine V, Brunswick N, et al. Dyslexia: cultural diversity and biological unity. Science 2001; 291: 2165-7.

Perfetti CA, Beck I, Bell L, Hughes C. Phonemic knowldege and learning to read are reciprocal: A longitudinal study of first grade children. Merill-Palmer Quarterly 1987a; 33: 283-319.

Perfetti CA, Beck I, Bell LC, Hughes C. Phonemic Knowledge and Learning to Read Are Reciprocal a Longitudinal-Study of 1st-Grade Children. Merrill-Palmer Quarterly-Journal of Developmental Psychology 1987b; 33: 283-319.

Perfetti CA, Liu Y, Tan LH. The lexical constituency model: some implications of research on Chinese for general theories of reading. Psychol Rev 2005; 112: 43-59.

Perfetti CA, Marron MA. Learning to Read: Literacy Acquisition by Children and Adults. In: Wagner DA, editor. Advances in adult literacy research and development. Cresskill: Hampton Press, 1998.

Poldrack RA, Wagner AD, Prull MW, Desmond JE, Glover GH, Gabrieli JD. Functional specialization for semantic and phonological processing in the left inferior prefrontal cortex. Neuroimage 1999; 10: 15-35.

Price CJ. Functional-imaging studies of the 19th Century neurological model of language. Rev Neurol (Paris) 2001; 157: 833-6.

Proverbio AM, Vecchi L, Zani A. From orthography to phonetics: ERP measures of grapheme-tophoneme conversion mechanisms in reading. J Cogn Neurosci 2004; 16: 301-17.

Pugh KR, Mencl WE, Jenner AR, Katz L, Frost SJ, Lee JR, et al. Functional neuroimaging studies of reading and reading disability (developmental dyslexia). Ment Retard Dev Disabil Res Rev 2000; 6: 207-13.

Pugh KR, Mencl WE, Jenner AR, Katz L, Frost SJ, Lee JR, et al. Neurobiological studies of reading and reading disability. J Commun Disord 2001; 34: 479-92.

Raij T, Uutela K, Hari R. Audiovisual integration of letters in the human brain. Neuron 2000; 28 : 617-25. 
Rayner K, Pollatsek A. The Psychology of Reading. New Jersey: Prentice-Hall, 1989.

Rogers R. Writing Systems: A Linguistic Approach. Oxford: Blackwell Publishing, 2005.

Ruff S, Marie N, Celsis P, Cardebat D, Demonet JF. Neural substrates of impaired categorical perception of phonemes in adult dyslexics: an fMRI study. Brain Cogn 2003; 53: 331-4.

Rumsey JM. Failure to activate left temporoparietal cortex in dyslexia. Archives of Neurology 1992; 49: 527-534.

Rumsey JM, Nace K, Donohue B, Wise D, Maisog JM, Andreason P. A positron emission tomographic study of impaired word recognition and phonological processing in dyslexic men. Arch Neurol 1997; 54: 562-73.

Salmelin R, Service E, Kiesila P, Uutela K, Salonen O. Impaired visual word processing in dyslexia revealed with magnetoencephalography. Ann Neurol 1996; 40: 157-62.

Schlaggar BL, McCandliss BD. Development of neural systems for reading. Annu Rev Neurosci 2007; 30: 475-503.

Share DL. Phonological recoding and self-teaching: Sine qua non of reading acquisition. Cognition 1995; 55: 151.

Shaywitz BA, Shaywitz SE, Pugh KR, Mencl WE, Fulbright RK, Skudlarski P, et al. Disruption of posterior brain systems for reading in children with developmental dyslexia. Biol Psychiatry 2002; 52: 101-10.

Shaywitz SE, Shaywitz BA, Fletcher JM, Escobar MD. Prevalence of reading disability in boys and girls. Results of the Connecticut Longitudinal Study. Jama 1990; 264: 998-1002.

Simos PG, Fletcher JM, Bergman E, Breier JI, Foorman BR, Castillo EM, et al. Dyslexia-specific brain activation profile becomes normal following successful remedial training. Neurology 2002; 58: $1203-13$.

Snowling MJ. The development of grapheme-phoneme correspondence in normal and dyslexic readers. J Exp Child Psychol 1980; 29: 294-305.

Snowling MJ. Dyslexia. Òxford: Blackwell Publishers, 2000.

Stanovich KE. Assessing phonological awareness in kindergarten children: issues of task comparability. Journal of Experimental Child Psychology 1984; 38: 175-190.

Stein BE, Meredith MA. The Merging of the Senses. Cambridge: MIT Press, 1986.

Stein J, Walsh V. To see but not to read; the magnocellular theory of dyslexia. Trends Neurosci 1997; 20: 147-52.

Sumby WH, Pollack I. Visual contribution to speech intelligibility in noise. The Journal of the Acoustic Society of America 1954; 26: 212-215.

Tallal P, Miller S, Fitch RH. Neurobiological basis of speech: a case for the preeminence of temporal processing. Ann N Y Acad Sci 1993; 682: 27-47.

Temple E. Brain mechanisms in normal and dyslexic readers. Curr Opin Neurobiol 2002; 12: 17883.

Temple E, Poldrack RA, Protopapas A, Nagarajan S, Salz T, Tallal P, et al. Disruption of the neural response to rapid acoustic stimuli in dyslexia: evidence from functional MRI. Proc Natl Acad Sci U S A 2000; 97: 13907-12.

Temple E, Poldrack RA, Salidis J, Deutsch GK, Tallal P, Merzenich MM, et al. Disrupted neural responses to phonological and orthographic processing in dyslexic children: an fMRI study. Neuroreport 2001; 12: 299-307.

Torgesen JK, Wagner RK, Rashotte CA. Longitudinal studies of phonological processing and reading. J Learn Disabil 1994; 27: 276-86; discussion 287-91.

Trehub SE, Thorpe LA, Morrongiello BA. Organizational processes in infants' perception of auditory patterns. Child Dev 1987; 58: 741-9.

UNESCO United Nations Educational, Scientific, and Cultural Organization. The Plurality of Literacy and its implications for Policies and Programs: Position Paper: UNESCO Education Sector, 2004. 
van Atteveldt N, Formisano E, Goebel R, Blomert L. Integration of letters and speech sounds in the human brain. Neuron 2004; 43: 271-82.

van Atteveldt NM, Formisano E, Blomert L, Goebel R. The effect of temporal asynchrony on the multisensory integration of letters and speech sounds. Cereb Cortex 2007a; 17: 962-74.

van Atteveldt NM, Formisano E, Goebel R, Blomert L. Top-down task effects overrule automatic multisensory responses to letter-sound pairs in auditory association cortex. Neuroimage 2007b; 36: 1345-60.

Vellutino FR. Dyslexia: Theory and Research. Cambridge: MIT Press, 1978.

Vellutino FR, Fletcher JM, Snowling MJ, Scanlon DM. Specific reading disability (dyslexia): what have we learned in the past four decades? J Child Psychol Psychiatry 2004; 45: 2-40.

Wagner $\mathrm{R}$, Torgesen $\mathrm{J}$. The nature of phonological processes and its causal role in the acquisition of reading skills. Psychological Bulletin 1987; 101: 192-212.

Welch RB, Warren DH. Immediate perceptual response to inter-sensory discrepancy. Psychological Bulletin 1980; 88: 638-667.

Wright TM, Pelphrey KA, Allison T, McKeown MJ, McCarthy G. Polysensory interactions along lateral temporal regions evoked by audiovisual speech. Cereb Cortex 2003; 13: 1034-43.

Ziegler JC, Goswami U. Reading acquisition, developmental dyslexia, and skilled reading across languages: a psycholinguistic grain size theory. Psychol Bull 2005; 131: 3-29. 



\section{TASK-IRRELEVANT VISUAL LETTERS INTERACT WITH THE PROCESSING OF SPEECH SOUNDS IN UNISENSORY AND HETEROMODAL CORTEX}

Based on:

Blau, V., van Atteveldt, N., Formisano, E., Goebel, R., Blomert, L. (2008). Taskirrelevant visual letters interact with the processing of speech sounds in unisensory and heteromodal cortex. European Journal of Neuroscience, 28, 500-509. 


\begin{abstract}
Letters and speech sounds are the basic units of correspondence between spoken and written language. Associating auditory information of speech sounds with visual information of letters is critical for learning to read; however, the neural mechanisms underlying this association remain poorly understood. The present functional magnetic resonance imaging study investigates the automaticity and behavioral relevance of integrating letters and speech sounds. Within a unisensory auditory identification task, speech sounds were presented in isolation or bimodally in congruent and incongruent combinations with visual letters. Furthermore, the quality of the visual letters was manipulated parametrically. Our analyses revealed that the presentation of congruent visual letters led to a behavioral improvement in identifying speech sounds, which was paralleled by a similar modulation of cortical responses in the left superior temporal sulcus. Under low visual noise, cortical responses in superior temporal and occipito-temporal cortex were further modulated by the congruency between auditory and visual stimuli. These crossmodal modulations of performance and cortical responses during an unisensory auditory task (speech identification) indicate the existence of a strong and automatic functional coupling between processing of letters (orthography) and speech (phonology) in the literate adult brain.
\end{abstract}




\section{INTRODUCTION}

In alphabetic scripts, learning the correspondences between the smallest units of written and spoken language (letters/graphemes vs. speech sounds/phonemes) is a key step in reading acquisition (Ehri, 2005). A failure to acquire sufficiently automated letter-speech sound associations has been proposed as a cause for dyslexia (Vellutino et al., 2004). Whereas behavioral studies suggest the fast and automatic multisensory activation from letter to sound representations (Borowsky et al., 1999; Dijkstra et al., 1993; Dijkstra et al., 1989; Frost and Katz, 1989), it is not clear which neural mechanisms subserve the influences from letters to speech sounds and its effects on behavior in literate adults. Several neuroimaging studies have investigated the neural basis of letter-to-sound conversion both at the level of word processing (Booth et al., 2002a; Booth et al., 2007; Fiebach et al., 2002; Fiez et al., 1999; Simos et al., 2002; Tagamets et al., 2000) and at the level of letters and speech sounds (Hashimoto and Sakai, 2004; Raij et al., 2000; van Atteveldt et al., 2004). Converging results indicate the involvement of heteromodal superior temporal cortex (STS/STG), auditory Heschl sulcus/Planum temporale (HS/PT) and visual occipito-temporal cortex (OT). The processing of congruent vs. incongruent letters-speech sound pairs was found to enhance cortical activity in superior temporal and auditory cortex (van Atteveldt et al., 2004), indicating the modulation of neural responses specific for learned audiovisual associations.

The aim of the present behavioral and functional magnetic resonance imaging (fMRI) study was twofold. First, we investigated whether letters influence speech processing even if visual processing is not explicitly required by the task. Second, we investigated whether the neural influences of congruent/incongruent visual stimulation on speech sound processing are relevant for the behavioral facilitation/inhibition of speech identification. The first question was motivated by behavioral and electrophysiological findings indicating that, in literate adults, the access from letter to sound representations is fast and highly automated (Dijkstra et al., 1993; Dijkstra et al., 1989; Froyen et al., 2008; Perre and Ziegler, 2008; Proverbio et al., 2004). Hence, we presented visual letters as task-irrelevant stimuli during a unisensory forced choice speech identification task. Note that analogous to earlier reports (Dijkstra et al., 1989) the term 'automatic' is used to indicate multisensory influences that occur even though the visual letter is neither directly task-relevant nor connected to the required response. Similar designs have been used in electrophysiology research to investigate the influence of task-irrelevant auditory stimulation on visual processing (Busse et al., 2005; McDonald et al., 2003). Speech sounds were presented alone or bimodally in congruent and incongruent combinations with the letter. It was predicted that letters modulate the processing of speech sounds in heteromodal (STS/STG) and/or 'modality-specific' cortices (HS/PT, OT), indicating automatic visual influences on speech identification. The second question was motivated by the observation that previous neuroimaging studies have often either used passive perception tasks (Calvert et al., 2000; Olson et al., 2002; 
van Atteveldt et al., 2004; van Atteveldt et al., 2007a) or indirect performance measures (Callan et al., 2003; Macaluso et al., 2004), concealing the contribution of audiovisual integration to behavioral response facilitation/inhibition. We addressed this issue by presenting visual letters using three degradation levels (low, medium and high visual noise), and examining the consequences of this manipulation at the behavioral and neural level. We predicted parametric influences of visual degradation not only for behavioral responses to speech sounds but also for neural activity related to letter-speech sound processing.

\section{MATERIALS AND METHODS}

\section{SUBJECTS}

Nineteen healthy, right-handed subjects (eight male) with normal hearing and normal or corrected-to-normal vision were recruited for the present fMRI experiment. All participants were native Dutch speakers enrolled in (under)graduate programs at Maastricht University (mean age: 21.4 years; SD: 3.5). The psychophysical experiment included 10 right-handed subjects with normal or correctedto-normal vision (three male) drawn from the same population of students (mean age: 20.5 years; SD: 1.65). All subjects were screened to exclude psychiatric, neurological or reading disorders. Prior to testing subjects gave informed written consent to participate. Subjects were paid for their participation, which was voluntary and in accordance with the Faculty of Psychology ethical guidelines.

\section{STIMULI}

Stimuli were visual letters and auditory speech sounds corresponding to the Dutch vowels [a] and [e]. Auditory stimuli were created by digitally recording the voice of a native Dutch female speaker at a sampling rate of $44.1 \mathrm{kHz}$ (16 bit). All recordings were resampled at $22.05 \mathrm{kHz}$ offline and had a total length of approximately 400 $\mathrm{ms}$. Speech sounds were then mixed with pink auditory noise (sampling frequency $22 \mathrm{kHz}, 16$ bit) of the same length using goldwave v5.12 (GoldWave). Subsequently, combined auditory stimuli were matched for overall intensity ( $\pm 74 \mathrm{~dB})$. Behavioral pilot-testing indicated how subjects perceived the degraded speech sounds using various noise levels $(5,10,15,20$ and $25 \mathrm{~dB})$, one of which was selected for the present $\mathrm{fMRI}$ study $(10 \mathrm{~dB})$. Visual stimuli were lower-case letters (print-type: times new roman; visual angle: 2.8 x 3.8 degrees) corresponding to vowels 'a' and 'e'. All visual letters were in a white font on a gray background. Using PaintShopPro (Corel Cooperation), noise in the visual domain was added by applying a two-step procedure: first, the overall contrast was lowered by $40 \%$ in order to remove highcontrast components that would prohibit sufficient visual masking; second, a visual mask was overlaid on top of the letter using three opacity levels. The mask was created by taking bar-shaped figures from the original low-contrast stimulus and 
randomly reassembling those shapes to create an abstract visual profile. This way visual letters were created with low, medium and high levels of noise corresponding to good, medium and low letter perceptibility (Fig. 1A).

\section{DESIGN AND TASK}

Stimuli were presented in unisensory auditory and audiovisual conditions on a trialby-trial basis within a forced-choice auditory identification task. During multisensory trials auditory and visual stimuli were presented simultaneously in either congruent or incongruent combinations (congruency factor). Auditory stimuli were presented slightly degraded (see 'Stimuli' section) to prevent behavioral ceiling effects. Additionally, the visual letter had high, medium or low quality (noise factor) resulting in a total of seven experimental conditions: unisensory auditory (A), audiovisual congruent with low amounts of visual noise (AVcon1), medium amounts of visual noise (AVcon2), and high amounts of visual noise (AVcon3). Likewise, audiovisual incongruent trials were presented with low amounts of visual noise (AVinc1), medium amounts of visual noise (AVinc2), and high amounts of visual noise (AVinc3). A psychophysical study, using an unrelated subject group, preceded the fMRI study (in the 'Results' section referred to as 'outside scanner') and consisted of two runs with 40 trials per condition resulting in a total of 280 trials in pseudorandom order, which were repeated with a delay of $400 \mathrm{~ms}$. Subjects were instructed to identify via button-press whether they perceived the speech sound /a/ or /e/. In addition to the overt auditory task, subjects were instructed to maintain fixation on the center of the screen. To maximize the assumed multisensory perceptual gain induced by visual co-stimulation during speech identification, we used a mixed experimental design, in which each experimental run contained congruent and incongruent trials presented at a ratio of $75 \%$ congruent: $25 \%$ incongruent trials in one half of the run and $75 \%$ incongruent: $25 \%$ congruent trials in the other half of the same run (no blocked presentation). The order of $75 \%$ congruent vs. $75 \%$ incongruent periods within a run was counterbalanced across subjects. Since reading starts with the processing of visual input, we focused on studying the influence from letters to speech sounds and not vice versa. 
A
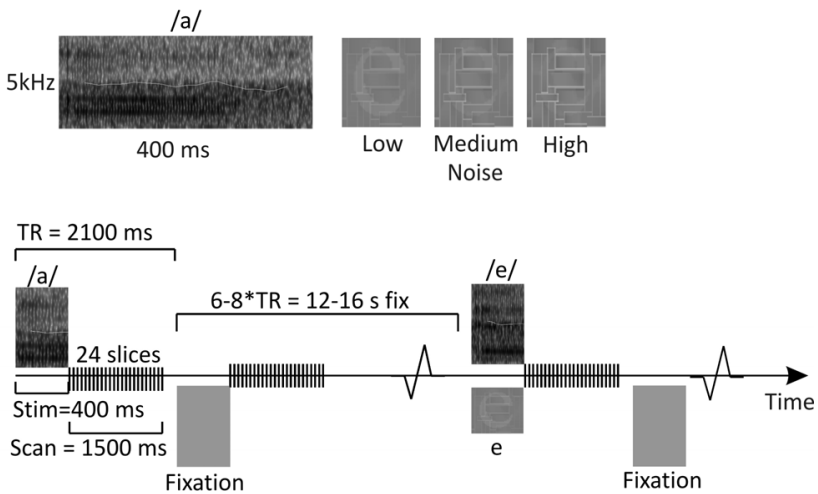

Figure 1. (A) Exemplars of experimental stimuli, auditory $/ a$ and visual 'e' masked with low, medium and high degrees of visual noise. (B) Illustration of MR experimental setup with timing parameters of four representative trials.

No external cue indicated a switch in congruent: incongruent ratio in line with subjects' consistent report of being unaware of the clustered trial structure. The necessity of this manipulation was established in a pilot experiment. Its rationale can be described as follows: the identity of the speech sounds should not be predictable by attending the visual letter only, as in the case of blocked stimulus presentation. At the same time a $50 \%$ congruent: incongruent ratio would trigger a situation in which it is unclear whether subjects will use the visual channel in order to solve the speech identification task or whether they will try to reduce its influence by ignoring it. Hence, a design investigating the perceptual gain on speech processing experienced through the presence of visual letters has to provide a clear incentive for processing visual information. This was achieved by changing the congruent: incongruent ratio to $75 \%: 25 \%$ in the present study, a situation where subjects profit from processing the visual letter for speech sound identification without making the decision predictable. To validate the current paradigm, behavioral responses between congruent trials embedded in $75 \%$ congruent vs. $75 \%$ incongruent trials were compared (and incongruent trials, respectively). No difference in accuracy of speech identification was induced as the trial ratio changed, indicating the absence of strategic task differences or global attention/arousal effects and supporting the feasibility of the current paradigm for investigating visual influences on speech identification.

The fMRI experiment, during which behavioral data were also acquired (in the 'Results' section referred to as 'inside scanner') differed from the psychophysical experiment in the following way: one session consisted of two runs with 24 trials per condition and each experimental run consisted of widely spaced single trials (68 TRs or 12-16 s). Aside from this adjustment in the temporal spacing of events, auditory speech sounds were presented slightly less degraded during scanning (8 $\mathrm{dB}$ ) to account for the auditory saturation effects created by the scanner environment. 


\section{IMAGE ACQUISITION AND DATA ANALYSIS}

Data were acquired on a 3T Siemens Allegra head scanner using gradient echo planar imaging (EPI). All participants completed two experimental runs, in which $582 \mathrm{~T}^{*}$-weighted BOLD contrast volumes were acquired for each run. Based on structural information from a previous 9-slice localizer scan, 24 axial slices were positioned in each subject to cover the auditory and visual cortices (TE $=30 \mathrm{~ms}$, TRslice = $63 \mathrm{~ms}$, matrix size: $64 \times 64 \times 24$, FOV: $192 \mathrm{~mm}^{2}$, voxel size: $3 \times 3 \times 4 \mathrm{~mm}^{3}$ ). Volume acquisition time was $1.5 \mathrm{~s}$ followed by a silent delay of $600 \mathrm{~ms}$ in which stimuli were presented resulting in a TR of $2.1 \mathrm{~s}$ (Fig. 1A). The inter-scan gap was used to minimize the effects of scanning noise on experimental activation (Jancke et al., 2002). Furthermore, the use of a long inter-trial interval (mean: 7 TR /14.7 s, jitter: $\pm 1 \mathrm{TR} / 2.1 \mathrm{~s}$ ) permitted to separate the hemodynamic response to individual trials with the advantage of modeling BOLD signal changes on a single trial basis.

A high-resolution T1-weighted anatomical image (voxel size: $1 \times 1 \times 1 \mathrm{~mm}^{3}$ ) was acquired for each subject using a three-dimensional magnetization-prepared rapid acquisition gradient echo (MP-RAGE) sequence ( $T R=2.3 \mathrm{~s}$, TE $=3.39 \mathrm{~ms}$, matrix size: 256x256, 192 slices/slab). Imaging data were analyzed using BrainVoyager QX software (Brain Innovation, Maastricht, Netherlands; Goebel et al., 2006). Functional data were preprocessed to correct for slice scan time differences (sinc interpolation), three-dimensional motion artifacts (trilinear interpolation), linear drifts and low-frequency non-linear drifts due to head motion (high pass filter $\leq 3$ cycles/time course). No spatial or additional temporal smoothing was applied. Functional data were then coregistered with the anatomical volume and transferred into standard stereotaxic space using Talairach normalization (Talairach and Tournoux, 1988). A design matrix was set up to model all experimental conditions for each acquisition. Error trials were modeled as separate predictor and excluded from further analysis.

Statistical maps were generated by modeling the evoked hemodynamic response for the different stimuli as boxcars convolved with a two-gamma hemodynamic response function in the context of the general linear model (GLM). Populationlevel inferences concerning BOLD signal changes between the experimental conditions were based on a random effects model with predictors separated for each subject. Two different GLMs were used for the present analysis. The first GLM (GLM1) was a single-factor model including all seven conditions as separate predictors. This model was used to localize brain areas that responded to both unisensory auditory and audiovisual conditions (referred to as 'conjunction analysis', see Fig. 3). Overlapping activation for unisensory and multisensory conditions is a prerequisite for investigating the additional value of adding a visual stimulus. Therefore, only voxels that were significantly activated in the conjunction analysis were included in further analysis by application of a functional mask. The conjunction was computed in the following way: [Auditory > Baseline] $n$ [Audio-Visual > Base- 
line]. In this analysis, a new statistical value is computed for each voxel as the minimum of the statistical values resulting from the two included contrasts.

Activations were considered significant after correcting for multiple comparisons using cluster-size thresholding (Forman et al., 1995; Goebel et al., 2006). This method exploits the fundamental assumption that areas of activity tend to stimulate signal changes over spatially contiguous groups of voxels rather than over sparsely isolated voxels. The computation of the minimum cluster threshold is accomplished via MonteCarlo simulation of the random process of image generation, followed by the injection of spatial correlations between neighboring voxels, voxel intensity thresholding, masking (optional) and cluster identification. Starting from a manually adjusted voxel-level probability threshold, a minimum cluster size threshold is automatically set yielding $5 \%$ (or less) protection against false positive detection at the cluster level. In the present study, an initial voxel-level (uncorrected) threshold was set to $0.001(t=4.25)$ uncorrected, resulting in a cluster-level of $160 \mathrm{~mm}^{3}$ after 1000 iterations, corresponding to a corrected false positive probability of $5 \%$ or less.

Furthermore, GLM1 was used in a post hoc analysis to investigate the effect of congruency for all three levels of visual noise separately by using the contrasts [AV1con > AV1inc], [AV2con > AV2inc] and [AV3con > AV3inc] (referred to as 'congruency analysis', Fig. 5). The correction for multiple comparisons was again carried out using cluster size thresholding. To investigate the congruency effect, we initially set an uncorrected $P$-value of $0.01(t=3.27)$, which resulted in a cluster size threshold of $149 \mathrm{~mm}^{3}$ after 1000 iterations (alpha < 0.05). The second GLM (GLM2) was a $2 \times 3$ factorial model with factors congruency (congruent, incongruent) and visual noise level (low, medium, high) in order to specifically search for interaction effects (referred to as 'interaction analysis', Fig. 4). We corrected for cluster size using an initial $p$-value of $0.01(t=3.79)$, which after 1000 iterations resulted in a cluster size threshold of $111 \mathrm{~mm}^{3}$ (alpha < 0.05). Statistical analyses for selected regions of interest in GLM1 and GLM2 were based on average percent signal change values within a region of interest (ROI). Peak percent signal change values were also used but were plotted for visualization purposes only (bar graphs: Figs 4 and 5).

\section{BEHAVIORAL RESULTS}

\section{OUTSIDE SCANNER (PSYCHOPHYSICS)}

Behavioral outcomes from the psychophysical experiment outside the scanner revealed that the identification of speech sounds was influenced by the presentation of a task-irrelevant visual letter. To assess the influence of the visual letter on speech sound identification, unisensory auditory responses were subtracted from responses in audiovisual conditions for each participant (AV-A). Positive reaction time difference scores therefore indicated disrupted speech sound identification on 
audiovisual trials vs. auditory trials, whereas negative difference scores indicated enhanced identification performance. Based on the difference scores for each multisensory condition, we found a significant congruency by visual noise interaction on reaction times for the identification of the speech sounds $\left(F_{1.42,9}=14.91, p\right.$ $<0.001$; Fig. 2, upper panel). Comparisons of congruent vs. incongruent letterspeech sound pairs revealed the strongest influence of audiovisual congruency under the condition where the letter was least degraded (AV1) $\left(t_{9}=-5.01, p<\right.$ 0.001 ) and decreasing, but still significant congruency effects for medium (AV2) ( $t_{9}$ $=-2.64, p<0.05)$ and high amounts of visual noise (AV3) $\left(t_{9}=-2.55, p<0.05\right)$.
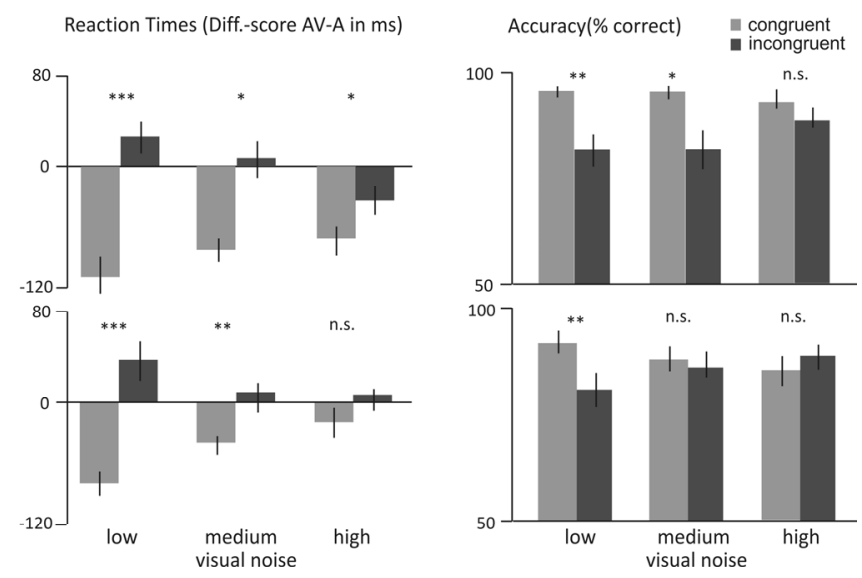

Figure 2. Bar diagrams of reaction time difference scores ([AV-A] left panel) and accuracy data (right panel) for behavioral identification of speech sounds for the two independent subject populations outside (upper panel) and inside the scanner (lower panel) reveal remarkably similar patterns of performance. Stars indicate the level of significance $\left({ }^{*} p<0.05 ;{ }^{* *} p<0.01 ;{ }^{* *} p<0.001\right)$.

Thus, subjects' identification speed of speech sounds was maximal when accompanied by a visual letter that was minimally distorted and matched the speech sound. In contrast, identification was slowest when accompanied by a minimally distorted visual letter that did not match the sound. This means that speech sound identification on audiovisual incongruent trials relative to auditory alone trials was disrupted, whereas speech sound identification on audiovisual congruent trials relative to auditory alone trials was enhanced. Both reaction time effects, enhancement and disruption, were weakened when the visual letter became more degraded. Furthermore, subjects in the behavioral study also identified speech sounds more accurately when these sounds were combined with well-perceivable letters in congruent combinations, relative to their accuracy for incongruent lettersound combinations $\left(t_{9}=3.53, p<0.01\right)$. This accuracy effect was smaller but remained significant at the medium level of noise $\left(t_{9}=2.69, p<0.05\right)$, and was absent at high amounts of noise. This pattern was reflected in a significant interaction between visual noise and letter-sound congruency $\left(F_{1.42,9}=5.88, p<0.05\right)$. 


\section{INSIDE SCANNER}

Similar to the behavioral results outside the scanner, we found a significant noise by congruency interaction effect $\left(F_{2,18}=15.6, p<0.001\right)$ during scanning. Participants responded faster to speech sounds when the sounds were accompanied by a low-noise, congruent visual letter relative to an incongruent letter $\left(t_{18}=-4.67, p<\right.$ $0.00)$. This response enhancement was also present at medium visual noise levels $\left(t_{18}=-3.47, p<0.01\right)$, but absent at high visual noise levels. In other words, at low and medium visual noise levels, congruent audiovisual trails enhanced the identification of speech sounds relative to auditory alone trials, whereas audiovisual incongruent trials disrupted speech sound identification relative to auditory alone trials. This result indicates that subjects process the visual letters effectively also during incongruent trials and, hence, discard an explanation of the congruency effect in terms of general differential attention. Moreover, letter-sound congruency and visual noise level also interacted to predict the accuracy for speech sound identification $\left(F_{1.22,18}=9.26, p<0.01\right.$; Fig. 2, lower panel). Pair-wise comparisons assessing the effect of congruency per level of visual noise showed that the effect was mainly driven by more accurate responses during congruent vs. incongruent trials when the visual letter contained low noise (AVcon1 > AVinc1) $\left(t_{18}=3.38, p<\right.$ $0.01)$. In contrast, when the visual letter contained medium or high levels of noise, congruency between letters and speech sounds did not significantly contribute to more accurate sound identification. Overall, the pattern of reaction time and accuracy effects outside the scanner were quantitatively and qualitatively similar to behavioral performance inside the scanner (Fig. 2).

\section{IMAGING RESULTS}

\section{CONJUNCTION ANALYSIS}

Figure 3 provides an overview of brain areas activated by unisensory and multisensory stimulation, as revealed by the conjunction analysis [Auditory $>$ Baseline] $n$ [AudioVisual > Baseline]. Only those voxels that were significantly activated in this analysis were included in further analysis. Our goal was to first compare the global pattern of activation to previous passive (van Atteveldt et al., 2004, 2007) studies on letter-sound integration. Secondly, the degree of overlapping activation for unisensory auditory stimulation and audiovisual stimulation is a first indication for potential 'additional' activation changes induced by the visual stimulus. We found areas of activation along the middle portions of the STG/STS extending into the middle temporal gyrus (MTG) bilaterally. Furthermore, activated brain regions included HS/PT in the left hemisphere and visual striate and extrastriate brain regions bilaterally, pre- and postcentral gyri and inferior parietal cortex bilaterally as well as basal ganglia, insular, putamen and left anterior cingulate cortex (Fig. 3). 


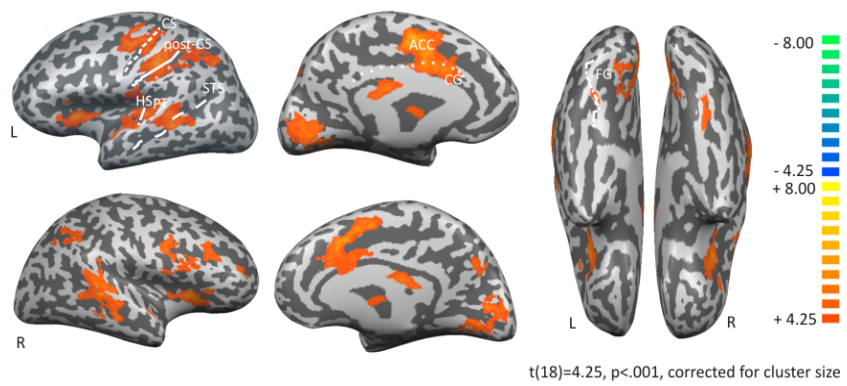

Figure 3. Group results for the conjunction analysis (cluster size corrected at alpha $<0.05$ ) projected on inflated cortical surface of one representative subject. Lateral (A), medial (B) and ventral (C) projections depict overlapping regions between auditory and audiovisual conditions. CG, cingulate gyrus; CS, central sulcus; FG,fusiform gyrus; HS, Heschl sulcus; PT, planum temporale; STS, superior temporal sulcus.

\section{INTERACTION ANALYSIS}

The second step in the analysis was to directly assess how the visual noise level manipulation influenced speech sound processing, and to assess potential interactions between congruency and noise level via a 2x3-factorial GLM (GLM2, see Materials and methods). No main effects of visual noise level or congruency were observed. A significant interaction of letter-sound congruency and visual noise was found in the left STS extending into the MTG (Fig. 4A; $p<0.05$ ). Post hoc ROI GLM analysis indicated that this interaction reflected a congruency effect in the low visual noise condition $\left(t_{18}=2.6, p<0.05\right)$ and that this effect was not significant for the medium- and high-noise levels (Fig. 4B). To further explore the nature of the significant congruency effect in the low-noise condition, direct comparisons of STS/MTG responses to congruent and incongruent letter-sound pairs at low visual noise vs. the unisensory auditory condition were performed. A significant reduction in activity for the incongruent vs. the auditory condition was revealed $\left(t_{18}=-2.56, p\right.$ $<0.05)$. 

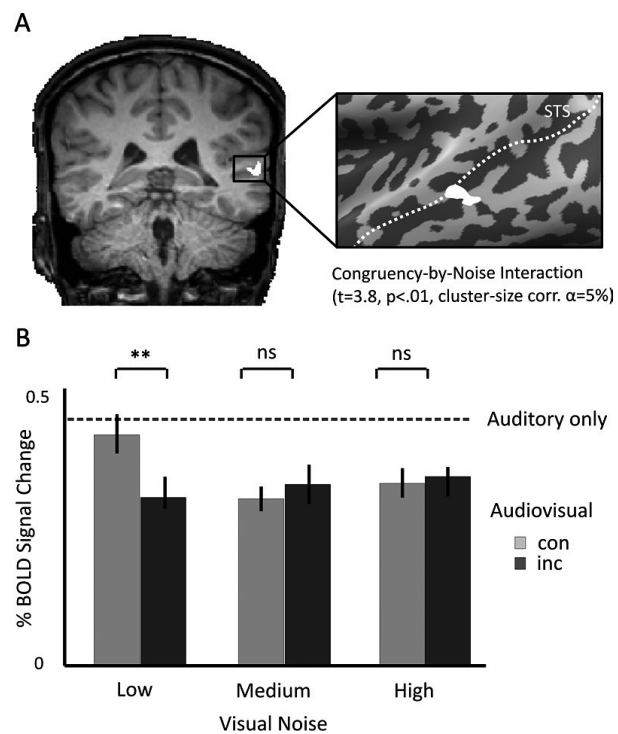

Figure 4. Group results for congruency-by-noise interaction analysis (GLM2) corrected for cluster size (alpha $<0.05$ ), projected on anatomical scan (left) and inflated cortical surface (right) of one representative subject $(A)$ and corresponding peak BOLD percent signal change values in left STS /MTG region of interest $(B)(* * p<0.01$, also see Table 1$)$.

Interestingly, the pattern of $\mathrm{FMRI}$ responses shown in the congruency by noise interaction resembled the behavioral interaction effect in that stronger STS/MTG signal changes corresponded to higher accuracy and speedier reaction times during low-noise audiovisual trials in the congruent condition (see behavior results). However, whereas the finding of an interaction effect on speech identification was due to parametric influences of visual noise on letter-speech sound congruency, cortical responses in STS/MTG exhibited rather stepwise instead of gradual influences of visual noise. This lack of a parametric effect on STS/MTG responses may be due to many factors, including the weakened behavioral effects during scanning and the indirect nature of the BOLD signal. 


\section{POST HOC CONGRUENCY CONTRASTS}

We further contrasted audiovisual congruent and incongruent conditions for all noise levels separately in order to explore the congruency effects per noise level on a whole brain level. This analysis is free from the constraint that activity has to vary as a function of visual noise and can therefore be considered more liberal. We found congruency effects within the low visual noise condition (AV1 con vs. AV1 inc) only, as shown in Fig. 5. Activated brain areas included a region of the anterior left STG, STS including medial MTG, and portions of the fusiform gyrus (FG) bilaterally in proximity to what has been termed the visual word form area (Cohen et al., 2002). A region in HS/PT (Fig. 4) was additionally activated. The left HS/PT ROI did not survive the cluster size criterion of 149 activated voxels; however, in agreement with previous findings, this activation is reported at a smaller cluster size ( 82 voxels). Finally, stronger activation for congruent vs. incongruent letter-speech sound combinations was found in the precentral gyri bilaterally and in the left putamen. ROI-based GLMs further indicated that activation to congruent lettersound pairs was stronger in all selected ROIs than activation related to the presentation of speech sounds in isolation [left anterior STG: $t_{18}=2.9, p<0.01$; left MTG/STS: $\mathrm{t}_{18}=3.3, \mathrm{p}<0.01 ; \mathrm{FG}: \mathrm{t}_{18}=2.7, \mathrm{p}<0.05$ (left); $\mathrm{t}_{18}=3.9, \mathrm{p}<0.01 ; \mathrm{HS} / \mathrm{PT}$ : $\mathrm{t}_{18}=4.2, \mathrm{p}<0.01$ (left); $\mathrm{t}_{18}=2.7, \mathrm{p}<0.05$ (right)]. In contrast, responses to incongruent combinations of letters and speech sounds did differ from speech sounds in isolation only in left STS/MTG and right HS/PT; in these areas the BOLD response to incongruent letter-speech sound pairs was reduced compared with speech sounds alone $\left(\mathrm{t}_{18}=5.3, \mathrm{p}<0.001 ; \mathrm{HS} / \mathrm{PT}: \mathrm{t}=2.55, \mathrm{p}<0.05\right)$. A complete listing of the results reported in the different analyses is given in Table 1.

Table 1. ROI details and statistics per analysis

\begin{tabular}{|c|c|c|c|c|c|c|c|c|c|c|}
\hline \multirow[t]{2}{*}{ Brain area } & \multirow[t]{2}{*}{ Hemisphere } & \multicolumn{3}{|c|}{$\begin{array}{l}\text { Talairach } \\
\text { coordinates }\end{array}$} & \multirow[t]{2}{*}{ Voxels (n) } & \multirow{2}{*}{$\begin{array}{c}\text { Effect size* } \\
\mathrm{t}\end{array}$} & \multicolumn{4}{|c|}{ Pairwise comparisons } \\
\hline & & $x$ & y & $\mathrm{z}$ & & & $\mathrm{p}$ & Con $>A$ & Inc $>A$ & Con>Inc \\
\hline \multicolumn{11}{|c|}{ Interaction test used for ROI selection§ } \\
\hline STS & Left & -58 & -37 & 7 & 98 & 3.23 & .00067 & 0.93 & 0.04 & 0.03 \\
\hline \multicolumn{11}{|c|}{ Congruency test used for $\mathrm{ROI}$ selection§ } \\
\hline FG & Left & -35 & -42 & -16 & 286 & 3.20 & 0.0008 & 0.03 & 0.66 & 0.00 \\
\hline FG & Right & 32 & -43 & -17 & 254 & 3.08 & 0.0066 & 0.00 & 0.64 & 0.00 \\
\hline aSTG & Left & -56 & -1 & 4 & 149 & 3.31 & 0.0041 & 0.01 & 0.06 & 0.00 \\
\hline STS/MTG & Left & -47 & -44 & 2 & 169 & 3.25 & 0.0048 & 0.01 & 0.00 & 0.00 \\
\hline $\mathrm{HS} / \mathrm{PT}$ & Left & -54 & -19 & 12 & 82 & 5.51 & 0.0012 & 0.00 & 0.10 & 0.00 \\
\hline HS/PT & Right & -50 & -15 & 13 & 147 & 4.99 & 0.0011 & 0.02 & 0.03 & 0.00 \\
\hline PCG & Left & -47 & -27 & 39 & 258 & 2.86 & 0.0123 & & & \\
\hline PCG & Right & 46 & -28 & 41 & 219 & 3.05 & 0.0009 & & & \\
\hline Putamen & Left & -22 & 17 & 8 & 165 & 2.48 & 0.0131 & & & \\
\hline
\end{tabular}

FG, fusiform gyrus; HS/PT, Heschl sulcus/planum temporale; MTG, middle temporal gyrus; PCG, precentral gyrus; STS, superior temporal sulcus. *Average t-value and $\mathrm{p}$-value for all voxels within a ROI; $\S$ Statistical tests used for ROI selection (corrected for cluster size at alpha $=5 \%$ ). 

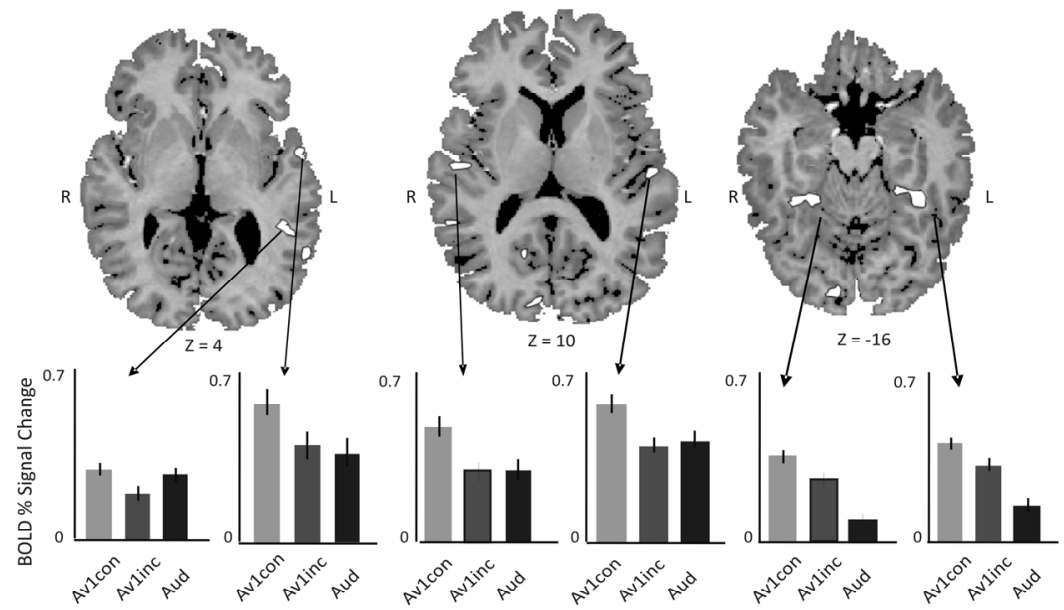

B Left ant. Superior Temporal Gyrus Left Heschl Gyrus/ Planum Temp. Left Fusiform Gyrus
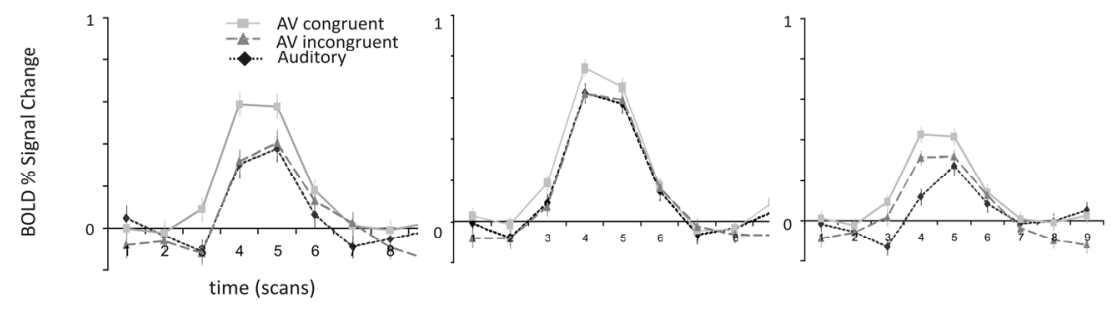

Figure 5. Group results for the congruency contrast (GLM1: AV1con > AV1inc). (A) Loci of activation are shown for three regions of interest: left anterior superior temporal gyrus (STG) and left STS/middle temporal gyrus (MTG) (left), left and right HS/PT (middle), and FG bilaterally (right). The corresponding peak percent signal change values are shown for audiovisual congruent (light gray bars), incongruent (dark gray bars) and auditory (stripes) conditions (Table 1). (B) Example BOLD response time courses for three regions of interest in the left hemisphere (also see Table 1).

\section{DISCUSSION}

The first goal of the present study was to investigate the influence of taskirrelevant visual letters on the unisensory identification of speech sounds. We found visual influences on speech processing in several brain regions, including the superior temporal cortex, auditory cortex and extrastriate visual cortex. Secondly, we investigated the behavioral relevance of the neural integration of letters and speech sounds for speech identification. In order to address this question, we manipulated the quality of the visual letter parametrically. Our results demonstrate similar effects of visual stimulus quality on the neural processing of letter-speech sound pairs in STS/MTG and on the behavioral identification of speech. 


\section{BRAIN REGIONS SUPPORTING THE VISUAL INFLUENCE ON SPEECH SOUND PROCESSING}

Cortical responses in a network of brain regions including STS, as well as 'modalityspecific' auditory (HS/PT; aSTG) and visual (FG) association cortex were influenced by the co-presentation of visual letters during speech identification. In the absence of any explicit requirement to process the letters, those regions responded more vigorously to congruent as opposed to incongruent bimodal stimulation. Analogously, as indicated by accuracy and reaction time data, speech sound identification was facilitated in the context of congruent letters and inhibited in the context of incongruent letters. In line with these data, previous behavioral studies found that the presentation of congruent syllable primes facilitated the detection of a multisensory target (Dijkstra et al., 1993; Dijkstra et al., 1989).

STS is a well-known candidate structure for the integration of audiovisual (and tactile) information as it receives input from multiple senses via cortical and subcortical connections (Beauchamp et al., 2004; Macaluso et al., 2004). Our finding of a modulation of STS responses to speech sounds by letters indicates multisensory visual influences in this region, consistent with previous reports on passive letterspeech sound integration (van Atteveldt et al., 2004; van Atteveldt et al., 2007a) and the integration of multisensory speech during active and passive tasks (Calvert et al., 2000; Olson et al., 2002; Raij et al., 2000; Wright et al., 2003). Moreover, the location of the present STS result closely matched that of previous neuroimaging investigations on letters and speech sounds (van Atteveldt et al., 2004; van Atteveldt et al., 2007a) and audiovisual speech (Beauchamp et al., 2004; Sekiyama et al., 2003). Interestingly, the congruency effect in STS was mainly characterized by a stronger suppression of incongruent than congruent letters-speech sound pairs compared with speech sounds in isolation. One potential explanation for this finding might be that the fairly strong responses to unisensory auditory stimulation in the present tasks increased the likelihood for finding multisensory suppression at the cost of finding multisensory enhancement effects. Monkey electrophysiological work has shown that a strong unisensory responsiveness of neurons allows for relatively less enhancement of cortical firing than a weak unisensory response (Stanford et al., 2005). In the present study, this auditory saturation might be explained by the unisensory auditory task with corresponding allocation of attention to the auditory modality. More importantly, however, the effect of congruency on STS responses was elicited by task-irrelevant visual letters, indicating the automatic multisensory modulation of STS responses to speech sounds. This finding indicates the existence of strong, automatic mappings between orthography and phonology in literate adults.

Multisensory visual influences on speech processing were furthermore observed in regions of the posterior (HS/PT; see Fig. 5) and anterior (aSTG; see Fig. 5) auditory association cortex. HS/PT activation is generally associated with auditory, prephonemic analysis of complex sounds (Binder et al., 1996; Seifritz et al., 2002), but 
has also been found for the integration of spoken and written language (Nakada et al., 2001; van Atteveldt et al., 2004) and the acquisition of new audiovisual associations (Hasegawa et al., 2004; Hashimoto and Sakai, 2004). Before learning to read, letters and speech sounds represent new, artificial audiovisual associations that have to be explicitly learned. PT might provide the necessary link between sensory representations of letters and speech sounds with motor representations involved in reading/speaking (Wilson et al., 2004) and writing (Longcamp et al., 2003). Anterior temporal lobe responses, in comparison, are more closely associated with the processing of intelligible than non-intelligible speech (Hwang et al., 2006; Scott et al., 2000; Scott and Johnsrude, 2003), and have even been explicitly linked to the processing and categorization of vowels (Obleser et al., 2006). Given that information about the identity of the speech sound is presumably already available in aSTG, congruency effects of visual letters on the processing of speech sounds might be expected in this region. This interpretation is in accordance with neurofunctional models of speech perception, which have implicated anterior parts of STS and STG as being part of a 'what' pathway for multisensory object recognition (Sestieri et al., 2006). In contrast, posterior auditory brain areas, such as PT, have been implicated in integrating sensory with motor representations of speech (Buchsbaum et al., 2005; Scott, 2005) and might therefore be involved in a 'how' pathway for audiovisual processing. It is interesting to consider the anatomical localization of posterior and anterior STS in this respect. Whereas posterior STS is anatomically closer to the dorsal processing pathways, anterior STS is closer to the ventral pathways (Sestieri et al., 2006). Although speculative at this point, this observation underpins the existence of a neurofunctional distinction into a 'what' stream for multisensory identity matching and a 'how' stream for matching sensory with motor representations.

A neural mechanism that can be put forward to account for the modulation of activity in STS and HS/PT by congruency is the neural integration of letters and speech sounds (van Atteveldt et al., 2004). Congruent as opposed to incongruent letter-sound pairs represent overlearned audiovisual associations for which a strong neural association exists (Froyen et al., 2008). Information about the identity of unisensory auditory and visual input has to be integrated in order to differentiate matching from non-matching letter-speech sound pairs and, hence, neural responses in STS and auditory cortex most likely reflect audiovisual integration. Interestingly, as letters and speech sounds are not naturally linked before learning to read and their mapping has to be established through intensive training, the question rises which neural mechanisms support the perceptional shift from unfamiliar to familiar audiovisual objects for congruent letter-speech sound pairs. Hein and coworkers (2007) have investigated the relative influence of audiovisual object familiarity and congruency in recruiting cortical areas involved in audiovisual integration. Comparable to the present effects of congruency under low noise in STS and ASTG, the authors found posterior STS and STG to respond only for highly familiar and semantically congruent audiovisual stimuli. Other brain regions, such 
as inferior frontal cortex, in contrast responded to the learning of new audiovisual associations (Hein et al., 2007). Similarly to the functional specialization for familiar objects that has been proposed for the visual system (Gauthier et al., 1999), familiarity or frequency of exposure might guide the functional specialization of neural systems for multisensory integration.

Finally, increased activation for congruent vs. incongruent letter-speech sound pairs was found in the FG (see Fig. 5), in close proximity to areas specialized for the processing of letter strings (Cohen and Dehaene, 2004; Cohen et al., 2002; Flowers et al., 2004; McCandliss et al., 2003) or words (Kronbichler et al., 2004). Modulation of FG by congruency has been previously reported in studies using audiovisual speech (Bushara et al., 2003; Calvert et al., 2000; Macaluso et al., 2004). Interesting in this respect is the finding that the visual processing region typically described as FG might contain a lateral subregion, the lateral inferior multisensory area that essentially possesses multisensory response properties (Cohen et al., 2004). Thus far, neurofunctional models on the integration of letters and speech sounds do not incorporate a role for visual cortical regions in letter-sound integration (van Atteveldt et al., 2004). The current findings, however, indicate that under certain task conditions effects of congruency are to be observed not only in the auditory cortex but also in the extrastriate visual cortex.

\section{BEHAVIORAL RELEVANCE OF LETTER-SPEECH SOUND INTEGRATION}

The second main finding of the present study was that neural responses in the superior temporal cortex were similarly influenced by letter-speech sound congruency and visual noise as were behavioral responses to speech sounds. That is, the speed and accuracy of speech sound identification critically depended on the quality of the visual stimulus (congruency-by-noise interaction) where facilitation/inhibition effects were reduced as a function of letter quality. This finding suggests two things: first, it indicates that in STS/MTG basic stimulus features and stimulus content interact to mediate the multisensory visual influences on speech identification, whereas earlier processing regions (HS/PT, FG) are influenced only by stimulus content. Previous findings have demonstrated a sensitivity of the left STS also for other binding factors such as temporal synchrony (Bushara et al., 2003; Calvert et al., 2000; Macaluso et al., 2004) and various linguistic and non-linguistic audiovisual inputs (Beauchamp et al., 2004; Calvert et al., 2000), supporting a general integrative function for STS. Moreover, non-human electrophysiological work has indicated that STS, unlike PT for example, possesses an audiovisual response profile with convergent inputs from visual auditory and somatosensory cortex, which makes STS a likely candidate for early integration of AV inputs (Schroeder and Foxe, 2002). Second and more importantly, demonstrating analogous effects of visual noise on neural responses to speech sounds in STS/MTG and on the behavioral identification of speech sounds provides evidence for a role of STS/MTG in the multisensory facilitation of speech perception. The present data 
therefore provide a critical extension to previous studies on audiovisual integration using either passive perception tasks (Calvert et al., 2000; Olson et al., 2002; van Atteveldt et al., 2004; van Atteveldt et al., 2007a) or indirect performance measures (Callan et al., 2003; Macaluso et al., 2004).

\section{SUMMARY AND CONCLUSION}

We have described the results of a two-experiment behavioral and fMRI study, in which the influences of visual letters on speech sound identification were investigated. Our finding that letter-speech sound congruency modulated cortical responses to speech sounds in heteromodal superior temporal cortex as well as auditory and visual association cortex indicates automatic influences of visual letters on speech sound processing. More generally, these data support the existence of a strong mapping between orthography and phonology in literate adults. The finding that adding visual noise to the letter stimulus led to a reduction of an effect of congruency, with remarkably similar neurofunctional (in STS/MTG) and behavioral response patterns (congruency-by-noise interaction), provides evidence for a functional involvement of heteromodal superior temporal cortex in the facilitation/inhibition of speech identification. The present findings thus substantiate the hypothesis of an automatic neural mechanism for the integration of letter and speech sounds in literate adults that might be critical for associating spoken and written language. The acquisition and automatization of letter- speech sound associations is an important milestone in learning to read (Ehri, 2005). Failure to acquire sufficiently automatized letter-speech sound associations has been linked to dyslexia (Vellutino et al., 2004). Therefore, knowledge on the automaticity and behavioral relevance of letter-speech sound integration in skilled readers adds to a basic framework for investigating the neural underpinnings of normal and abnormal reading development.

Acknowledgement: This research was partly supported by the Dutch Health Care Insurance Board (CVZ 608/001/2005) to L.B. 


\section{REFERENCES}

Beauchamp MS, Argall BD, Bodurka J, Duyn JH, Martin A. Unraveling multisensory integration: patchy organization within human STS multisensory cortex. Nat Neurosci 2004; 7: 1190-2.

Binder JR, Frost JA, Hammeke TA, Rao SM, Cox RW. Function of the left planum temporale in auditory and linguistic processing. Brain 1996; 119 ( Pt 4): 1239-47.

Booth JR, Burman DD, Meyer JR, Gitelman DR, Parrish TB, Mesulam MM. Functional anatomy of intra- and multisensory lexical tasks. Neuroimage 2002; 16: 7-22.

Booth JR, Cho S, Burman DD, Bitan T. Neural correlates of mapping from phonology to orthography in children performing an auditory spelling task. Dev Sci 2007; 10: 441-51.

Borowsky R, Owen WJ, Fonos N. Reading speech and hearing print: constraining models of visual word recognition by exploring connections with speech perception. Can J Exp Psychol 1999; 53: 294-305.

Buchsbaum BR, Olsen RK, Koch PF, Kohn P, Kippenhan JS, Berman KF. Reading, hearing, and the planum temporale. Neuroimage 2005; 24: 444-54.

Bushara KO, Hanakawa T, Immisch I, Toma K, Kansaku K, Hallett M. Neural correlates of multisensory binding. Nat Neurosci 2003; 6: 190-5.

Busse L, Roberts KC, Crist RE, Weissman DH, Woldorff MG. The spread of attention across modalities and space in a multisensory object. Proc Natl Acad Sci U S A 2005; 102: 18751-6.

Callan DE, Jones JA, Munhall K, Callan AM, Kroos C, Vatikiotis-Bateson E. Neural processes underlying perceptual enhancement by visual speech gestures. Neuroreport 2003; 14: 2213-8.

Calvert GA, Campbell R, Brammer MJ. Evidence from functional magnetic resonance imaging of multisensory binding in the human heteromodal cortex. Curr Biol 2000; 10: 649-57.

Cohen L, Dehaene S. Specialization within the ventral stream: the case for the visual word form area. Neuroimage 2004; 22: 466-76.

Cohen L, Jobert A, Le Bihan D, Dehaene S. Distinct unisensory and multimodal regions for word processing in the left temporal cortex. Neuroimage 2004; 23: 1256-70.

Cohen L, Lehericy S, Chochon F, Lemer C, Rivaud S, Dehaene S. Language-specific tuning of visual cortex? Functional properties of the Visual Word Form Area. Brain 2002; 125: 1054-69.

Dijkstra T, Frauenfelder UH, Schreuder R. Bidirectional grapheme-phoneme activation in a bimodal detection task. J Exp Psychol Hum Percept Perform 1993; 19: 931-50.

Dijkstra T, Schreuder R, Frauenfelder UH. Grapheme context effects on phonemic processing. Language and Speech 1989; 32: 98-108.

Ehri LC. Development of sight word reading:phases and findings. In: Snowling MJ and Hulme C, editors. The science of reading: a handbook. Oxford: Blackwell Publishing, 2005: 135-45.

Fiebach CJ, Friederici AD, Muller K, von Cramon DY. fMRI evidence for dual routes to the mental lexicon in visual word recognition. J Cogn Neurosci 2002; 14: 11-23.

Fiez JA, Balota DA, Raichle ME, Petersen SE. Effects of lexicality, frequency, and spelling-to-sound consistency on the functional anatomy of reading. Neuron 1999; 24: 205-18.

Flowers DL, Jones K, Noble K, VanMeter J, Zeffiro TA, Wood FB, et al. Attention to single letters activates left extrastriate cortex. Neuroimage 2004; 21: 829-39.

Frost R, Katz L. Orthographic depth and the interaction of visual and auditory processing in word recognition. Mem Cognit 1989; 17: 302-10.

Froyen D, Van Atteveldt N, Bonte M, Blomert L. Multisensory enhancement of the MMN to speech-sounds indicates early and automatic integration of letters and speech-sounds. Neurosci Lett 2008; 430: 23-8.

Gauthier I, Tarr MJ, Anderson AW, Skudlarski P, Gore JC. Activation of the middle fusiform 'face area' increases with expertise in recognizing novel objects. Nat Neurosci 1999; 2: 568-73. 
Goebel R, Esposito F, Formisano E. Analysis of functional image analysis contest (FIAC) data with brainvoyager QX: From single-subject to cortically aligned group general linear model analysis and self-organizing group independent component analysis. Hum Brain Mapp 2006; 27 : 392-401.

Hasegawa T, Matsuki K, Ueno T, Maeda Y, Matsue Y, Konishi Y, et al. Learned audio-visual multisensory associations in observed piano playing activate the left planum temporale. An fMRI study. Brain Res Cogn Brain Res 2004; 20: 510-8.

Hashimoto R, Sakai KL. Learning letters in adulthood: direct visualization of cortical plasticity for forming a new link between orthography and phonology. Neuron 2004; 42: 311-22.

Hein G, Doehrmann O, Muller NG, Kaiser J, Muckli L, Naumer MJ. Object familiarity and semantic congruency modulate responses in cortical audiovisual integration areas. J Neurosci 2007; 27: 7881-7.

Hwang $\mathrm{JH}, \mathrm{Wu} \mathrm{CW}$, Chen JH, Liu TC. The effects of masking on the activation of auditoryassociated cortex during speech listening in white noise. Acta Otolaryngol 2006; 126: 916-20.

Jancke L, Wustenberg T, Scheich $\mathrm{H}$, Heinze HJ. Phonetic perception and the temporal cortex. Neuroimage 2002; 15: 733-46.

Kronbichler M, Hutzler F, Wimmer H, Mair A, Staffen W, Ladurner G. The visual word form area and the frequency with which words are encountered: evidence from a parametric $\mathrm{fMRI}$ study. Neuroimage 2004; 21: 946-53.

Longcamp M, Anton JL, Roth M, Velay JL. Visual presentation of single letters activates a premotor area involved in writing. Neuroimage 2003; 19: 1492-500.

Macaluso E, George N, Dolan R, Spence C, Driver J. Spatial and temporal factors during processing of audiovisual speech: a PET study. Neuroimage 2004; 21: 725-32.

McCandliss BD, Cohen L, Dehaene S. The visual word form area: expertise for reading in the fusiform gyrus. Trends Cogn Sci 2003; 7: 293-299.

McDonald JJ, Teder-Salejarvi WA, Di Russo F, Hillyard SA. Neural substrates of perceptual enhancement by multisensory spatial attention. J Cogn Neurosci 2003; 15: 10-9.

Nakada T, Fujii Y, Yoneoka Y, Kwee IL. Planum temporale: where spoken and written language meet. Eur Neurol 2001; 46: 121-5.

Obleser J, Boecker H, Drzezga A, Haslinger B, Hennenlotter A, Roettinger M, et al. Vowel sound extraction in anterior superior temporal cortex. Hum Brain Mapp 2006; 27: 562-71.

Olson IR, Gatenby JC, Gore JC. A comparison of bound and unbound audio-visual information processing in the human cerebral cortex. Brain Res Cogn Brain Res 2002; 14: 129-38.

Perre L, Ziegler JC. On-line activation of orthography in spoken word recognition. Brain Res 2008; 1188: $132-8$.

Proverbio AM, Vecchi L, Zani A. From orthography to phonetics: ERP measures of grapheme-tophoneme conversion mechanisms in reading. J Cogn Neurosci 2004; 16: 301-17.

Raij T, Uutela K, Hari R. Audiovisual integration of letters in the human brain. Neuron 2000; 28 : 617-25.

Schroeder CE, Foxe JJ. The timing and laminar profile of converging inputs to multisensory areas of the macaque neocortex. Brain Res Cogn Brain Res 2002; 14: 187-98.

Scott SK. Auditory processing--speech, space and auditory objects. Curr Opin Neurobiol 2005; 15 : 197-201.

Scott SK, Blank CC, Rosen S, Wise RJ. Identification of a pathway for intelligible speech in the left temporal lobe. Brain 2000; 123 Pt 12: 2400-6.

Scott SK, Johnsrude IS. The neuroanatomical and functional organization of speech perception. Trends Neurosci 2003; 26: 100-7.

Seifritz E, Neuhoff JG, Bilecen D, Scheffler K, Mustovic H, Schachinger H, et al. Neural processing of auditory looming in the human brain. Curr Biol 2002; 12: 2147-51. 
Sekiyama K, Kanno I, Miura S, Sugita Y. Auditory-visual speech perception examined by fMRI and PET. Neurosci Res 2003; 47: 277-87.

Sestieri C, Di Matteo R, Ferretti A, Del Gratta C, Caulo M, Tartaro A, et al. "What" versus "where" in the audiovisual domain: an fMRI study. Neuroimage 2006; 33: 672-80.

Simos PG, Fletcher JM, Bergman E, Breier JI, Foorman BR, Castillo EM, et al. Dyslexia-specific brain activation profile becomes normal following successful remedial training. Neurology 2002; 58: 1203-13.

Stanford TR, Quessy S, Stein BE. Evaluating the operations underlying multisensory integration in the cat superior colliculus. J Neurosci 2005; 25: 6499-508.

Tagamets MA, Novick JM, Chalmers ML, Friedman RB. A parametric approach to orthographic processing in the brain: an fMRI study. J Cogn Neurosci 2000; 12: 281-97.

Talairach J, Tournoux P. Co-Planar Stereotactic Atlas of the Human Brain. Stuttgart: Thieme, 1988.

van Atteveldt N, Formisano E, Goebel R, Blomert L. Integration of letters and speech sounds in the human brain. Neuron 2004; 43: 271-82.

van Atteveldt NM, Formisano E, Blomert L, Goebel R. The effect of temporal asynchrony on the multisensory integration of letters and speech sounds. Cereb Cortex 2007; 17: 962-74.

Vellutino FR, Fletcher JM, Snowling MJ, Scanlon DM. Specific reading disability (dyslexia): what have we learned in the past four decades? J Child Psychol Psychiatry 2004; 45: 2-40.

Wilson SM, Saygin AP, Sereno MI, lacoboni M. Listening to speech activates motor areas involved in speech production. Nature Neuroscience 2004; 7: 701-702.

Wright TM, Pelphrey KA, Allison T, McKeown MJ, McCarthy G. Polysensory interactions along lateral temporal regions evoked by audiovisual speech. Cereb Cortex 2003; 13: 1034-43. 

REDUCED NEURAL INTEGRATION OF LETTERS AND SPEECH SOUNDS LINKS PHONOLOGICAL AND READING DEFICITS IN ADULT DYSLEXIA

Based on:

Blau, V., van Atteveldt, N., Ekkebus, M., Goebel, R., Blomert, L. (2009). Reduced neural integration of letters and speech sound links phonological and reading deficits in adult dyslexia. Current Biology, 19, 503-508. 


\begin{abstract}
Developmental dyslexia is a specific reading and spelling deficit affecting 4 to $10 \%$ of the population. Advances in understanding its origin support a core deficit in phonological processing characterized by difficulties in segmenting spoken words into their minimally-discernable speech segments (speech sounds/phonemes) (Shaywitz and Shaywitz, 2005; Vellutino et al., 2004) and underactivation of left superior temporal cortex. A suggested but unproven hypothesis is that this phonological deficit impairs the ability to map speech sounds onto their homologous visual letters, which in turn prevents the attainment of fluent reading levels. The present functional magnetic resonance imaging (fMRI) study investigated the neural processing of letters and speech sounds in unisensory (visual, auditory) and multisensory (audiovisual congruent, audiovisual incongruent) conditions as a function of reading ability. Our data reveal that adult dyslexic readers underactivate superior temporal cortex for the integration of letters and speech sounds. This reduced audiovisual integration is directly associated with a more fundamental deficit in auditory processing of speech sounds, which in turn predicts performance on phonological tasks. The data provide a neurofunctional account of developmental dyslexia, in which phonological processing deficits are linked to reading failure through a deficit in neural letter-speech sound integration.
\end{abstract}




\section{INTRODUCTION AND RESULTS}

Successful acquisition of basic letter-speech sound (LS) mappings is crucial for the ability to attain fluent reading skills (Ehri, 2005b). Functional magnetic resonance imaging ( $\mathrm{fMRI}$ ) in non-impaired readers has identified heteromodal superior temporal sulcus/gyrus (STS/STG) and auditory cortex (Heschl Sulcus/HS, Planum Temporale/PT) as integration sites for letters and speech sounds (LS) (Hashimoto and Sakai, 2004; van Atteveldt et al., 2004). Reading problems in dyslexia have been primarily associated with a deficit in adequately representing the smallest speech segments (speech sounds/phonemes) (Shaywitz and Shaywitz, 2005; Vellutino et al., 2004), which in turn has been suggested to interfere with the acquisition of LS mappings and hence with the progression from letter-by-letter to fluent automated reading (Vellutino et al., 2004). The present $\mathrm{fMRI}$ study examined the neurofunctional correlates of LS integration as a function of reading ability. 13 non-impaired readers and 13 dyslexic readers matched for educational level, age, handedness and IQ (Wechsler Intelligence Scale for Adults, standard scores non-impaired $=11.15$, dyslexic $=10.42$ ) were tested on a battery of measures for reading status. All dyslexic readers showed impaired reading (within the lower $10^{\text {th }}$ percentile rank on a standardized test) and poor performance on subtest involving word reading, phonological awareness, phonological decoding, and spelling (see Supplementary Materials). Letters and speech sounds were presented during scanning in four experimental conditions: visual, auditory, audiovisual congruent, audiovisual incongruent.

The first step in the fMRI analysis was to assess the relative contribution of unisensory auditory and visual responses against baseline using a multisubject general linear model (GLM 1) in each reading group. Figure 1 demonstrates that dyslexic and non-impaired activated a comparable network of brain regions in response to unisensory presented letters (occipito-temporal cortex and inferior-parietal lobule shown in green) and unisensory speech sounds (HS/PT, STG shown in red). Furthermore, cortical sites that were activated for both unisensory stimuli in fluent and dyslexic readers were found in the lower bank of STG and STS, structures previously implicated in LS convergence and integration (van Atteveldt et al., 2004). A second step was to examine potential group differences in LS processing between dyslexic and fluent readers by computing the interaction between 'reading status' and 'experimental condition' using a mixed $2 \times 4$ factorial model (GLM2). No main effect of reading status was found, but the interaction with condition revealed a bilateral cluster on STG anterior-lateral to primary auditory cortex $\left(F_{3,72}=\right.$ $14.3, p=.000$ left, $F_{3,72}=7.3, p=.000$ right; Figure $2 A$; Talairach coordinates: $x=-46$, $y=-26, z=6$ (left), $x=45, y=-22, z=7$ (right)). Here, the BOLD responses in the dyslexic group was reduced for unisensory presentations of speech sounds $\left(t_{24}=4.99, p=\right.$ .000 left, $t_{24}=3.79, p=.001$ right $)$ and congruent letter-speech sound pairs $\left(t_{24}=\right.$ $3.85, p=.001$ left, $t_{24}=2.59, p=.016$ right) (Figure $2 B$ ). Whilst these differential effects seemed slightly lateralized to the left hemisphere, the statistical interaction with hemisphere in STG did not reach significance. 

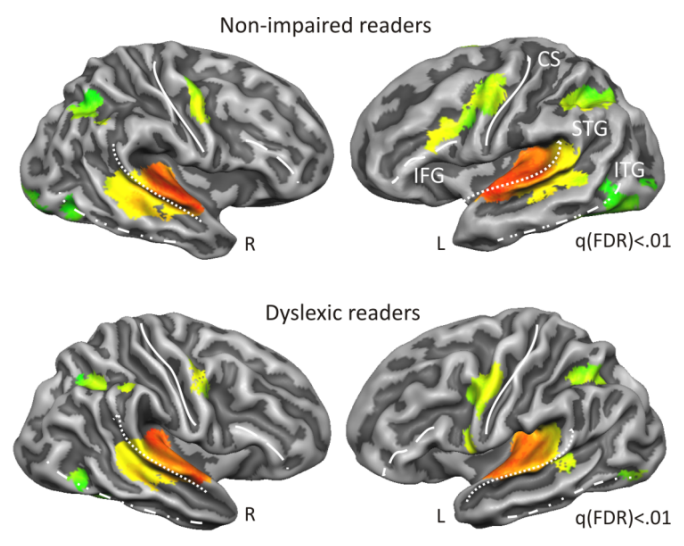

Auditory |||||||||||||||||||| visual

Figure 1. Response pattern for regions processing speech sounds (red), letters (green) or both unisensory conditions (yellow) in non-impaired (upper panel) and dyslexic readers (lower panel).

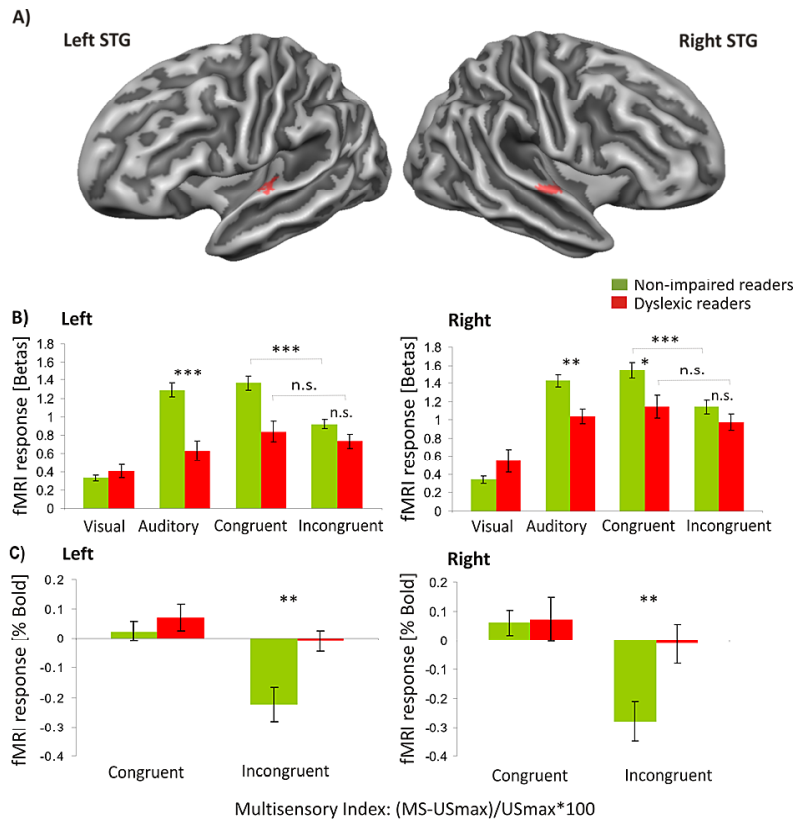

Figure 2. Group results for the 'reading status-by-condition' interaction analysis (corrected for clustersize at alpha $=.05$ ) projected on inflated cortex-based aligned group map showing clusters in bilateral STG (A). Mean BOLD response and Standard Error of Mean (SEM) for both reading groups indicates a reduced response to speech sounds and congruent LS pairs in dyslexia (B) and a reduced suppression of incongruent LS pairs relative to the maximal unisensory response (USmax) (C).

To assess whether the activation differences in STG reflect discrepancies in multisensory LS integration between dyslexic and non-impaired readers, two complementary statistical criteria were used. The congruency criterion, which indexes 
integration through stronger responses to congruent than to incongruent LS pairs (represented as AV congruent $>$ AV incongruent) directly evaluated the processing of the learned audiovisual relatedness between letters and speech sounds. Consistent with previous results [Van Atteveldt et al., 2004], we found that non-impaired readers showed stronger activation for congruent than incongruent letter-speech sound pairs in bilateral STG $\left(\mathrm{t}_{12}=5.53, \mathrm{p}=.000\right.$ left, $\mathrm{t}_{12}=6.72, \mathrm{p}=.000$ right $)$. In contrast, dyslexic readers showed no such activation difference indicating that LS integration is reduced in dyslexia. Importantly, this effect could not be explained by dyslexic readers' insufficient knowledge about LS associations, because they were highly accurate in judging the congruency of LS pairs in offline-behavioural tasks (see Supplementary Materials). Yet, dyslexic readers performed significantly slower compared to non-impaired readers indicating less automatic processing of LS mappings (Blomert and Vaessen, 2009; Schneider and Chein, 2003). The second criterion used to determine LS integration in STG was the multisensory interaction index (MSI) (Wallace et al., 1996). The MSI represents the multisensory response (MS) relative to the maximally unisensory response (USmax), where positive MSI values indicate response enhancement and negative values indicate response suppression $\left(\mathrm{MSI}=([\mathrm{MS}-\mathrm{USmax}] / \mathrm{USmax})^{* 100}\right)$. Using the MSI in addition to the congruency criterion is particularly useful in the present study where dyslexic readers show reduced activation for unisensory presentations of speech sounds, because it accounts for individual differences in unisensory response strength when classifying an area as integration site. We found that non-impaired readers exhibited response suppression in bilateral STG for incongruent LS pairs compared to the maximal unisensory response $\left(t_{12}=-3.92, p=.002\right.$ left, $t_{12}=-4.09, p=.002$ right), whereas dyslexic readers failed to show such a suppression effect (Figure 2C) (non-impaired vs. dyslexic readers: $t_{24}=-3.19, p=.004$ left, $t_{24}=-2.75, p=.011$ right). In contrast, dyslexic and non-impaired readers showed a comparable weak enhancement for congruent LS pairs. In other words, dyslexic readers failed to modulate the response to speech sounds when those sounds were presented together with both congruent and incongruent visual letters, indicating the weak or absent integration of LS in line with the previous (congruency) analysis.

The absence of multisensory enhancement effects for congruent LS pairs relative to speech sounds might be due to response saturation for processing speech sounds in unisensory conditions, similar to reports from monkey electrophysiology (Stanford et al., 2005). Although an earlier study reported multisensory enhancement effects for congruent LS pairs in non-impaired readers (van Atteveldt et al., 2004), the absence of such an effect in the present study is likely to be a consequence of the different focus in the analysis approach. Importantly, the congruency effect as well as the demonstrated suppression effect for incongruent LS pairs are in line with those previous finding, supporting the same overall conclusion that STG is involved in LS integration. 


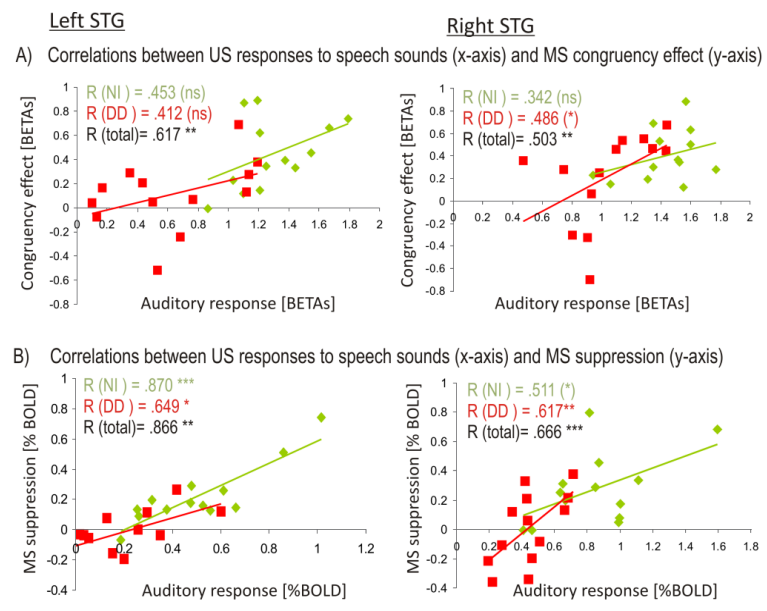

Figure 3. Pearson's correlation coefficients for the unisensory auditory response to speech sounds ( $x$ axis) and the congruency effect (congruent $>$ incongruent, $y$-axis) (A), and the unisensory auditory response to speech sounds (x-axis) and the multisensory suppression effect ([MS-USmax]/USmax)*100), $y$-axis) (B). Note that while the fMRI signal in A) is expressed in z-normalized beta values, in $B$ ) it is expressed as relative change in BOLD signal in order to avoid the MSI to reach extremely high values for occasionally very low maximal unisensory responses (see methods).

As dyslexic readers exhibited reduced responses to speech sounds and reduced indices of LS integration in STG, we evaluated how these two measures are related. Unisensory responses to speech sounds correlated positively with the congruency effect in bilateral STG across reading groups (Figure 3a) and with multisensory suppression for incongruent LS pairs across and within reading groups (Figure $3 \mathrm{~b}$ ). Thus, the correlations between the response to unisensory speech sounds and LS integration was significant independent of the statistical criterion used to determine LS integration (Congruency vs. MSI). Multisensory enhancement effects showed a significant negative correlation with the speech response in the left STG in non-impaired readers ( $R=-.576, p=.039$; data not shown), indicating that saturation effects may have prevented the observation of MS enhancement effects. These findings indicate a linear dependence between multisensory integration effects and unisensory responses to speech. The relevance of speech sound processing in STG for perception/behaviour was indicated by significant correlations with speed and accuracy of phoneme deletion within the dyslexic group (speed only: $R=-.757, p=.003$ ) and across reading groups $(R=-.651, p=.000)$ but not within the non-impaired reading group (see Supplementary Materials). As phoneme deletion is a major diagnostic index of reading problems in dyslexia, this brain-behaviour correlation suggest that STG responses to speech sounds are linked to the severity of phonological awareness deficits in dyslexic individuals. 


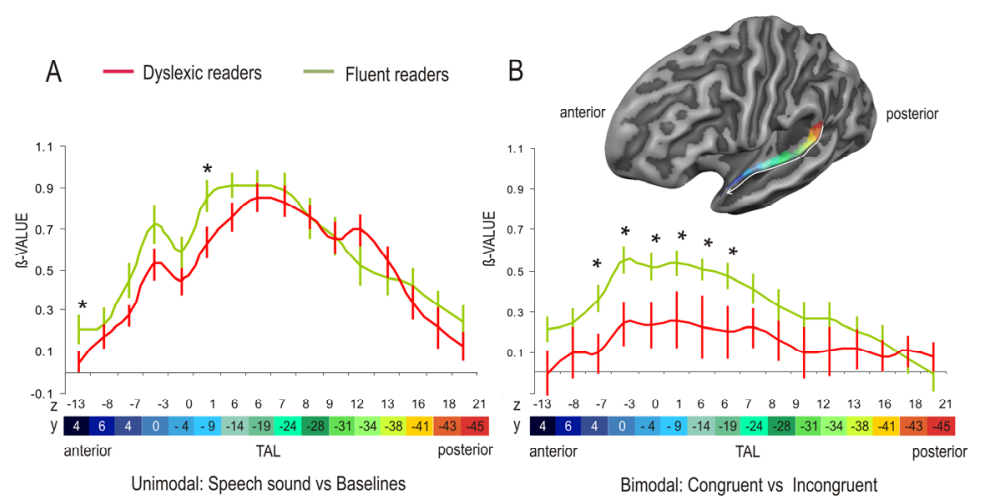

Figure 4. Mean BOLD response (Beta value) and SEM to speech sounds vs. baseline (A) and congruent vs. incongruent LS pairs (B) as a function of position on left STG from posterior (red) to anterior (blue) (lines: dyslexic readers = red; non-impaired readers = green).

To further specify the group effects for speech sounds and LS congruency beyond the region of interest revealed in the interaction analysis, we introduced STG position as a variable in the group analysis. This analysis was motivated by models of auditory processing that imply regions anterior-lateral to primary auditory cortex to be gradually more sensitive to speech than to non-speech sounds (from posterior to anterior) (Scott, 2005). Cortical responses to speech sounds vs. baseline showed a main effect of STG position $\left(F_{1,15}=16.23, p=.000\right)$ (Figure $\left.4 A\right)$, best described as a quadratic trend in both reading groups (Non-impaired: $F_{1,12}=52.45$, $p=.000$; Dyslexic: $F_{1,12}=108.73, p=.000$ ). The cluster located the middle of the sampled distribution approximately corresponded to the superior temporal region revealed by the group-by-condition interaction analysis (Figure $2 \mathrm{~A}$ ). The congruency effect showed a main effect of STG position against baseline $\left(F_{1,15}=3.556, p=\right.$ $.024)$ and a tendency for a significant interaction of reading group and STG position $\left(F_{2,15}=1.57, p=.08\right)$ (Figure $\left.4 \mathrm{~B}\right)$. Non-impaired readers showed an increased congruency effect at anterior STG sites that decreased towards posterior sites (quadratic trend, $F_{1,12}=29.18, p=.000$ ), which was not found in the dyslexic group. Group differences for the congruency effect were significant in a range of functional clusters on anterior STG (from $y=4: z=-7$ to $y=-19: z=6$ ) showing a weaker congruency effect in dyslexia. Overall, non-impaired and dyslexic readers show a rather localized difference in functional sensitivity for speech sounds, but differed to a greater extend in functional sensitivity for LS congruency.

\section{DISCUSSION}

The present fMRI study revealed that adult dyslexic readers differ from nonimpaired readers in the neural integration of basic letter-speech sound pairs within superior temporal brain regions. In line with previous neuroimaging findings in non- 
impaired readers (Blau et al., 2008; van Atteveldt et al., 2004), cortical responses to speech sounds in STG were modulated by LS congruency, an effect that was more pronounced for non-impaired than for dyslexic readers. As the congruency between letters and speech sounds cannot be established unless auditory and visual inputs have been successfully integrated, a reduced congruency effect in dyslexia is likely to reflect less successful LS integration. The gradual rise and fall of the congruency effect along the STG axis for non-impaired readers furthermore indicates the existence of a functional gradient for processing learned audiovisual associations, that is absent in dyslexia. Whether the origin of this integration deficit is entirely speech-specific (Bonte et al., 2007; Schulte-Korne et al., 1998) or has nonlinguistic roots (Birch and Belmont, 1964) remains to be determined. In addition, recent findings indicate that the LS integration deficit in dyslexia might dissociate from deficits in integrating audiovisual speech (Pekkola et al., 2006). Certainly, a failure to activate key brain structures for integrating information about letters and speech sounds is likely to interfere with the acquisition of automated LS mappings and hence with reading success. This is in line with the prediction that learning and automating LS mappings is a crucial step in literacy acquisition (Ehri, 2005b) that is impaired in dyslexia (Vellutino et al., 2004).

The present finding that dyslexic readers did not suppress STG activity to incongruent LS pairs supports and extends this conclusion. Suppressive multisensory interactions reflect the down-regulation of activity in one sensory modality by multisensory inputs and have been reported previously for audiovisual speech using fMRI (Wright et al., 2003) and event-related potentials (ERP) (van Wassenhove et al., 2005). Decreased responses to incongruent LS pairs relative to speech sounds in non-impaired readers most likely relate to their evaluation as being non-existing audiovisual pairs in their language (not associated through learning). Consequently, a failure to suppress incongruent LS pairs in dyslexia indicates the less efficient discrimination of those stimuli from existing audiovisual pairs, which might provide a way to selectively filter distracting orthographic inputs in favor of processing relevant ones. This way of prioritizing relevant information is a core feature of theories on selective attention (Desimone and Duncan, 1995) and has, within modalities, been related to the impaired filtering of behaviorally irrelevant visual information in dyslexia (Roach and Hogben, 2007). Importantly, dyslexic readers also showed strongly reduced STG responses to speech sounds suggesting a deficit in phonetic/phonological processing of speech input, which is in line with previous neuroimaging findings (Corina et al., 2001; Dufor et al., 2007; Ruff et al., 2003; Rumsey et al., 1992; Temple, 2002), although the activation focus in the present study was slightly more anterior. Moreover, cortical activity for speech sounds in dyslexic readers correlated strongly with their performance on a classical measure of phoneme awareness (phoneme deletion), which is one of the major indices of reading problems in dyslexia. This finding provides an interesting empirical link between behavioral measures of reading success and cortical processing of speech sounds in superior temporal brain regions. 
Correlations between deviant responses to speech sounds and reduced LS integration indicate that these two 'deficits' might be related, but raise questions about the nature of this relation. One possible interpretation is that a phonological processing deficit in dyslexia precedes a deficit in LS integration, which in turn causes difficulties in learning to read. This precedence of phonological deficits is supported by behavioral studies showing that phonological skills before learning to read are predictors for later reading achievements (Torgesen et al., 1994; Wagner et al., 1997). Alternatively, LS integration deficits may influence phonological processing of speech. This is supported by the finding that written language learning strongly interacts with phonological perception during development (Ziegler and Goswami, 2005) and that LS mapping tasks but not phonological tasks predict later reading deficits in preschool children genetically at-risk for dyslexia (Willems et al., submitted). Clearly, both explanations are not mutually exclusive. Future LS training and longitudinal developmental studies as well as investigations of multisensory processing of larger linguistic units are necessary to distinguish which deficit represents "cause" and "effect" and how this is reflected in the differential recruitment and connectivity of relevant brain regions.

In sum, the present data provide neurofunctional evidence for a basic letter-speech sound integration deficit in adult dyslexia as one of the proximate causes of reading and spelling failure that may bridge the gap between phonological processing deficits and problems in learning to read. 


\section{METHODS}

Thirteen dyslexic readers (mean age: 23.5, sd: 3.7, 1 female) and 13 non-impaired readers (mean age: 26.8 , sd: 5.4, 4 female) participated in the experiment (description of the dyslexic sample in supplementary methods), which was voluntary and in accordance with the Maastricht University ethical guidelines. The stimuli and presentation design were adapted from (van Atteveldt et al., 2004). Stimuli were visual letters and auditory speech sounds corresponding to Dutch single letters. Stimuli were presented using Presentation software (Neurobehavioral Systems, Inc.) in blocks corresponding to four experimental conditions: unisensory letters (L), unisensory speech sounds (S), multisensory congruent LS pairs, multisensory incongruent LS pairs. One block was 20.8 seconds long, divided into 4 mini-blocks (5.2 s). During multisensory stimulation, stimuli were presented simultaneously. Subjects passively listened to and/or viewed the stimuli. The experiment included four experimental runs, each composed of eight blocks and alternating fixation periods. Each condition (40 trials) was repeated twice per run. The order of blocks was pseudorandomized within runs and the order of runs was counterbalanced across subjects.

Blood-oxygen-level-dependent (BOLD) signals were measured using a 3 Tesla Siemens headscanner (Siemens, Erlangen, Germany). Functional MRI data were acquired using a $\mathrm{T} 2 *$-sensitive gradient echo planar imaging (EPI) sequence covering the whole-brain (24 slices, slice-thickness $4.5 \mathrm{~mm}, 3 \times 3$ in-plane resolution, repetition time $(T R)=5.2 \mathrm{~s}$, TRslice/Echo Time $(T E)=63 / 32 \mathrm{~ms}$, Field of View (FOV): $192 \mathrm{~mm}^{2}$, matrix: $\left.64 \times 64 \times 24\right)$. Volume acquisition time was $1.5 \mathrm{~s}$ followed by a silent delay of $3.7 \mathrm{~s}$ in which stimuli were presented, resulting in a TR of $5.2 \mathrm{~s}$. A high resolution T1-weighted anatomical image was acquired for each subject (MP-RAGE sequence, 176 slices, $1 \mathrm{~mm}$ slice-thickness, $1 \times 1$ in-plane resolution, $\mathrm{TR}=7.9 \mathrm{~s}, \mathrm{TE}=$ $2.4 \mathrm{~ms}$, matrix size: $256 \times 256$ ). Imaging data were analyzed using BrainVoyager $Q X$ (Brain Innovation, Maastricht, Netherlands, (Goebel et al., 2006). Functional data were preprocessed to correct for slice scan time differences, 3D motion artifacts, linear drifts, and low-frequency non-linear drifts (high pass filter $\leq 3$ cycles/time course). Functional data were then coregistered with the in-session anatomical volume and transferred into standard stereotaxic space using Talairach normalization (Talairach and Tournoux, 1988). In addition, anatomical and functional images were aligned based on individual curvature information reflecting the gyral/sulcal folding pattern (cortex-based alignment; Goebel et al., 2006), and shape-averaged folded cortical meshes were created for both hemispheres and groups. Multisubject General Linear Modal (GLM) statistics were generated by modeling the evoked hemodynamic response for all four conditions as boxcars convolved with a twogamma hemodynamic response function. Population-level inferences were based on second-level random effects contrasts with predictors separated for each subject. In a first global analysis unisensory auditory and visual conditions were contrasted against baseline (GLM 1) and a relative contribution value was calcu- 
lated in each voxel for the auditory vs. visual predictors $\left(b^{v}-b^{a}\right) /\left(b^{v}+b^{a}\right)$. In a second analysis 'reading status' (non-impaired, dyslexic) and 'stimulation condition' (L, S, LS congruent, LS incongruent; GLM 2) including interactions between the two factors were specified. Multisubject statistical maps were thresholded using the false-discovery rate (FDR, Genovese et al., 2002) for GLM 1 (Figure1\&2) and clustersize thresholding (Forman et al., 1995; Goebel et al., 2006) for GLM2, where an initial voxel-level threshold was set to $.001(t=4.25)$ uncorrected resulting in a cluster-level of $160 \mathrm{~mm}^{3}$ after 1000 iterations, corresponding to a corrected false positive probability of $5 \%$ or less (Figure3\&4). Statistical comparisons between conditions within regions of interest were based on z-normalized beta values (ynew $[\mathrm{t}]=\mathrm{y}[\mathrm{t}]-\mathrm{mean} / \mathrm{StdDev}$ ) except the computation of the multisensory interaction index (MSI: ([MS-USmax]/USmax)*100), (Meredith and Stein, 1986; Wallace et al., 1996) which was based on total, baseline-uncorrected \% signal values (Baseline $(100 \%)+$ Signal Change (e.g., $1.4 \%)=$ e.g., $101.4 \%)$ in order to avoid the MSI to reach extremely high values for occasionally very low maximal unisensory responses (van Atteveldt et al., 2007b).

Acknowledgements: This research was supported by the Dutch Health Care Insurance Board (CVZ 608/001/2005) to L.B. 


\section{REFERENCES}

Lyon, G.R., Shaywitz, S.E., and Shaywitz, B.A. (2003). A definition of Dyslexia. Annals of Dyslexia 53, 1-14

Blomert, L. (2005). Dyslexie in Nederland (Amsterdam: Uitgeverij Nieuwezijds).

Shaywitz, S.E. (1998). Dyslexia. The New England Journal of Medicine 338, 307-312.

Pennington, B.F., Van Orden, G.C., Smith, S.D., Green, P.A., and Haith, M.M. (1990). Phonological processing skills and deficits in adult dyslexics. Child Dev 61, 1753-1778.

Wagner, R., and Torgesen, J. (1987). The nature of phonological processes and its causal role in the acquisition of reading skills. Psychological Bulletin 101, 192-212.

Ramus, F. (2003). Developmental dyslexia: specific phonological deficit or general sensorimotor dysfunction? Curr Opin Neurobiol 13, 212-218.

Vellutino, F.R., Fletcher, J.M., Snowling, M.J., and Scanlon, D.M. (2004). Specific reading disability (dyslexia): what have we learned in the past four decades? J Child Psychol Psychiatry 45, 2-40.

Shaywitz, S.E., and Shaywitz, B.A. (2005). Dyslexia (specific reading disability). Biol Psychiatry 57, 1301-1309.

Rumsey, J.M., Andreason, P., Zametkin, A.J., Aquino, T., King, A.C., Hamburger, S.D., Pikus, A., Rapoport, J.L., and Cohen, R.M. (1992). Failure to activate the left temporoparietal cortex in dyslexia. An oxygen 15 positron emission tomographic study. Arch Neurol 49, 527-534.

Temple, E. (2002). Brain mechanisms in normal and dyslexic readers. Curr Opin Neurobiol 12, 178183.

Snowling, M. (2004). The Science of Dyslexia: A Review of Contemporary Approaches. In The Study of Dyslexia, Volume 1, M. Turner and J. Rack, eds. (New York: Kluwer Academic/Plenum Publishers), pp. 77-90.

Ehri, L.C. (2005). Development of sight word reading:phases and findings. In The science of reading: a handbook, M.J. Snowling and C. Hulme, eds. (Oxford: Blackwell Publishing), pp. 135-145.

Hashimoto, R., and Sakai, K.L. (2004). Learning letters in adulthood: direct visualization of cortical plasticity for forming a new link between orthography and phonology. Neuron 42, 311-322.

van Atteveldt, N., Formisano, E., Goebel, R., and Blomert, L. (2004). Integration of letters and speech sounds in the human brain. Neuron 43, 271-282.

Schneider, W., and Chein, J.M. (2003). Controlled and automatic processing: Behavior, theory and biological mechanisms. Cogntive Science 27, 525-559.

Blomert, L., and Vaessen, A. (2009). 3DM Differential Diagnostics for Dyslexia: Cognitive Analysis of Reading and Spelling (Amsterdam: Boom Test Publishers).

Wallace, M.T., Wilkinson, L.K., and Stein, B.E. (1996). Representation and integration of multiple sensory inputs in primate superior colliculus. J Neurophysiol 76, 1246-1266.

Stanford, T.R., Quessy, S., and Stein, B.E. (2005). Evaluating the operations underlying multisensory integration in the cat superior colliculus. J Neurosci 25, 6499-6508.

Scott, S.K. (2005). Auditory processing--speech, space and auditory objects. Curr Opin Neurobiol $15,197-201$.

Blau, V., van Atteveldt, N., Formisano, E., Goebel, R., and Blomert, L. (2008). Task-irrelevant visual letters interact with the processing of speech sounds in heteromodal and unisensory cortex. Eur J Neurosci 28, 500-509.

Schulte-Körne, G., Deimel, W., Bartling, J., and Remschmidt, H. (1998). Auditory processing and dyslexia: evidence for a specific speech processing deficit. Neuroreport 9, 337-340.

Bonte, M.L., Poelmans, H., and Blomert, L. (2007). Deviant neurophysiological responses to phonological regularities in speech in dyslexic children. Neuropsychologia 45, 1427-1437.

Birch, H.G., and Belmont, L. (1964). Auditory-Visual Integration in Normal and Retarded Readers. American Journal of Orthopsychiatry 34, 852-861. 
Hairston, W.D., Burdette, J.H., Flowers, D.L., Wood, F.B., and Wallace, M.T. (2005). Altered temporal profile of visual-auditory multisensory interactions in dyslexia. Exp Brain Res 166, 474-480.

Pekkola, J., Laasonen, M., Ojanen, V., Autti, T., Jaaskelainen, I.P., Kujala, T., and Sams, M. (2006). Perception of matching and conflicting audiovisual speech in dyslexic and fluent readers: an fMRI study at 3 T. Neuroimage 29, 797-807.

Wright, T.M., Pelphrey, K.A., Allison, T., McKeown, M.J., and McCarthy, G. (2003). Polysensory interactions along lateral temporal regions evoked by audiovisual speech. Cereb Cortex 13, 1034-1043.

van Wassenhove, V., Grant, K.W., and Poeppel, D. (2005). Visual speech speeds up the neural processing of auditory speech. Proc Natl Acad Sci U S A 102, 1181-1186.

Desimone, R., and Duncan, J. (1995). Neural mechanisms of selective visual attention. Annu Rev Neurosci 18, 193-222.

Roach, N.W., and Hogben, J.H. (2007). Impaired filtering of behaviourally irrelevant visual information in dyslexia. Brain 130, 771-785.

Ruff, S., Marie, N., Celsis, P., Cardebat, D., and Demonet, J.F. (2003). Neural substrates of impaired categorical perception of phonemes in adult dyslexics: an fMRI study. Brain Cogn 53, 331334.

Dufor, O., Serniclaes, W., Sprenger-Charolles, L., and Demonet, J.F. (2007). Top-down processes during auditory phoneme categorization in dyslexia: a PET study. Neuroimage 34, 1692-1707.

Corina, D.P., Richards, T.L., Serafini, S., Richards, A.L., Steury, K., Abbott, R.D., Echelard, D.R., Maravilla, K.R., and Berninger, V.W. (2001). fMRI auditory language differences between dyslexic and able reading children. Neuroreport 12, 1195-1201.

Torgesen, J.K., Wagner, R.K., and Rashotte, C.A. (1994). Longitudinal studies of phonological processing and reading. J Learn Disabil 27, 276-286; discussion 287-291.

Wagner, R.K., Torgesen, J.K., Rashotte, C.A., Hecht, S.A., Barker, T.A., Burgess, S.R., Donahue, J., and Garon, T. (1997). Changing relations between phonological processing abilities and word-level reading as children develop from beginning to skilled readers: a 5-year longitudinal study. Dev Psychol 33, 468-479.

Ziegler, J.C., and Goswami, U. (2005). Reading acquisition, developmental dyslexia, and skilled reading across languages: a psycholinguistic grain size theory. Psychol Bull 131, 3-29.

Willems, G., Poelmans, H., Richardson, U., and Blomert, L. (submitted). What preschool intervention in children at familial risk of dyslexia reveals about their core deficits and the way to treat them.

Goebel, R., Esposito, F., and Formisano, E. (2006). Analysis of functional image analysis contest (FIAC) data with brainvoyager QX: From single-subject to cortically aligned group general linear model analysis and self-organizing group independent component analysis. Hum Brain Mapp 27, 392-401.

Talairach, J., and Tournoux, P. (1988). Co-Planar Stereotactic Atlas of the Human Brain (Stuttgart: Thieme).

Genovese, C.R., Lazar, N.A., and Nichols, T. (2002). Thresholding of statistical maps in functional neuroimaging using the false discovery rate. Neuroimage $15,870-878$.

Forman, S.D., Cohen, J.D., Fitzgerald, M., Eddy, W.F., Mintun, M.A., and Noll, D.C. (1995). Improved assessment of significant activation in functional magnetic resonance imaging (fMRI): use of a cluster-size threshold. Magn Reson Med 33, 636-647.

Meredith, M.A., and Stein, B.E. (1986). Visual, auditory, and somatosensory convergence on cells in superior colliculus results in multisensory integration. J Neurophysiol 56, 640-662.

van Atteveldt, N.M., Formisano, E., Goebel, R., and Blomert, L. (2007). Top-down task effects overrule automatic multisensory responses to letter-sound pairs in auditory association cortex. Neuroimage 36, 1345-1360. 


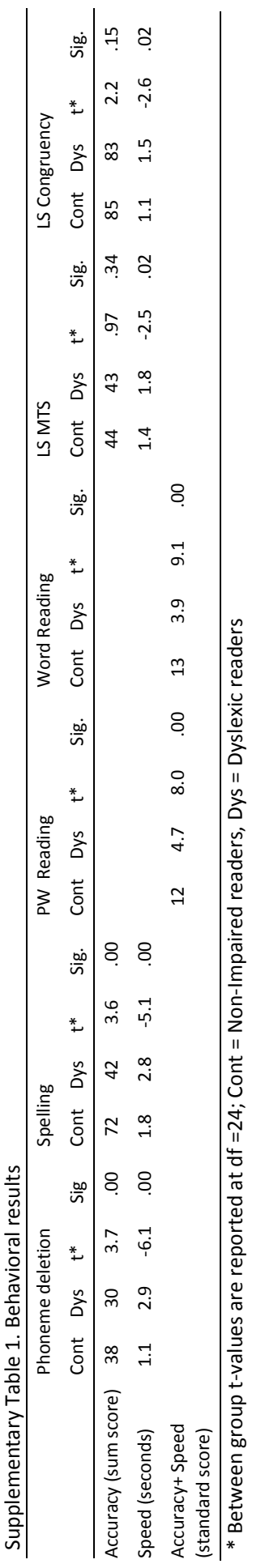


Phoneme Deletion: A classical measure of phonological awareness. In this task, a pseudoword is presented via headphones and the subject is instructed to leave out one speech sound. The task of the subject is to overtly articulate the 'new' pseudoword after the sound has been deleted. Performance independently reflects accuracy and speed.

Spelling: A measure of knowledge about spelling rules. In this test a word is presented via headphones and simultaneously a computer screen shows the parts of the same word together with four possible letters/syllables that are missing from the written word. The task of the subject is to decide as quickly as possible which of the letters/syllables correctly complement the visual words so that it matches the word presented via headphones. Performance independently reflects accuracy and speed.

Pseudoword (PW) Reading: A measure of sub-lexical reading performance. This test consists of pseudowords with different levels of difficulty listed on paper. The task of the subject is to read as many pseudowords correctly within 2 minutes. Performance on this test reflects a combination of speed and accuracy.

Word Reading: A measure of lexical reading performance. This test consists of real words with different levels of difficulty listed on paper. The task of the subject is to read as many words as possible correctly within 1 minute. Performance on this test reflects a combination of speed and accuracy.

LS Matching-to-Sample (MTS): A measure of knowledge about letter-sound pairs. A visual letter is presented simultaneously with a speech sound (not the letter name). The task of the subject is to decide as quickly as possible whether the letter and the speech sound form an existing 'pair' in Dutch (i.e. whether they represent the same letter of the alphabet) by pressing on of for buttons corresponding to four visually presented letters. Performance independently reflects accuracy and speed.

LS Congruency Judgment: A measure of knowledge about letter-sound pairs. A visual letter is presented simultaneously with either a congruent or incongruent speech sound. The task of the subjects is to decide whether the letter-speech sound 'pair' is congruent or incongruent. Performance independently reflects accuracy and speed.

Note: for more detail about the Dutch diagnostic battery see Blomert \& Vaessen (2009). 


\section{Chapter 3}

Supplementary Figure 1

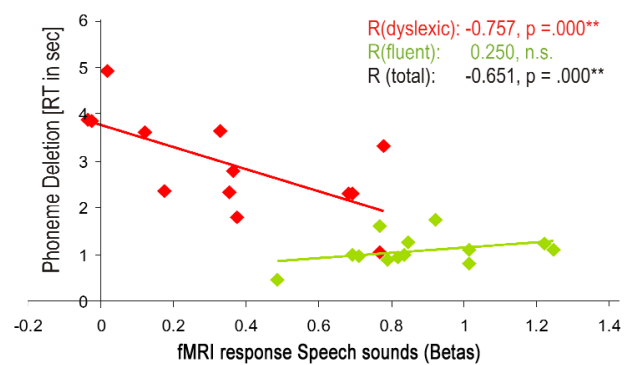

Figure 1. Correlation of the fMRI response to speech sounds with the speed on a phoneme awareness task (phoneme deletion) in fluent (green) and dyslexic readers (red)

Supplementary Figure 2 (extends Fig.1 as published)

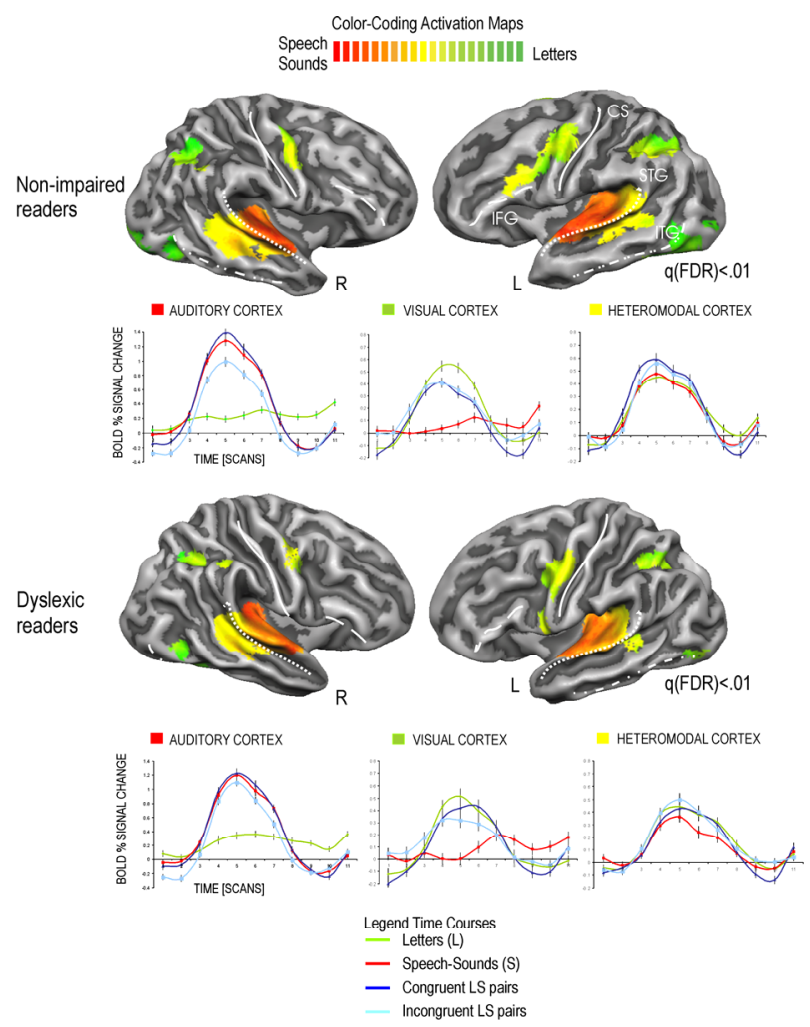

Figure 2. This full version of the figure shows the response pattern for regions processing speechs sounds (red), multisensory congruent letter-speech sound pairs (dark blue) and multisensory incongruent letter-speech sound pairs (light blue) in each of the unisensory-defined functional areas. 


\section{DEVIANT PROCESSING OF LETTERS AND SPEECH SOUNDS AS} PROXIMATE CAUSE OF READING FAILURE: A FMRI STUDY OF DYSLEXIC CHILDREN

Based on:

Blau, V., Reithler, J, van Atteveldt, N., Seitz, J., Gerretsen, P., Goebel, R., Blomert, L. Deviant processing of letters and speech sounds as proximate cause of reading failure: a $\mathrm{fMRI}$ study of dyslexic children. Brain, In revision. 


\begin{abstract}
Learning to associate auditory information of speech sounds with visual information of letters is a first and critical step for becoming a skilled reader in alphabetic languages. Nevertheless, it remains largely unknown which brain areas subserve the learning and automation of such associations. Here, we employ functional magnetic resonance imaging (fMRI) to study letter-speech sound integration in children with and without developmental dyslexia. The results demonstrate that dyslexic children show reduced neural integration of letters and speech sounds in the Planum Temporale (PT)/Heschl Sulcus (HS) and the Superior Temporal Sulcus (STS). While cortical responses to speech sounds in fluent readers were modulated by letter-speech sound congruency with strong suppression effects for incongruent letters, no such modulation was observed in the dyslexic readers. Whole-brain analyses of unisensory visual and auditory group differences additionally revealed reduced unisensory responses to letters in fusiform gyrus (FG) in dyslexic children as well as reduced activity for processing speech sounds in anterior superior temporal gyrus (aSTG), PT/HS and STS. Importantly, the neural integration of letters and speech sounds in PT/HS and the neural response to letters in FG explained almost $40 \%$ of the variance in individual reading performance. These findings indicate that an interrelated network of visual, auditory and heteromodal brain areas contributes to the skilled use of letter-speech sound associations necessary for learning to read. By extending similar findings in adults, the data furthermore argue against the notion that reduced neural integration of letters and speech sounds in dyslexia reflect the consequence of a lifetime of reading struggle. Instead, they support the view that letter-speech sound integration is an emergent property of learning to read that develops inadequately in dyslexic readers, presumably as a result of a deviant interactive specialization of neural systems for processing auditory and visual linguistic inputs.
\end{abstract}




\section{INTRODUCTION}

Learning to read is an important milestone in individual cognitive development characterized by the complex interplay of various kinds of skills and knowledge (Adams, 1994). However, the first critical step in reading development and the focus of most early reading instruction is to learn the correspondences between visual letters and auditory units of speech (speech sounds) (Ehri, 2005). Successful acquisition of letter-speech sound associations, in turn, has been theorized to be critical for the development of fluent reading skills by impacting the early phases of literacy acquisition (Bradley and Bryant, 1983; Ehri, 2005). Once letter-speech sound associations have been successfully formed they may consequently refine a child's awareness for the existence of isolated speech sounds. Thus, the relationship between reading acquisition and awareness for speech sounds is likely to be reciprocal (Ehri, 2005; Perfetti et al., 1987; Torgesen et al., 1994; Wagner and Torgesen, 1987; Ziegler and Goswami, 2005).

In largely transparent languages, such as Dutch, most school-aged children fully master letter-speech sound associations within one year of reading instruction (Blomert and Vaessen, 2009). Nonetheless, a substantial number of individuals fail to meet the standard criteria for fluent reading even after several years of schooling. Specific reading disorder (or developmental dyslexia), which affects approximately 4 to $10 \%$ of the population (Blomert, 2005; Esser et al., 1990; Shaywitz et al., 1990), is characterized by persistent difficulties in reading and/or spelling that are unexpected in relation to age, motivation or other cognitive abilities (Lyon et al., 2003). Advances in understanding the origin of dyslexia support a core deficit in phonological processing characterized by difficulties in recognizing and manipulating the sound structure of language (Share, 1995; Shaywitz and Shaywitz, 2005; Snowling, 2001; Vellutino et al., 2004). Although various other factors may play a role as well (Livingstone et al., 1991; Nicolson et al., 2001; Stein and Walsh, 1997; Tallal et al., 1993), impaired phonological awareness constitutes the most common behavioral explanation for reading failure (Ramus, 2003). Given this primacy of phonological deficits and the reciprocity between the development of reading and phonological awareness, it is surprising that the learning of letter-speech sound associations and the neural mechanisms supporting it have hardly been investigated as a function of reading ability.

In literate adults, functional Magnetic Resonance Imaging (fMRI) has been employed to reveal the involvement of superior temporal cortex (Superior Temporal Gyrus, STG/Superior Temporal Sulcus, STS) and auditory cortex (Heschl Sulcus, HS/ Planum Temporale, PT) in the integration of letters and speech sounds (Raij et al., 2000; van Atteveldt et al., 2004; van Atteveldt et al., 2007). More important, we recently tested the hypothesis that dyslexic adult readers differ from controls in letter-sound integration (Blau et al., 2009). The results showed that, relative to fluent readers, dyslexic readers underactivate STG for the integration of passively presented letter-speech sound stimuli. This reduced integration was directly asso- 
ciated with reduced auditory processing of speech sounds, which in turn predicted performance on phonological tasks. This finding suggested that the ability to efficiently integrate letters with speech sounds might indeed be one of the direct neurofunctional correlates of reading failure.

An important question that arose in response to these findings was whether the observed neural deficit in integrating letters and speech sounds resulted from a lifetime of reading difficulties or constituted a more fundamental problem instrumental in producing later reading failure. To date, however, pediatric fMRI studies have not directly investigated the associations of letters and speech sounds. Instead, complex phonological tasks have often been employed to study reading impairments in children, such as pseudoword reading or visual rhyming (Cao et al., 2006; Hoeft et al., 2007; Shaywitz et al., 2002; Temple et al., 2001). These studies converge on the finding of underactivation in perisylvian cortex and occipitotemporal gyri in dyslexia. Moreover, deviant patterns of activation in frontal cortex are reported in some but not all child fMRI studies (for review see Maisog et al., 2008). What is not clear from these studies is to what degree the rather complex phonological tasks tap into the neural mechanisms related to the processing of the most basic orthographic and phonological stimuli (letters, speech sounds) and their combinations.

Given the relevance of letter-speech sound associations for learning to read and reading failure, the main goal of the present fMRI study therefore was to directly investigate the neural correlates subserving the processing and integration of letters and speech sounds in early reading development. In contrast to previous developmental fMRI studies, we used a basic perceptual task in an attempt to study stimulus-induced rather than task-induced group differences in neural activity. The two reading groups in the present study were well-matched in age and educational standard, in order to control for maturational and experiential changes that otherwise might interfere with reading-related group effects. Importantly, the selected age range coincided with the earliest time in development at which reading disabilities are typically diagnosed and hence one of the earliest possible time-points to investigate the neurocognitive basis of letter-speech sound integration in dyslexia. An additional goal of the present study was to investigate whether and how potential letter-speech sound integration deficits in dyslexic children are related to the processing of those same stimuli presented in unisensory conditions. Given the proposed reciprocal connection between speech and print (Ziegler and Goswami, 2005), insights into the neurofunctional mechanisms supporting the visual, auditory and audiovisual aspects of letter-speech sound processing seem critical for understanding the emergence of reading difficulties in dyslexia. Finally, we examined whether the neural processing of letters, speech sounds and their combination correlated with behavioral performance on reading and reading-related tasks to further probe the hypothesis that a neural deficit in letter-speech sound integration is indeed predictive of reading failure. 


\section{METHODS}

\section{PARTICIPANTS}

Eighteen children with a diagnosis of dyslexia (mean age: 9.39, standard deviation (sd): $0.43,1$ female) and 16 children without reading impairment (mean age: 9.43, sd: $0.44,4$ female) participated in the study. All subjects were healthy, righthanded, native-Dutch speakers with normal or corrected-to-normal vision and normal audition. Dyslexic subjects were recruited via the Dutch Regional Institute for Dyslexia (RID) and diagnosed using an extensive, standardized cognitivebehavioral test procedure. Non-impaired readers were recruited via local schools. The groups were matched for educational level, age, handedness and IQ (Wechsler Intelligence Scale estimated IQ within 1 SD of norm, $\geq 85$ ) and were tested on various measures for reading status using a standard test battery (Blomert and Vaessen, 2009). These include a computerized reading test consisting of three levels of difficulty (high-frequency, low-frequency and pseudowords), in which subjects are required to read as many words as possible within 1.5 minutes. It further included a phoneme-deletion task in order to assess subjects' phonological abilities, a decoding (i.e. spelling) task and a letter-to-sound matching task. Criteria for dyslexia were based on a discrepancy score between performance on the reading test and $I Q$, with children with a standard reading score within the lower $10^{\text {th }}$ percentile, but average IQ score were classified as dyslexic (see results section, table 1). Informed consent was obtained from children and parents, in accordance with the local ethical guidelines.

\section{STIMULI AND TASK DESIGN}

Stimuli were visual letters and auditory speech sounds corresponding to Dutch single letters (consonants: b, d, g, h, k, I, n, p, r, s, t, z; vowels: a, e, i, y, o, u) adapted from (van Atteveldt et al., 2004). Stimuli were presented using the software Presentation (Neurobehavioral Systems, Albany, US) in blocks corresponding to four experimental conditions: unisensory letters, unisensory speech sounds, multisensory congruent letter-speech sound pairs, multisensory incongruent letterspeech sound pairs. During multisensory stimulation, stimuli were presented simultaneously. The experiment included four experimental runs, eight blocks and nine fixation periods each. One block (20.8 s) consisted of four miniblocks (see 'image acquisition'). Each block contained 16 stimuli (4 per miniblock) and was repeated twice per run, resulting in 128 stimuli per condition. The order of blocks was pseudorandomized within runs and the order of runs was counterbalanced across subjects. Children were instructed to carefully listen to the speech sounds and/or view the letters. To ensure that children attended the stimuli, a line drawing ('nemo'-fish) a voice (saying 'nemo') or a combination of the two was presented 
(8/128 trials or every 45 seconds on average) pseudo-randomized. Children were instructed to detect the stimuli by pressing a button.

\section{IMAGE ACQUISITION}

All children were acquainted to the scanning environment and trained to hold still using a simulation scanner. In the actual fMRI session, blood-oxygen-leveldependent (BOLD) signals were measured using a 3 Tesla Siemens headscanner (Allegra; Erlangen, Germany). Functional MRI data were acquired using a T2*sensitive gradient echo planar imaging (EPI) sequence covering the whole-brain (24 slices, slice-thickness $4.5 \mathrm{~mm}, 3 \times 3$ in-plane resolution, repetition time $(T R)=5.2 \mathrm{~s}$, TRslice/Echo Time (TE) $=63 / 32 \mathrm{~ms}$, Field of View (FOV): $192 \mathrm{~mm}^{2}$, matrix size: $64 \times 64 \times 24)$. Volume acquisition time was $1.5 \mathrm{~s}$ followed by a silent delay of $3.7 \mathrm{~s}$ in which stimuli were presented, resulting in a total TR of $5.2 \mathrm{~s}$. The long inter-scan delay was used to minimize the effects of scanning noise on experimental activation (van Atteveldt et al., 2004). A high resolution T1-weighted anatomical image (voxel size: $1 \times 1 \times 1 \mathrm{~mm}^{3}$ ) was acquired for each subject using a three dimensional gradient echo sequence (ADNI-MPRAGE, 192 slices, $1 \mathrm{~mm}$ slice-thickness, TR $=2.25$ $\mathrm{s}, \mathrm{TE}=2.6 \mathrm{~ms}, \mathrm{FA}=9$ degrees, matrix size: $256 \times 256$ ) optimized for morphometric analyses of MRI data across platforms (Jack et al., 2008).

\section{FMRI DATA ANALYSIS AND STATISTICS}

Imaging data were analyzed using BrainVoyager QX (BrainInnovation, Maastricht, the Netherlands). Functional data were preprocessed to correct for slice scan time differences (using sinc interpolation), 3D motion artifacts (trilinear interpolation), linear drifts, and low-frequency non-linear drifts (high pass filter $\leq 3 \mathrm{cycles} /$ time course). No spatial or additional temporal smoothing was applied. Functional data were then coregistered with the anatomical volume and transferred into standard stereotaxic space using Talairach normalization (Talairach and Tournoux, 1988).

Statistical maps were generated by modeling the evoked hemodynamic response for all four conditions (letters, speech sounds, congruent letter-speech sound pairs, incongruent letter-speech sound pairs) as boxcars convolved with a two-gamma hemodynamic response function in the context of the General Linear Model (GLM). Population-level inferences concerning BOLD signal changes between the experimental conditions were based on a random effects model with predictors separated for each subject. Statistical comparisons between conditions were based on \%-normalized beta values.

The first GLM was a single-factor model including the four conditions as separate predictors (GLM1) used to determine brain regions involved in processing unisensory and multisensory processing across all subjects. Moreover, two separate GLM's were computed for fluent and dyslexic readers in order to evaluate the spatial pattern of activation in each reading group separately (GLM2 \& 3, Figure 1). 
The beta values from GLM1 served as input for the calculation of statistical comparisons based on reading ability. Brain regions sensitive to the interactions between reading status and letter-speech sound congruency were of particular interest as they reflect the differential processing of the learned letter-speech sound association between fluent and dyslexic readers. To test this hypothesis, a 2x2 factorial model including 'reading status' (fluent, dyslexic) and 'multisensory condition' (congruent, incongruent) was computed including the interactions between the two factors (GLM4). A corresponding analysis including the 'unisensory conditions' was used to compare dyslexic and fluent readers on the processing of letters and speech sounds in isolation (GLM 5). All between-group comparisons were restricted to voxels activated by either of the unisensory conditions in isolation (Visual > Baseline OR Auditory > Baseline) by application of a functional mask.

Two statistical criteria were used to identify an area as multisensory integration site: the congruency effect defined as the difference between congruent and incongruent letter-speech sound pairs (e.g., van Atteveldt et al., 2007) and the Multisensory Interaction Index (MSI), defined as the increase/decrease of activation in multisensory (MS) conditions relative to the largest unisensory (US) response in each subject $(\mathrm{MSI}=((\mathrm{MS}-\mathrm{US} \max ) / \mathrm{USmax}) * 100)$ (Meredith and Stein, 1986; Wallace et al., 1992).

Depending on the specificity of the contrast, multisubject statistical maps were corrected for multiple comparisons using the false-discovery rate (Genovese et al., 2002) or cluster-size thresholding (Forman et al., 1995; Goebel et al., 2006). For GLM4 (i.e., the interaction between 'multisensory condition' and 'reading status'), an initial voxel-level threshold was set to $p<.01(t=5.1)$ uncorrected resulting in a cluster level of $115 \mathrm{~mm}^{3}$ after 1000 iterations and a corresponding corrected falsepositive probability of $5 \%$ or less. For GLM5 (i.e., the direct comparisons between control and dyslexic readers on the auditory or visual condition), an initial voxellevel threshold was set to $\mathrm{p}<.01(\mathrm{t}=2.5)$ uncorrected, resulting in a minimum cluster of 168 voxels at a false-positive probability of $5 \%$ or less.

As a final step in the analysis, we calculated correlations between the fMRI response and behavioral performance measures on reading-related tasks. Two performance measures were selected for visualization: reading and speed on letter-speech sound matching. Both tasks reliably distinguished fluent from dyslexic readers (see table 1). Letter-speech sound matching was added to the reading measure because the stimuli corresponded closely to those presented during scanning. Furthermore, dyslexic readers were equally accurate on letter-speech sound matching but showed a reliable difference in processing speed. Based on the assumption that faster processing is one of the main indices for the automation of a cognitive process (Schneider and Chein, 2003), correlations of a reaction time measure with the fMRI response could provide evidence for differences in the automation of letter-speech sound integration between reading groups. Behavioral data used for correlation-analysis were corrected for outliers, defined as values deviating more than two standard deviations (SD) from the mean. Rejected data 
points were replaced by the closest maximum or minimum value. Unless otherwise indicated all correlation coefficients were calculated using linear correlation statistics (Pearson's R). In addition, we used stepwise multiple linear regressions in order to determine which of the $\mathrm{FMRI}$ group effects explains a significant portion of independent variance in reading performance. Independent variables in this analysis were \% signal change values related to a) the congruency effect in PT/HS and STS, b) the response to speech sounds in aSTG, PT/HS and STS, and c) the response to visual letters in FG. In order to assess co-linearity, the variance inflation factor (VIF) was computed for each variable. For all independent variables the VIF was between 1.05 and 2.29 and hence multi co-linearity was considered of no concern for the present model.

\section{BEHAVIORAL RESULTS}

\section{ATTENTION TASK}

Both reading groups performed at ceiling for the detection of visual, auditory and audiovisual attention control stimuli (Fluent: mean=97.91, SEM=1.55; Dyslexic: mean=99.08, SEM=0.55). In addition, non-impaired and dyslexic readers responded equally fast to attention stimuli (Fluent: mean $=575 \mathrm{~ms}, \mathrm{SEM}=16.1$; Dyslexic: mean=609 ms, SEM=14.0; $p=.10$ ), indicating that attention levels were comparable across reading groups.

\section{OFFLINE BEHAVIOR/DIAGNOSTIC TESTING}

As can be seen in table 1, the performance of dyslexic children on reading-related tasks outside the scanner was poor compared to their fluently-reading peers. Specifically, dyslexic readers showed impaired reading (within the lower $10^{\text {th }}$ percentile on a standardized test of word reading) and poor performance on subtests involving phonological awareness and phonological decoding (Table 1). 


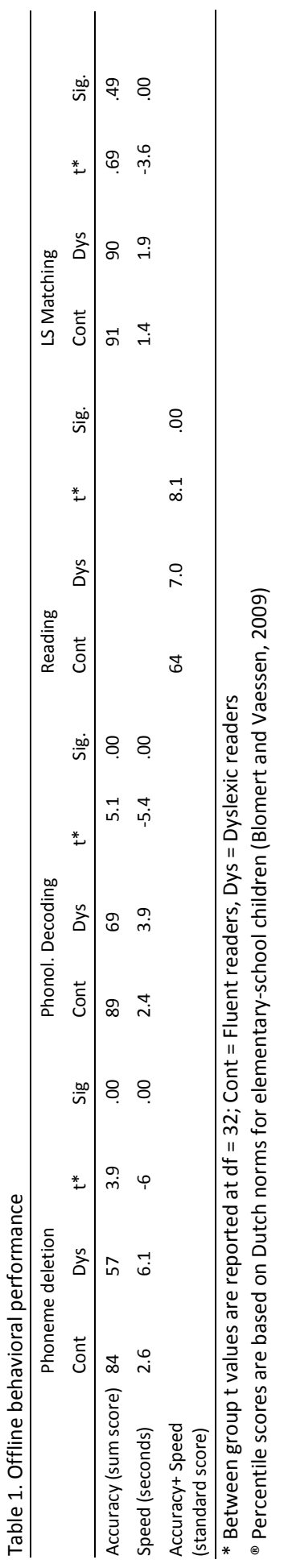




\section{IMAGING RESULTS}

\section{UNISENSORY ACTIVATIONS}

In order to assess the basic networks involved in the processing of speech sounds and letters presented in isolation, we compared visual and auditory conditions against baseline in each reading group. Figure 1 provides an overview of the spatial cortical network involved in viewing letters (red) and listening to speech sounds (yellow) for fluently-reading and dyslexic children. Orange brain regions represent convergence zones where activity for visual and auditory stimuli overlapped.

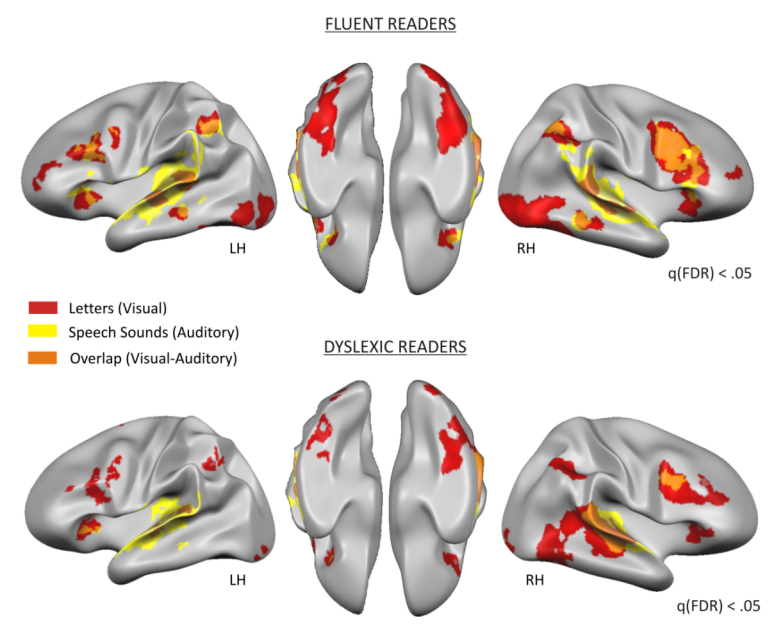

Figure 1. Spatial cortical networks involved in processing letters (red), speech sounds (yellow), or both unisensory conditions (orange) in fluent (upper panel) and dyslexic readers (lower panel).

Between-groups statistical comparisons revealed two brain regions that were differentially activated for processing unisensory stimuli in fluent compared to dyslexic readers (Figure 2). Dyslexic children showed weaker activity for processing speech sounds in the anterior superior temporal gyrus (aSTG) (Figure 2a) and for processing letters in an area around the fusiform gyrus (FG) bilaterally (see table 2; Figure 2b). Interestingly, dyslexics also displayed less activity for speech sounds in this same FG (visual letter) area. No other area of activation survived the correction for cluster size. 

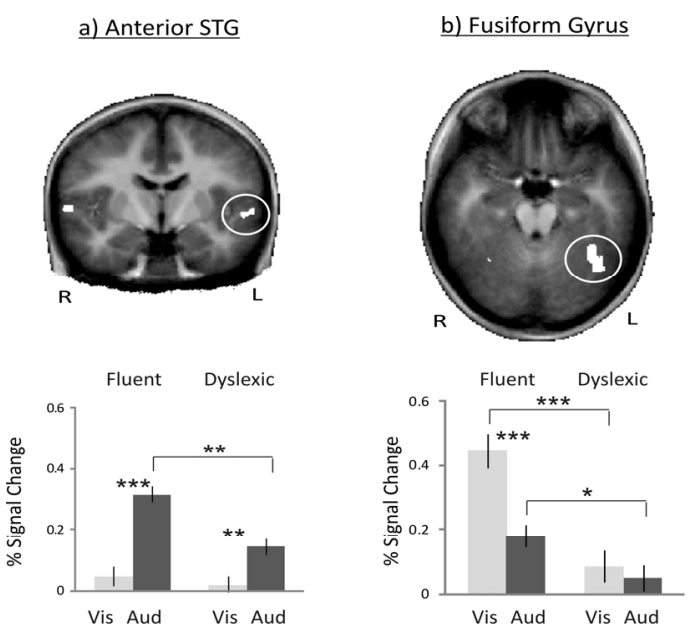

Figure 2. Mean percent signal change and standard error of mean (SEM) are shown for the unisensory group effects in the left anterior superior temporal gyrus (A), and the left fusiform gyrus (B) projected on the average anatomy $(\mathrm{N}=34)$.

\section{MULTISENSORY ACTIVATIONS}

The further goal of the analysis was to identify areas for the integration of letters and speech sounds in dyslexic vs. fluent readers by computing the interaction between 'reading status' and 'multisensory condition' (congruent vs. incongruent letter-speech sound pairs). The results revealed a significant interaction in the dorsal part of the left superior temporal gyrus (Planum Temporale, PT) in close proximity to primary auditory cortex (Heschl Sulcus, HS) (Table 2). An investigation of the time courses in PT/HS revealed the presence of a strong effect of multisensory congruency in fluent readers (Figure 3 , top right). This effect was absent in the dyslexic group.

Next we assessed how the unisensory conditions contributed to the observed between-group congruency difference. Figure 3a (bar graphs) shows the average fMRI signal change in the PT/HS interaction cluster for the multisensory and unisensory conditions in both reading groups. The PT/HS exhibits a clear auditoryspecific activation profile in both reading groups. However, the absolute strength of the auditory response was reduced in dyslexia. In the presence of such a unisensory group effect, it is suitable to normalize the multisensory response to account for differences in unisensory processing for each individual when determining letter-speech sound integration. This can be achieved by using the Multisensory Interaction Index (MSI = ((MS-USmax)/USmax)*100), where positive values reflect response enhancement whereas negative MSI values reflect response suppression (Meredith and Stein, 1986; Wallace et al., 1992). This analysis revealed that dyslexic readers failed to suppress activity to speech sounds in PT/HS when these are presented in combination with incongruent letters (Figure 4). In contrast, fluent 
readers clearly show this suppression effect. None of the reading groups exhibited an enhancement of the response to congruent multisensory stimuli compared to the maximal response evoked by the unisensory conditions.

In addition to PT/HS, a bilateral cluster in superior temporal sulcus (STS) showed a significant group-by-congruency interaction (Table 2). Both activation-clusters were located within the middle portion of STS (Figure 3b). Comparable to PT/HS, fluent readers activated STS more for the presentation of congruent as opposed to incongruent letter-speech sound pairs, while dyslexic readers showed no congruency effect. In relation to the auditory response, dyslexic readers did not show any significant modulation in the multisensory conditions based on letter-speech sound congruency. In line with this observation, dyslexic readers failed to show a multisensory suppression effect in STS for incongruent letter-speech sound pairs (Figure 4). No main effect of reading ability was found for the processing of multisensory letter-speech sound pairs. Table 2 provides a summary of all group effects.

Table 2. ROI details and statistics per analysis

\begin{tabular}{llrrrrrrl}
\hline \multicolumn{1}{c}{ Brain area Hemisphere } & \multicolumn{3}{c}{ Talairach Coordinates } & Voxels & \multicolumn{2}{c}{ Effect size } & \multicolumn{2}{l}{ Statistical test } \\
& & $\mathrm{X}$ & $\mathrm{Y}$ & $\mathrm{Z}$ & \multicolumn{5}{c}{ F, t-value } & $\mathrm{p}$-value \\
\hline PT/HS & Left & -42 & -28 & 13 & 117 & 16.70 & .00 & Interaction Group*Congruency \\
STS & Left & -56 & -33 & 4 & 171 & 7.73 & .01 & \\
STS & Right & 58 & -33 & 3 & 225 & 8.34 & .01 & \\
aSTG & Left & -51 & -8 & 1 & 171 & 2.89 & .01 & Control AUDITORY - Dyslexic AUDITORY \\
aSTG & Right & 57 & -8 & 7 & 169 & 2.80 & .01 & \\
FG & Left & -36 & -51 & -17 & 315 & 3.83 & .00 & Control VISUAL - Dyslexic VISUAL \\
FG & Right & 36 & -55 & -11 & 268 & 4.51 & .00 & \\
\hline
\end{tabular}

* Average $t$ value and $p$ value across all voxels in a ROI

** Statistical tests used for ROI selection (corrected for cluster size at alpha $=5 \%$ ) 

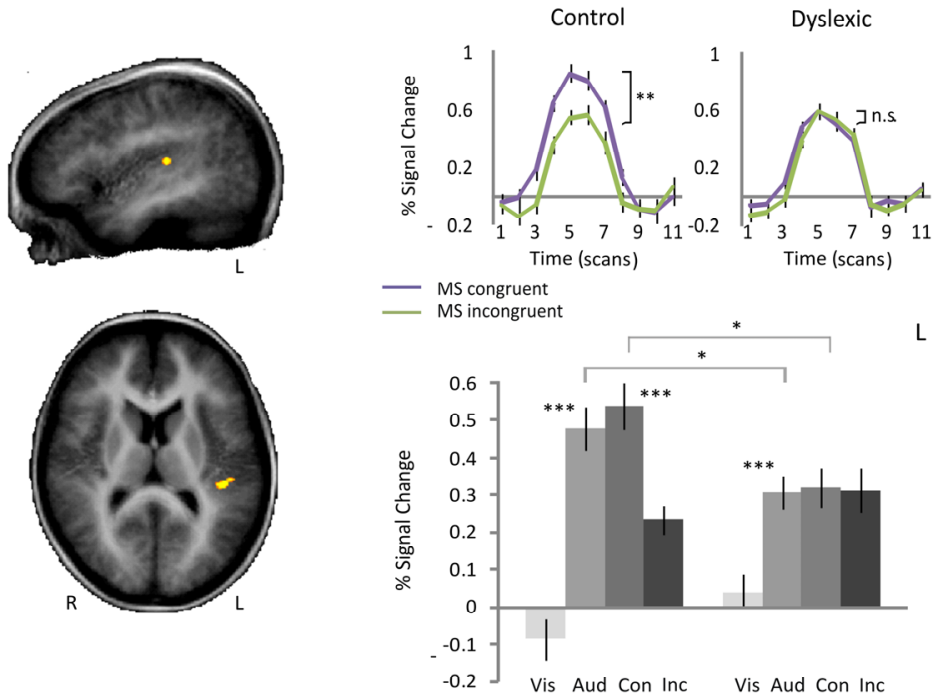

\section{Superior Temporal Sulcus}
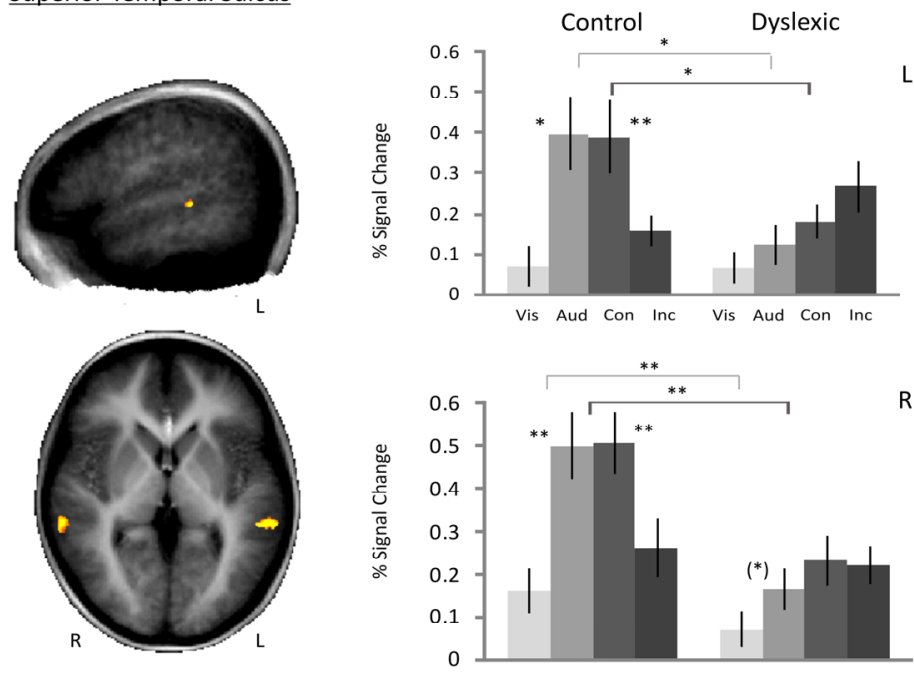

Figure 3. Interaction effect between 'reading group' and 'multisensory condition' in the Planum Temporale/Heschl Sulcus of the left hemisphere (A) projected on the average anatomy. The right side of the figure depicts the percent signal change and corresponding SEM as a function of time for fluent and dyslexic readers in MS congruent (purple line) and MS incongruent (green line) conditions. Bar graphs illustrate the percent signal change and SEM for the multisensory and unisensory conditions in fluent (left) and dyslexic readers (right) (based on mean \% signal change per subject).

The interaction site in the left and right superior temporal sulci is shown in (B). Bar graphs illustrate the mean percent signal change in each condition for fluent (left) and dyslexic readers (right) corresponding to the left (top) and right hemispheres (bottom). 


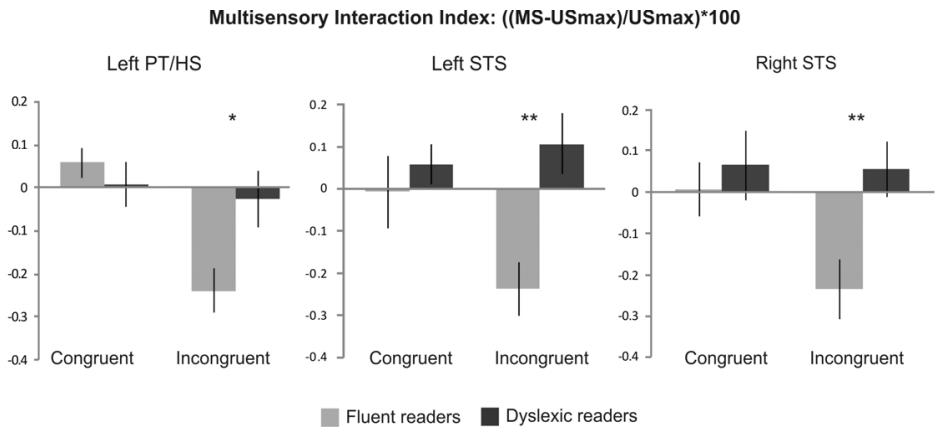

Figure 4. Mean BOLD responses and SEM for both reading groups indicate a strongly reduced suppression in the dyslexics for incongruent letter-speech sound pairs relative to the maximal unisensory response (USmax) in all brain areas identified in the interaction analysis (Left PT/HS, Left and Right STS).

\section{CORRELATION BETWEEN UNISENSORY AND MULTISENSORY CORTICAL RESPONSES}

Unisensory responses to speech sounds correlated positively with the congruency in PT/HS (R PARTIAL $=.67, p=.000)$ and the left STS (R PARTIAL $=.41, p=.019$ ) even after the factor reading ability was partialled out. Moreover, the visual response in left and right FG correlated with congruency effects in PT/HS (left FG: R = $.38, p=.025$; right $F G: R=.37, p=.030$ ), with fluent readers showing stronger responses to letters and a stronger effect of letter-speech sound congruency. This effect was non-significant when the factor reading group was partialled out, indicating that it was dependent upon reading ability.

\section{CORRELATIONS WITH PERFORMANCE}

We calculated full and partial correlations (controlling for factor group) between the $\mathrm{fMRI}$ response in left PT/HS, STS, aSTG and FG with reading-related measures (Figure 5). Two performance measures were selected for visualization: reading and speed on letter-speech sound matching (see methods). Figure 5 illustrates that the fMRI congruency response in PT/HS and STS correlated positively with accuracy on the reading task and negatively with reaction times on letter-speech sound matching (Figure 5a, b). Moreover, the auditory response in aSTG correlated with reading accuracy and the speed of letter-speech sound matching (Figure $5 \mathrm{c}$ ) as did the visual response in FG (Figure $5 \mathrm{~d}$ ). All reported correlations turned out to be nonsignificant when the factor reading group was controlled using partial correlations, suggesting that the brain-behavior correlations were driven by group differences in reading ability.

We used stepwise and hierarchical multiple linear regression to further evaluate whether the neural responses that correlated strongest with reading performance were the same as the ones explaining independent variance in reading. This analysis included the \%-signal change values of all relevant brain areas as predictor 
variables (Congruency effect: PT/HS, STS; Auditory response: PT/HS, STS, aSTG; Visual response: $\mathrm{FG}$ ). In the absence of high co-linearity (see methods), the congruency effect in PT/HS as well as the visual response in FG were found to both cause significant reductions in error variance on the reading task (PT/HS: $R=.51$, $R$ square change $=.27, \mathrm{~F}=12.04 ; \mathrm{p}=.002 ; \mathrm{FG}: \mathrm{R}=.52$, Combined model: $\mathrm{R}=.62$; $\mathrm{R}$ square change $=.11, \mathrm{~F}=5.73 ; \mathrm{p}=.023)$. Together, these two neural effects explained almost $40 \%$ of the variance in reading performance $(R$ square $=.39$ ). Auditory effects explained no additional variance that was not already explained by the effect of congruency in PT/HS and STS, while the congruency effect explained an additional $20 \%$ of the variance above and beyond the auditory response to speech. The visual response to letters in FG explained about $11 \%$ additional variance beyond the variance already explained through the auditory response and the congruency effect in PT/HS. 
Reading Task

A) Left Planum Temporale/Heschl Sulcus

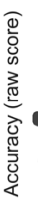

$-0.5$ tMRI Congruency Etfect (\% Signal Change)

B) Left Superior Temporal Sulcus

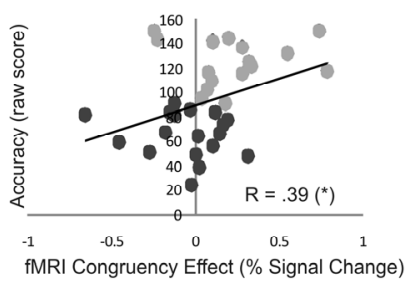

C) Left Anterior Superior Temporal Gyrus
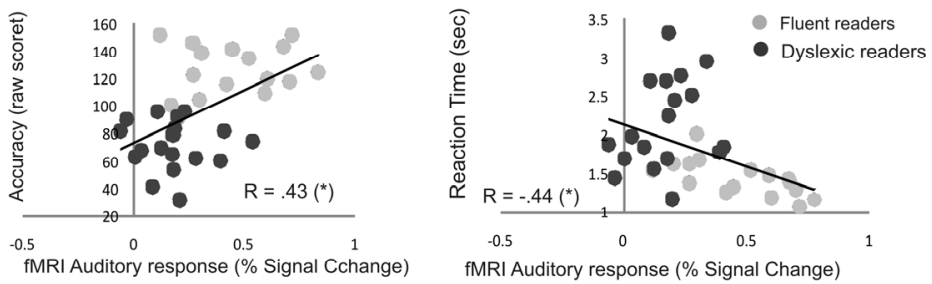

D) Left FusIform Gyrus

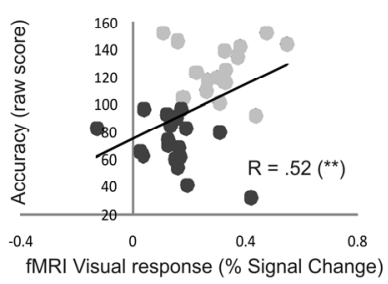

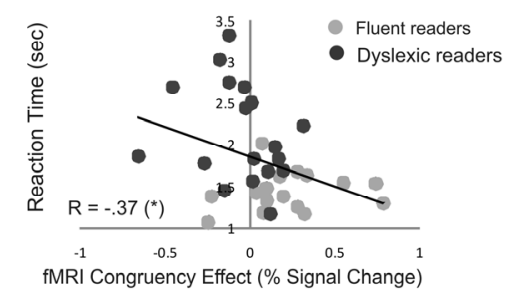

Letter-Sound Matching Task

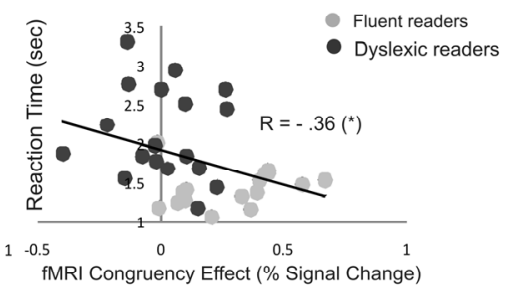

Figure 5. Correlations between reading accuracy as well as speed of letter-speech sound matching and the neural response to congruent vs. incongruent letter-speech sound pairs in PT/HS (fMRI congruency effect) (A), the fMRI congruency effect for incongruent letter-speech sound pairs in STS (B), the auditory response in the aSTG (C) and the visual response in FG (D). 


\section{DISCUSSION}

The main goal of the present study was to investigate whether dyslexic children differ from fluent readers in the processing of letters, speech sounds and their combinations. In multisensory conditions, we found weaker effects of congruency in PT/HS and STS of dyslexic children, indicating less successful integration of letters and speech sounds. This effect was accompanied by weaker activation in response to unisensory speech sounds in dyslexic readers in PT/HS, STS, aSTG and weaker activation to unisensory visual letters in FG. The congruency effect in PT/HS and the visual response to letters were, moreover, found to both explain a significant and (largely) independent part of the individual variance in reading performance. Finally, we examined the relation between unisensory and multisensory group effects. We found that the response to speech sounds in PT/HS and STS as well as the visual response in FG correlated with the strength of the congruency effect in PT/HS. Moreover, to investigate how uni- and multisensory effects are related, we calculated the multisensory interaction index (MSI). The data revealed that fluent readers strongly suppress activity to multisensory incongruent letter-speech sound stimuli, while dyslexic readers show no such suppression effect.

\section{MULTISENSORY PROCESSING OF LETTERS AND SPEECH SOUNDS}

The present neuroimaging study revealed that dyslexic children differ from fluently-reading children in the neural integration of basic letter-speech sound pairs. Fluently-reading children activate PT/HS and STS more strongly for the processing of congruent compared to incongruent letter-speech sound pairs. In contrast, dyslexic children exhibit little or no modulation of cortical responses to speech sounds in auditory cortex (PT/HS) and superior temporal cortex (STS) as a function of audiovisual congruency. As indicated by their adequate accuracy on matching letters and speech sounds on offline behavioral tasks, this deficit could not be explained by dyslexic readers' insufficient knowledge about letter-speech sound correspondences. As the congruency between letters and speech sounds cannot be established unless auditory and visual inputs have been successfully integrated (Van Atteveldt et al., 2007), reduced congruency effects in dyslexic children are likely to indicate less successful letter-speech sound integration. This finding extends earlier neuroimaging investigations in adult dyslexic readers by showing that a deficit in letter-speech sound integration is an emergent property of learning to read and not the result of a lifetime of reading difficulties (Blau et al., 2009). Moreover, it suggests that the ability to efficiently integrate letters with speech sounds in PT/HS and STS might be one of the direct neurofunctional correlates of reading failure.

Our finding that PT/HS and STS were involved in the integration of letters and speech sounds is in line with previous results in healthy adults (van Atteveldt et al., 2004; van Atteveldt et al., 2007). STS is a well-known heteromodal structure that 
receives input from multiple senses via cortical and subcortical connections (Beauchamp et al., 2004; Macaluso et al., 2004). PT/HS activation, by comparison, has been generally associated with the processing of speech and complex sounds (Binder et al., 1996; Seifritz et al., 2002). In addition, activation in PT has also been related to integration of spoken and written language (Nakada et al., 2001; van Atteveldt et al., 2004), and the learning of new audiovisual associations (Hasegawa et al., 2004).

The present correlation between congruency effects in PT/HS and left STS and performance on reading-related tasks point to auditory and superior temporal brain structures as potential neuroanatomical correlates linking letter-sound integration and reading skill. More concretely, weaker congruency effects in PT/HS as well as STS of dyslexic children were associated with lower reading scores, while stronger congruency effects in fluent readers were associated with higher reading scores. These correlations turned out to be non-significant when the factor reading group was partialled out indicating that they indeed reflect an effect of reading ability. In addition, neural integration responses in PT/HS and STS correlated with the speed of performance on letter-speech sound matching, in the absence of accuracy differences for judging the congruency between letters and sounds. Given that the speed of processing is one of the major indices of the automation of a cognitive process (Schneider and Chein, 2003), this finding indicates that a neural deficit in letter-speech sound integration reflects an inability to quickly retrieve or apply knowledge about letter-speech sound associations during reading. This is supported by findings from behavioral (Blomert and Vaessen, 2009) and electrophysiological studies (Froyen et al., 2009) suggesting a dissociation between accuracy and speed in the learning of letter-speech sound associations during development. Although unstable letter-speech sound associations have been suggested as a potential key factor in dyslexia (Ehri, 2005; Share, 1995), this hypothesis has remained largely untested. The present study provides first time empirical support for PT/HS and STS as neuroanatomical correlates for a failure to adequately automate letter-speech sound processing skills in dyslexic children.

\section{UNISENSORY PROCESSING AND EFFECTS OF READING ABILITY}

Next to investigating the multisensory integration of letters and speech sounds, the present study examined whether dyslexic children differ from fluent readers in the processing of unisensory letters and speech sounds. Overall, our results demonstrate that both dyslexic and fluently-reading children activated a spatially similar network of brain regions for processing letters and speech sounds, in good agreement with previous findings (Blau et al., 2009; van Atteveldt et al., 2004). Nevertheless, group differences for processing unisensory stimuli between dyslexic and fluent readers were observed, localized to PT/HS and STS (interaction sites) and two additional processing regions in the anterior part of superior temporal gyrus (aSTG) and the fusiform gyrus (FG). The finding that dyslexic subjects underactivate superior tempo- 
ral brain regions when processing speech sounds is in line with previous pediatric neuroimaging studies that implicated perisylvian cortex including the left superior temporal gyrus (Temple et al., 2001), middle temporal gyrus (Cao et al., 2006; Hoeft et al., 2007), and angular gyrus/supramarginal gyri (Shaywitz et al., 2002) using more complex phonological tasks. In addition, the neural responses to visual letters in the fusiform gyrus were less pronounced in the dyslexic group. The location of the FG activation was in close proximity to areas previously implicated for the processing of letters or words (Cohen and Dehaene, 2004; Cohen et al., 2002; Flowers et al., 2004; McCandliss et al., 2003). In line with the present results, electrophysiological recordings in dyslexic adults and children have shown that responses for letter-strings in occipito-temporal cortex were reduced in dyslexic readers (Helenius et al., 1999; Maurer et al., 2007). It is interesting to note that in the present data-set, the relatively weak response to speech sounds in the fusiform cortex was also weaker in dyslexic subjects than in fluent readers, potentially indicating weaker crossmodal modulations across auditory and visual sensory cortices.

\section{RELATION BETWEEN UNISENSORY AND MULTISENSORY EFFECTS}

Lastly, we examined whether and how unisensory and multisensory neural deficits in dyslexic readers are related in order to better understand their interactive contribution to reading skill. The present data revealed that the congruency effect in PT/HS and STS is positively correlated with the neural response to speech sounds, indicating the dependency between phonological processing of speech and letter-speech sound integration in beginning readers. Moreover, we found significant correlations between the unisensory response to visual letters in FG and the congruency effect in PT/HS that as a function of reading ability, suggesting a further association between visual responses to print and letter-speech sound integration. Together, the correlation of the visual response and the auditory response with letter-speech sound integration in PT/HS make a case for the existence of an interactive cortical network involved in linking orthographic and phonological representations of print in early reading development. Therefore, we think that the present findings may be best accounted for by reading models that emphasize the reciprocal nature between reading and phonological development. Strong supporting evidence for the relevance of visual and audiovisual neural responses for reading was gathered using multiple linear regressions. Together, the visual FG response to letters and the congruency effect in PT/HS explained almost $40 \%$ of the variance in individual reading performance. The phonological response to speech sounds in PT/HS in contrast was also relevant, but did not explain more variance in reading than the congruency effect alone or in combination with the visual response. In other words, the influence of the auditory response to speech sounds on reading performance was mediated through its relation to visual letters. While these linear regression results should be treated with caution because of the small sample size of neuroimaging studies, they certainly 
indicate a dominant role for letter-speech sound integration and visual processing in early reading performance.

As a last step in the analysis, we assessed the nature of the relations between unisensory and multisensory responses by use of the multisensory interaction index (see methods). We found strong suppression effects in PT/HS and STS to incongruent letter-speech sound pairs in fluent readers, which were absent in dyslexic readers (for similar effects in adults see Blau et al., 2009). Such effects most likely reflect the downregulation of neural activity to speech sounds in the presence of incongruent orthographic inputs. This suppression finding may reflect a neural mechanism to prevent the learning of irrelevant multisensory associations in typical readers. Analogously, the absence of such an effect in dyslexia may represent an insufficient filtering of irrelevant letter-speech sound associations during reading acquisition. None of the reading groups showed a multisensory enhancement effect for congruent letter-speech sound pairs as previously reported for adult experienced readers (van Atteveldt et al., 2004). This could be related to the fairly strong unisensory responses to auditory stimulation, by which the likelihood of observing multisensory enhancement effects is reduced (Standford et al., 2005).

\section{CONCLUSION}

In sum, the present data provide first evidence for a neural deficit in the integration of letters and speech sounds in dyslexic children localized to auditory cortex and the superior temporal sulcus. These neurofunctional effects closely resemble those seen in adult dyslexia. This suggests that letter-speech sound integration is an emergent property of learning to read that develops inadequately in dyslexic readers, presumably as a result of a deviant interactive specialization of neural systems for processing auditory and visual linguistic inputs.

Acknowledgements: This research was supported by the European Union, Sixth Framework Program (NeuroDys) awarded to L.B. and R.G. 


\section{REFERENCES}

Adams MJ. Beginning to Read: Thinking and Leaning about Print. Cambridge: MIT Press, 1994.

Beauchamp MS, Argall BD, Bodurka J, Duyn JH, Martin A. Unraveling multisensory integration: patchy organization within human STS multisensory cortex. Nat Neurosci 2004; 7: 1190-2.

Binder JR, Frost JA, Hammeke TA, Rao SM, Cox RW. Function of the left planum temporale in auditory and linguistic processing. Brain 1996; 119: 1239-47.

Blau V, van Atteveldt N, Ekkebus M, Goebel R, Blomert L. Reduced neural integration of letters and speech sounds links phonological and reading deficits in adult dyslexia. Curr Biol 2009; 19: $503-8$.

Blomert L. Dyslexie in Nederland. Amsterdam: Uitgeverij Nieuwezijds, 2005.

Blomert L, Vaessen A. 3DM Differential Diagnostics for Dyslexia: Cognitive Analysis of Reading and Spelling. Amsterdam: Boom Test Publishers, 2009.

Bradley L, Bryant PE. Categorizing sounds and learning to read - a causal connection. Nature 1983; 301: 419-21.

Cao F, Bitan T, Chou TL, Burman DD, Booth JR. Deficient orthographic and phonological representations in children with dyslexia revealed by brain activation patterns. J Child Psychol Psychiatry 2006; 47: 1041-50.

Cohen L, Dehaene S. Specialization within the ventral stream: the case for the visual word form area. Neuroimage 2004; 22: 466-76.

Cohen L, Lehericy S, Chochon F, Lemer C, Rivaud S, Dehaene S. Language-specific tuning of visual cortex? Functional properties of the Visual Word Form Area. Brain 2002; 125: 1054-69.

Ehri LC. Development of sight word reading: phases and findings. In: Snowling MJ and Hulme C, editors. The science of reading: a handbook. Oxford: Blackwell Publishing, 2005: 135-45.

Esser G, Schmidt MH, Woerner W. Epidemiology and course of psychiatric disorders in school-age children--results of a longitudinal study. J Child Psychol Psychiatry 1990; 31: 243-63.

Flowers DL, Jones K, Noble K, VanMeter J, Zeffiro TA, Wood FB, et al. Attention to single letters activates left extrastriate cortex. Neuroimage 2004; 21: 829-39.

Forman SD, Cohen JD, Fitzgerald M, Eddy WF, Mintun MA, Noll DC. Improved assessment of significant activation in functional magnetic resonance imaging ( $\mathrm{FMRI}$ ): use of a cluster-size threshold. Magn Reson Med 1995; 33: 636-47.

Froyen DJ, Bonte ML, van Atteveldt N, Blomert L. The long road to automation: neurocognitive development of letter-speech sound processing. J Cogn Neurosci 2009; 21: 567-80.

Genovese CR, Lazar NA, Nichols T. Thresholding of statistical maps in functional neuroimaging using the false discovery rate. Neuroimage 2002; 15: 870-8.

Goebel R, Esposito F, Formisano E. Analysis of functional image analysis contest (FIAC) data with Brainvoyager QX: From single-subject to cortically aligned group general linear model analysis and self-organizing group independent component analysis. Hum Brain Mapp 2006; 27: 392-401.

Hasegawa T, Matsuki K, Ueno T, Maeda Y, Matsue Y, Konishi Y, et al. Learned audio-visual multisensory associations in observed piano playing activate the left planum temporale. An fMRI study. Brain Res Cogn Brain Res 2004; 20: 510-8.

Helenius P, Tarkiainen A, Cornelissen P, Hansen PC, Salmelin R. Dissociation of normal feature analysis and deficient processing of letter-strings in dyslexic adults. Cereb Cortex 1999; 9: 476-83.

Hoeft F, Meyler A, Hernandez A, Juel C, Taylor-Hill H, Martindale JL, et al. Functional and morphometric brain dissociation between dyslexia and reading ability. Proc Natl Acad Sci U S A 2007; 104: 4234-9. 
Jack CR, Jr., Bernstein MA, Fox NC, Thompson P, Alexander G, Harvey D, et al. The Alzheimer's Disease Neuroimaging Initiative (ADNI): MRI methods. J Magn Reson Imaging 2008; 27: 68591.

Livingstone MS, Rosen GD, Drislane FW, Galaburda AM. Physiological and anatomical evidence for a magnocellular defect in developmental dyslexia. Proc Natl Acad Sci U S A 1991; 88: 7943-7.

Lyon GR, Shaywitz SE, Shaywitz BA. A definition of Dyslexia. Annals of Dyslexia 2003; 53: 1-14.

Macaluso E, George N, Dolan R, Spence C, Driver J. Spatial and temporal factors during processing of audiovisual speech: a PET study. Neuroimage 2004; 21 : 725-32.

Maisog JM, Einbinder ER, Flowers DL, Turkeltaub PE, Eden GF. A meta-analysis of functional neuroimaging studies of dyslexia. Ann N Y Acad Sci 2008; 1145: 237-59.

Maurer U, Brem S, Bucher K, Kranz F, Benz R, Steinhausen HC, et al. Impaired tuning of a fast occipito-temporal response for print in dyslexic children learning to read. Brain 2007; 130 : 3200-10.

McCandliss BD, Cohen L, Dehaene S. The visual word form area: expertise for reading in the fusiform gyrus. Trends Cogn Sci 2003; 7: 293-299.

Meredith MA, Stein BE. Visual, auditory, and somatosensory convergence on cells in superior colliculus results in multisensory integration. J Neurophysiol 1986; 56: 640-62.

Nakada T, Fujii Y, Yoneoka Y, Kwee IL. Planum temporale: where spoken and written language meet. Eur Neurol 2001; 46: 121-5.

Nicolson RI, Fawcett AJ, Dean P. Developmental dyslexia: the cerebellar deficit hypothesis. Trends Neurosci 2001; 24: 508-11.

Perfetti CA, Beck I, Bell L, Hughes C. Phonemic knowldege and learning to read are reciprocal: A longitudinal study of first grade children. Merill-Palmer Quarterly 1987; 33: 283-319.

Raij T, Uutela K, Hari R. Audiovisual integration of letters in the human brain. Neuron 2000; 28 : 617-25.

Ramus F. Developmental dyslexia: specific phonological deficit or general sensorimotor dysfunction? Curr Opin Neurobiol 2003; 13: 212-8.

Schneider W, Chein JM. Controlled and automatic processing: Behavior, theory and biological mechanisms. Cogntive Science 2003; 27: 525-559.

Seifritz E, Neuhoff JG, Bilecen D, Scheffler K, Mustovic H, Schachinger H, et al. Neural processing of auditory looming in the human brain. Curr Biol 2002; 12: 2147-51.

Share DL. Phonological recoding and self-teaching: Sine qua non of reading acquisition. Cognition 1995; 55: 151.

Shaywitz BA, Shaywitz SE, Pugh KR, Mencl WE, Fulbright RK, Skudlarski P, et al. Disruption of posterior brain systems for reading in children with developmental dyslexia. Biol Psychiatry 2002; 52: 101-10.

Shaywitz SE, Shaywitz BA. Dyslexia (specific reading disability). Biol Psychiatry 2005; 57: 1301-9.

Shaywitz SE, Shaywitz BA, Fletcher JM, Escobar MD. Prevalence of reading disability in boys and girls. Results of the Connecticut Longitudinal Study. Jama 1990; 264: 998-1002.

Snowling MJ. From language to reading and dyslexia. Dyslexia 2001; 7: 37-46.

Stanford TR, Quessy S, Stein BE. Evaluating the operations underlying multisensory integration in the cat superior colliculus. J Neurosci 2005; 25: 6499-508.

Stein J, Walsh V. To see but not to read; the magnocellular theory of dyslexia. Trends Neurosci 1997; 20: 147-52.

Talairach J, Tournoux P. Co-Planar Stereotactic Atlas of the Human Brain. Stuttgart: Thieme, 1988.

Tallal P, Miller S, Fitch RH. Neurobiological basis of speech: a case for the preeminence of temporal processing. Ann N Y Acad Sci 1993; 682: 27-47.

Temple E, Poldrack RA, Salidis J, Deutsch GK, Tallal P, Merzenich MM, et al. Disrupted neural responses to phonological and orthographic processing in dyslexic children: an fMRI study. Neuroreport 2001; 12: 299-307. 
Torgesen JK, Wagner RK, Rashotte CA. Longitudinal studies of phonological processing and reading. J Learn Disabil 1994; 27: 276-86.

van Atteveldt N, Formisano E, Goebel R, Blomert L. Integration of letters and speech sounds in the human brain. Neuron 2004; 43: 271-82.

van Atteveldt NM, Formisano E, Blomert L, Goebel R. The effect of temporal asynchrony on the multisensory integration of letters and speech sounds. Cereb Cortex 2007; 17: 962-74.

Vellutino FR, Fletcher JM, Snowling MJ, Scanlon DM. Specific reading disability (dyslexia): what have we learned in the past four decades? J Child Psychol Psychiatry 2004; 45: 2-40.

Wagner $\mathrm{R}$, Torgesen $\mathrm{J}$. The nature of phonological processes and its causal role in the acquisition of reading skills. Psychological Bulletin 1987; 101: 192-212.

Wallace MT, Meredith MA, Stein BE. Integration of multiple sensory modalities in cat cortex. Exp Brain Res 1992; 91: 484-8.

Ziegler JC, Goswami U. Reading acquisition, developmental dyslexia, and skilled reading across languages: a psycholinguistic grain size theory. Psychol Bull 2005; 131: 3-29. 

DEVIANT LETTER-SPEECH SOUND INTEGRATION IN DYSLEXIA: DEFICIT OR DEVELOPMENTAL DELAY?

Based on:

Blau, V., Goebel, R., Blomert, L. Deviant letter-speech sound integration in dyslexia: deficit or developmental delay? In preparation. 


\begin{abstract}
Developmental dyslexia has been associated with a core deficit in phonological processing, which is assumed to lead to reading problems through a deficit in learning or automating letter-speech sound associations. Using neuroimaging, we previously reported that dyslexic readers underactivate auditory/auditory association and superior temporal cortex for the integration of letters and speech sounds (congruency effect), as well as the processing of speech sounds in isolation (auditory effect). A critical question that emerged from these findings was whether reduced activation for unisensory and/or multisensory stimuli represents a developmental deficit or simply a delay on the developmental path towards fluent reading. To address this question, the present study compared a group of dyslexic adult readers to a group of fluent readers, who were either matched in chronological age or current reading level to the dyslexic group. The data reveal that both the auditory and the congruency effect are present in auditory/auditory association cortex when dyslexic readers are compared to a control group matched for chronological age, and hence replicates our previous results. The novel finding was that the reduced effect of congruency in auditory association cortex in dyslexia disappeared when the dyslexic group was compared to a younger control group matched for current reading level, indicating that reduced letter-speech sound integration relates to current reading ability rather than being a developmental deficit in dyslexia. Contrary to this, the reduced activation to speech sounds in the same brain regions was found in the chronological-age comparison and the reading-level comparison, indicating that the reduced activation for speech sound in dyslexic readers represents a dyslexia-specific deviance that cannot simply be accounted for by current reading ability. In conclusion, the present data suggest a dissociation between neural processes related to letter-speech sound integration related to current reading ability in dyslexia, and those related to unisensory processing of speech, which are more 'causally' related to dyslexia.
\end{abstract}




\section{INTRODUCTION}

Developmental dyslexia is a specific reading disorder characterized by slow and effortful reading and spelling in individuals who would be expected to have attained higher reading levels based on their age, motivation or other cognitive abilities (Lyon et al., 2003). Developmental dyslexia (hereafter referred to as "dyslexia") affects at least $4 \%$ of the population (Blomert, 2005; Esser et al., 1990; Shaywitz et al., 1990) and is best conceptualized as reflecting the lower tail of the normal distribution of reading ability (Shaywitz et al., 1992; Siegel, 2006). Advances in understanding the origin of dyslexia support an explanation in terms of a phonological processing deficit (Pennington et al., 1990; Ramus, 2003; Vellutino, 1978; Wagner and Torgesen, 1987), characterized by difficulties in perceiving, recognizing or manipulating the sounds structure of language (Vellutino et al., 2004). The understanding that spoken words are made up of constituent speech sounds is critical for the ability to associate those sounds with alphabetic letters (Bradley and Bryant, 1983; Byrne et al., 1996; Ehri, 2005), and hence crucial for becoming a fluent reader (Ehri, 2005). At the same time, awareness for individual speech sounds is likely to develop in response to reading instruction, because of the influence of letter-knowledge on phonological representations of speech (Ehri, 1992; Ehri and Wilce, 1980; Morais et al., 1987; Perfetti et al., 1987). Thus, the relation between phonological awareness for speech sounds and reading acquisition is likely to be reciprocal. This reciprocity predicts a central and mediating role for letter-speech sound associations in reading and reading failure.

Using functional Magnetic Resonance Imaging (fMRI), we recently investigated the neural correlates of letter- speech sound integration in dyslexic readers. Our results show that adult dyslexics underactivate an anterior region of auditory association cortex (anterior Superior Temporal Gyrus, STG, bordering Heschl Sulcus/Gyrus, $\mathrm{HS} / \mathrm{HG}$ ) for the integration of letter-speech sound stimuli as well as the processing of speech sounds in isolation (Blau et al., 2009). When we extended this study to dyslexic children, we again found reduced cortical activity related to the integration of letters and speech sounds in more posterior parts of the auditory/auditory association cortex (Planum Temporale, PT/HS) and superior temporal sulcus (STS) (Blau et al., in revision). Moreover, dyslexic children showed weaker neural activation related to the processing of unisensory speech sounds in PT/HS, STS and the anterior STG. Previous neuroimaging studies in dyslexic readers using readingrelated phonological tasks have also reported underactivation in dyslexia, predominantly localized to left hemisphere temporoparietal cortex (Brunswick et al., 1999; Horwitz et al., 1998; Paulesu et al., 2001; Rumsey, 1992; Temple, 2002).

Reduced neural activations for reading-related tasks in dyslexia are commonly thought to reflect a fundamental weakness in the reading process related to dyslexia and not just current reading ability (e.g. see Temple et al., 2002). However, most neuroimaging studies, including our own (Blau et al., 2009, in revision), compare dyslexic readers to a group of control subjects matched in chronological 
age, who are superior in their reading performance. As a result, reduced neural activation can either be the result of a fundamental weakness in the reading process or can be the consequence of the low reading skills in the dyslexic group (i.e. reduced experience with written language) (Backman et al., 1984; Bradley and Bryant, 1978).

In an attempt to overcome these difficulties, 'reading-level designs' have been employed, in which the dyslexic group is compared to a control group equated for current reading ability by being younger in chronological age (Backman et al., 1984). If no performance difference is found between the dyslexic group and the younger control group, then it is argued that dyslexic readers do not perform qualitatively different from fluent readers, but are instead simply delayed in their acquisition of reading skills. Similarly, if dyslexic subjects still differ from control readers in task-performance, although the control group has the same reading level, then it is assumed that dyslexic readers are qualitatively different from younger control readers. Behavioral research has used this design to indicate that impaired phonological processing in dyslexia reflects a processing deficit rather than a developmental delay (Bogliotti et al., 2008; Bradley and Bryant, 1978; Snowling et al., 2000; Sprenger-Charolles et al., 2000). Moreover, two recent neuroimaging studies have employed reading-level designs to study neural activation differences using phonological tasks. Hoeft and colleagues (Hoeft et al., 2006; Hoeft et al., 2007) compared a dyslexic group to a chronological-age control group as well as a younger control group equated for reading level on a rhyme judgment task. They showed that dyslexic children exhibit reduced activation in temporoparietal brain regions compared to both control groups, indicating that reduced activation in dyslexic children for processing phonological inputs is related to dyslexia itself rather than current reading level. Similarly, Schulz and colleagues (2009) recently provided evidence for the involvement of inferior parietal cortex in dyslexia-specific word processing during sentence reading.

The purpose of the present study was to utilize the logic of 'reading-level' designs to further explore the nature of our previously reported finding that dyslexic adult readers underactivate auditory/auditory association cortex for the integration of letters and speech sounds as well as for the processing of speech sounds in isolation. Given that the relation between awareness for speech sounds and reading acquisition is likely to be reciprocal (Ehri and Wilce, 1980; Perfetti et al., 1987), reduced neural responses related to letter-speech sound integration could reflect a dyslexia-specific deficit or a developmental delay that is accounted for by reading level per se. Similarly, reduced activation for speech sounds could be driven by reading ability or be a basic deficit in dyslexia. Differentiating between those neural processes in dyslexia that are 'symptomatic' to reading failure versus those that are more 'causal' could be important for a conclusive understanding of reading disorders.

In order to investigate these questions, we matched a subgroup of dyslexic adults scanned in the context of a previous larger fMRI study (Blau et al., 2009) to a 
younger group of control children, who were also part of a larger data-set (Blau et al., in revision) (Reading Level or RL comparison). At the same time, we selected a group of control adult subjects to serve as a second control group matched for chronological-age to the dyslexic group (Chronological Age or CA comparison). We hypothesize that if reduced activation for the integration of letters and speech sounds and/or unisensory speech sounds in dyslexic adults reflects a deficit related to dyslexia itself, then dyslexic readers should underactivate auditory/auditory association cortex for the chronological and the reading-level comparisons. However, if reduced letter-speech sound integration and/or unisensory processing of speech sounds relates to current reading ability rather than being a specific deficit in dyslexia, then reduced activation for the integration of letters and speech sounds should only be found for the chronological-age comparison but not for the readinglevel comparison.

\section{METHODS}

\section{PARTICIPANTS}

We selected the data of eight adult subjects and eight children without reading impairment, as well as eight adults with developmental dyslexia from previously acquired larger fMRI data-sets (see Blau et al., 2009; Blau et al., in revision). All subjects were screened for neurological and psychiatric disorders, were nativeDutch speakers, and had normal or corrected-to-normal vision as well as normal audition. Informed consent was obtained for all participants, which was voluntary and in accordance with the Maastricht University, Faculty of Psychology and Neuroscience, ethical guidelines.

\section{BEHAVIORAL EVALUATION}

Subjects were tested on reading and reading-related skills using a standard test battery (Blomert and Vaessen, 2009). Reading ability was measured using two comparable standardized tests of word and pseudoword reading, one computerized test (reading test of the 'differential diagnostic test battery for dyslexia' Maastricht; Blomert and Vaessen, 2009) and one paper-based test consisting of two parts ('one-minute reading test', Brus and Voeten, 1973; 'pseudoword reading test', Van den Bos et al., 1999). Both tests require subjects to read as many words as possible within a given amount of time. Criteria for dyslexia were met if subjects fell within the lower $10^{\text {th }} \%$ (c-score $\leq 2$ ) on the standardized test of reading (Table 1). Subjects were classified as controls if their reading score fell within or above $20^{\text {th }} \%$ of the average norm ( $c$-score $\geq 5$ ). Non-verbal intelligence was equated across subject groups as measured by the block-design and similarity subtests of the Wechsler Intelligence Scale for Children and Adults (WISC-R and Projectgroup, 1986). 


\section{SUBJECT GROUPS}

The present study consisted of three subject groups: one dyslexic group and two control groups. The dyslexic group (DYS-group) consisted of eight adults (1 female) who were classified as dyslexic using the abovementioned criteria. We then selected an additional, random sample of eight control subjects (2 female) that were matched to the DYS-group on chronological age (CA-group). In order to control for potential bias in the selection procedure we repeated this procedure to create a second CA group, so it could be verified that our statistical comparisons would not be influenced by the way control subjects has been selected. Importantly, we then also selected a second control group consisting of eight children (2 female) who were substantially younger than the DYS-group, but who had similar raw reading scores, i.e. who were matched for current reading level to the DYS group (RLgroup). Mean values of the critical variables and corresponding group contrasts ( $t-$ tests) are listed in Table 1.

Table 1. Group description

\begin{tabular}{|c|c|c|c|c|c|}
\hline & Adult Dyslexic (DYS) & Adult Control (CA) & Child Control (RL) & DYS vs. CA & DYS vs. RL \\
\hline $\mathrm{Nbr}$ of subjects & 8 & 8 & 8 & & \\
\hline Age (years, SEM) & $26.0,(1.3)$ & $23.25,(1.4)$ & $9.36,(.1)$ & .11 & $.00 * *$ \\
\hline Handedness & Right & Right & Right & & \\
\hline $\begin{array}{l}\text { Estimated } \\
\text { non-verbal IQ }\end{array}$ & $10.6,(.4)$ & $11.1,(1.2)$ & $10.9,(.7)$ & .34 & .33 \\
\hline \multicolumn{6}{|l|}{$\begin{array}{l}\text { Reading } \\
\text { (words/minute) }\end{array}$} \\
\hline $\begin{array}{l}\text {-raw score } \\
\text { (mean, SEM) }\end{array}$ & $108.6,(9.5)$ & $182.8,(8.6)$ & $110.8,(4.9)$ & $.00 * * *$ & .35 \\
\hline $\begin{array}{l}\text {-c-score } \\
\text { (mean, SEM) }\end{array}$ & $2.0,(.4)$ & $7.0,(.5)$ & $5.5,(.4)$ & $.00 * * *$ & $.00 * *$ \\
\hline
\end{tabular}

\section{STIMULI AND TASK DESIGN}

Stimuli were visual letters and auditory speech sounds corresponding to Dutch single letters (consonants: b, d, g, h, k, l, n, p, r, s, t, z; vowels: a, e, i, y, o, u) adapted from (van Atteveldt et al., 2004). Stimuli were presented using the software package Presentation (Neurobehavioral Systems, Albany, US) in blocks corresponding to four experimental conditions: unisensory letters, unisensory speech sounds, multisensory congruent letter-speech sound pairs, multisensory incongruent letter-speech sound pairs. During multisensory stimulation, stimuli were presented simultaneously. The experiment included four experimental runs, including eight experimental blocks and nine fixation periods each. One experimental block (20.8 s) was composed of four miniblocks (5.2 s). As the design had to be adapted for children to be performed in the scanner, stimulus presentations were slightly different between the adult and child studies. In the adult study each miniblock contained 5 stimuli ( 40 per condition and block), while in the child study each block 
contained only 4 stimuli (32 per condition and block). This adaptation was necessary in order to increase the inter-stimulus-interval to allow slower stimulus presentations in children. A pilot study in adult subjects using both set of parameters (from the child and adult study) confirmed that despite these adaptations the results obtained were highly comparable. All subjects were instructed to carefully listen to the speech sounds and/or view the letters. Children were additionally instructed to perform an attention-control task by pressing a button every time a line drawing ('nemo'-fish), a voice (saying 'nemo') or a combination of the two was presented ( $8 / 128$ trials or every 45 seconds on average). The order of blocks was pseudorandomized within runs and the order of runs was counterbalanced across subjects.

\section{IMAGE ACQUISITION, DATA ANALYSIS AND STATISTICS}

Blood-oxygen-level-dependent (BOLD) signals were measured using a 3 Tesla Siemens headscanner (Allegra; Erlangen, Germany). Functional MRI data were acquired using a $\mathrm{T} 2{ }^{*}$-sensitive gradient echo planar imaging (EPI) sequence covering the whole-brain (24 slices, slice-thickness $4.5 \mathrm{~mm}, 3 \times 3$ in-plane resolution, repetition time $(T R)=5.2 \mathrm{~s}$, TRslice/Echo Time $(T E)=63 / 32 \mathrm{~ms}$, Field of View (FOV): $192 \mathrm{~mm}^{2}$, matrix size: $\left.64 \times 64 \times 24\right)$. Volume acquisition time was $1.5 \mathrm{~s}$ followed by a silent delay of $3.7 \mathrm{~s}$ in which stimuli were presented, resulting in a TR of $5.2 \mathrm{~s}$. The long inter-scan delay was used to minimize the effects of scanning noise on experimental activation (Jancke et al., 2002). A high resolution T1-weighted anatomical image (voxel size: $1 \times 1 \times 1 \mathrm{~mm}^{3}$ ) was acquired for each subject using a three dimensional gradient echo sequence (ADNI-MPRAGE (child study) and MPRAGE (adult study), 192 slices, $1 \mathrm{~mm}$ slice-thickness, $1 \times 1$ in-plane resolution, TR $=2.25 \mathrm{~s}$, $\mathrm{TE}=2.6 \mathrm{~ms}, \mathrm{FA}=9$ degrees, matrix size: $256 \times 256$ ).

Imaging data were analyzed using BrainVoyager QX (BrainInnovation, Maastricht, the Netherlands; Goebel et al., 2006). Functional data were preprocessed to correct for slice scan time differences (using sinc interpolation), 3D motion artifacts (trilinear interpolation), linear drifts, and low-frequency non-linear drifts (high pass filter $\leq 3$ cycles/time course). No spatial or additional temporal smoothing was applied. Functional data were then coregistered with the anatomical volume and transferred into standard stereotaxic space using Talairach normalization (Talairach and Tournoux, 1988).

Statistical maps were generated by modeling the evoked hemodynamic response for all four conditions (letters, speech sounds, congruent letter-speech sound pairs, incongruent letter-speech sound pairs) as boxcars convolved with a two-gamma hemodynamic response function in the context of the General Linear Model (GLM). Population-level inferences concerning BOLD signal changes between the experimental conditions were based on a random effects model with predictors separated for each subject. Statistical comparisons between conditions were based on z-normalized beta values. In order to improve statistical power for making direct 
group comparisons, we used a Region of Interest (ROI)-based analysis approach, in which we focused on the auditory/auditory association cortex in the left hemisphere previously implicated in letter-speech sound integration in dyslexia (Blau et al., 2009, in revision). We selected cortical sites based on two functional contrasts across all subjects independent of group assignment (dyslexic or control): a) the contrast of speech sounds versus baseline, and b) the contrast congruent letterspeech sound pairs versus incongruent letter-speech sound pairs. These two contrasts were chosen because they allow us to investigate group differences between dyslexic and control readers with respect to the congruency effect and the auditory effect in a symmetric way, and hence allow controlling for potential biases in ROI selection. Previous knowledge about the location of congruency and auditory effects in fluent readers (van Atteveldt et al., 2004; van Atteveldt et al., 2007) as well as anatomical knowledge (Durvernoy, 1999) further helped to validate the ROI locations.

Using the functional contrast of speech sound versus baseline, we defined the following three ROI's (hereafter called 'auditory ROI's'): posterior auditory/auditory association cortex including the Planum Temporale (PT) (posterior auditory ROI), the area around Heschl sulcus (HS) extending into Heschl's gyrus (HG) (middle auditory ROI), and anterior auditory/auditory association cortex on superior temporal gyrus just anterior to HG (anterior auditory ROI). The z-normalized beta values were extracted in each of the ROI's for each subject and condition. We then performed two univariate analyses of variance (ANOVAs), with dependent variables being either the congruency effect or the auditory effect. The independent variables were reading group (adult control, adult dyslexic, child control), and auditory ROI-position (anterior, middle, posterior).

Using the functional contrast of congruent versus incongruent letter-speech sound pairs we additionally defined an ROI covering the left Heschl Gyrus extending medially into the transverse sulcus (hereafter called 'congruency ROI'). The congruency ROI partly overlapped with the middle auditory ROI as defined in the previous analysis but was shifted slightly more medially (white circle in Figure 1). The znormalized beta values were extracted for each ROI, subject and condition. Independent t-tests were used to compare reading groups (adult control vs. adult dyslexic, child control vs. adult dyslexic) on the strength of the observed auditory and congruency effect.

\section{RESULTS}

\section{AUDITORY ROI'S:}

Congruency effect: the data revealed a main effect of group (adult control, adult dyslexic, child control) on the neural response to congruent versus incongruent letter-speech sound pairs (congruency effect) (Table 2). This effect was independent of the ROI position within auditory/auditory association cortex (anterior, 
middle, posterior) as indicated by the absence of a significant interaction between group and position. Moreover, no main effect of ROI-position was found. We therefore considered it safe to collapse the data across all three auditory cortical sites before proceeding to a more detailed investigation of the main effect of reading group. Post-hoc pairwise comparisons revealed that the influence of reading group on the effect of congruency in auditory/auditory association cortex was characterized by a significant reduction of the congruency effect in the DYSgroup compared to CA control group (chronological-age comparison). Importantly, no difference in the congruency effect between dyslexic and control readers was found once the DYS-group was compared to the RL control group (reading-level comparison) (Figure 1). In order to ensure that the chronological-age comparison was not the result of the way control adult readers were selected from the larger sample of control subjects, we repeated the analysis using a second, randomlydrawn group of control adult readers. Again, we found a significant reduction of the congruency effect in dyslexic adults in the chronological-age comparison (mean difference $=.32, \mathrm{t}=4.12, \mathrm{p}<.001$ )

Auditory effect: a second univariate ANOVA was performed to investigate the influence of reading groups and ROI-position on the auditory response to speech sounds versus baseline in the auditory/auditory association cortex. Analogously to what we found in the previous analysis, we found a main effect of group on the neural response to unisensory presented speech sounds (auditory effect) (Table 2). This effect was independent of the ROI position within auditory/auditory association cortex (no group-by-position interaction), and no main effect of ROI-position was found. Post-hoc pairwise comparisons on the mean auditory response per reading group revealed a significant reduction of the auditory effect in dyslexic adults compared to the CA-group (Table 2). This comparison remained statistically significant when another sample of CA subjects was used as control group (mean difference $=.30, t=4.45, p<.000$ ). However, unlike what we found for the congruency effect the reduced auditory effect in dyslexic adults was preserved even when dyslexic readers were compared to the RL-group (Figure1).

\section{CONGRUENCY ROI:}

The main finding of the auditory ROI analysis was that the reduced activation for speech sounds in the auditory cortical ROI's of dyslexic readers was not eliminated in the reading-age comparison, unlike the effect of congruency. Since we based the selection of the relevant ROI's on the functional contrast between unisensory speech sounds versus baseline, one might argue that the present preservation of the auditory effect could partly be a consequence of the way the ROI's had been selected (although this selection was unbiased in the sense that it included all subjects, while the ROI analysis focused on between-group differences of reading status). In order to examine this possibility, we repeated the CA comparison and the RL comparisons for the congruency and the auditory effects using the congru- 
ency ROI. We first compared dyslexic readers to the CA control group on their congruency and auditory responses. As expected, this analysis revealed a significant reduction of activity in the DYS-group for the congruency effect and the auditory effect (Table 2). We then compared the DYS-group to the RL control group. In line with our previous findings, the data show that the reduction in the neural effects of congruency is eliminated by the reading-age comparison, while the reduction in the auditory response to speech sounds is preserved (Table 2).
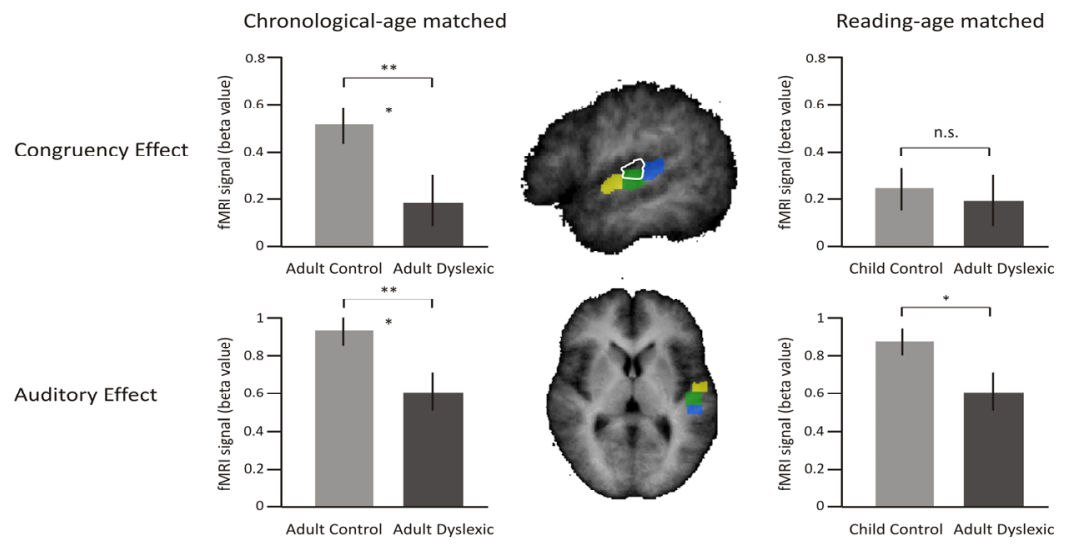

Figure 1. The averaged $\mathrm{fMRI}$ signal (z-normalized beta values) and corresponding standard error of the mean (SEM) across three regions of interest in the left auditory/auditory association cortex (yellow=anterior STG/HG, green=HG/HS, blue=PT/HS) for the chronological-age comparison (left) and the reading-age comparison (right). The upper half of the figure depicts the fMRI response for the congruency effect (congruent versus incongruent letter-speech sound pairs), while the lower half of the figure depicts the $\mathrm{fMRI}$ response to unisensory auditory stimulation (speech sound versus baseline). While the chronological-age comparison revealed significantly reduced effects of congruency and reduced effects of processing unisensory speech sounds in dyslexic readers, only the reduced effect of unisensory processing of speech sounds remained when dyslexic and control readers were equated for reading-age. 


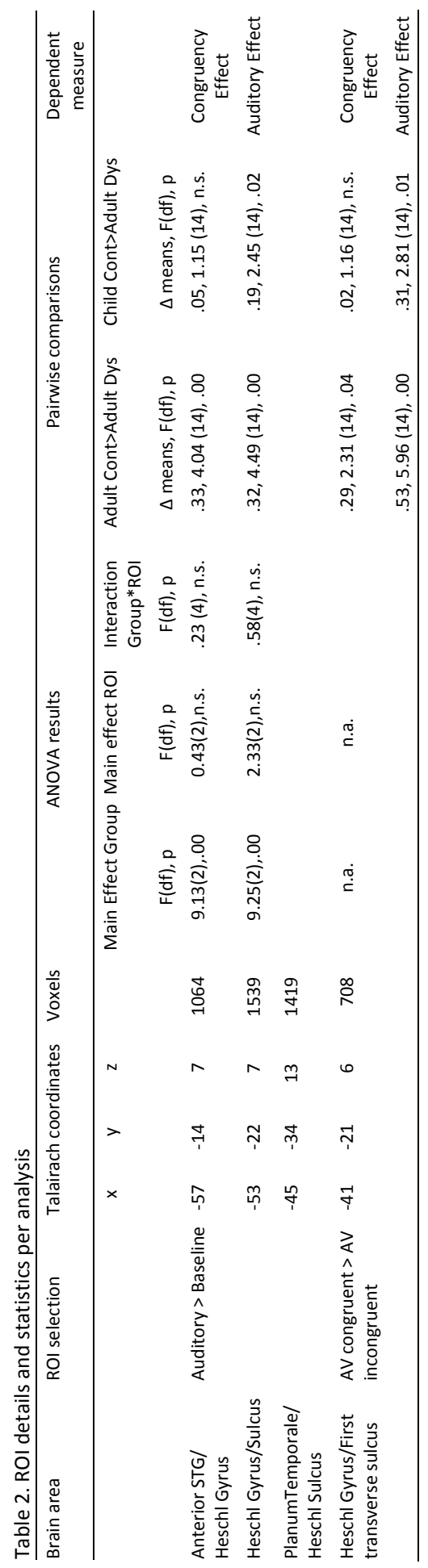




\section{DISCUSSION}

The aim of the present investigation was to further explore the nature of our previously observed findings that dyslexic readers underactivate auditory/auditory association cortex for the integration of letter-speech sound pairs and the processing of unisensory presented speech sounds (Blau et al., 2009; in revision). Focusing on activation differences in the auditory/auditory association cortex, we compared a group of dyslexic adult readers to a group of fluent readers, who were either matched in chronological age or reading level to the dyslexic group. The data reveal that both the auditory and the congruency effect are present when dyslexic readers are compared to a control group matched for chronological age, and hence replicates what we reported previously using larger fMRI data-sets (Blau et al., 2009, in revision). The novel finding was that the reduced effect of congruency in auditory/auditory association cortex in dyslexia disappeared when the dyslexic group was compared to a younger control group matched for current reading level, indicating that reduced letter-speech sound integration relates to current reading ability rather than being a fundamental weakness in dyslexia. Contrary to this, the reduced activation to speech sounds in the same brain regions was found to persist independent of whether dyslexic readers were compared to chronological-age matched or reading-level controls. This indicates that the reduced activation for speech sound in dyslexic readers represents a dyslexia-specific deviance that cannot simply be accounted for by current reading ability.

Reading research often assumes that a phonological processing impairment in dyslexia leads to reading failure through a deficit in processing letter-speech sound associations (Vellutino et al., 2004). In two previous neuroimaging studies we provided empirical evidence for this claim by showing that dyslexic readers underactivate the auditory/auditory association and superior temporal cortex for letterspeech sound integration and the unisensory processing of speech sounds (Blau et al., in revision; Blau et al., 2009). Because reading ultimately starts with the processing of visual information about print while reading difficulties in dyslexia have mainly been related to a phonological processing deficit, we interpreted the finding of reduced letter-speech sound integration in dyslexia as reflecting a proximate cause for reading failure (e.g. Blau et al., in revision). The present finding that the congruency difference in auditory/auditory association cortex is eliminated in the reading-level comparison supports this conclusion, because it suggests the existence of a strong dependency between letter-speech sound integration and current reading ability. Even in typically developing readers, improvements in reading skills have been associated with changes in brain activation in the absence of any reading deficit (Schlaggar et al., 2002; Turkeltaub et al., 2003). Hence, the present nullfinding in the reading-level comparison with respect to the congruency effect may be interpreted to reflect a substantial developmental delay that is not qualitatively different from the typical developmental trajectory in reading (Backman et al., 1984). 
A reduction in the auditory responses to speech sounds in dyslexic readers was found in the chronological-age and the reading-level comparisons, indicating that reduced phonological processing of speech sounds in dyslexia represents a deficit rather than a developmental delay. Previous neuroimaging work using more complex phonological tasks has also shown that underactivation in temporoparietal brain regions of dyslexic subjects persist in reading-level comparisons (Hoeft et al., 2006, 2007). In addition, several behavioral investigations have previously used reading-level designs to provide evidence for a phonological deficit in dyslexia (Bogliotti et al., 2008; Bowey et al., 1992; Bradley and Bryant, 1978; SprengerCharolles et al., 2000) in line with the commonly accepted 'phonological-deficit' hypothesis (Pennington et al., 1990; Ramus, 2003; Vellutino, 1978; Wagner and Torgesen, 1987). The fact that we observe dyslexia-specific activation differences in auditory/auditory association areas situated more anteriorly than previously reported effects in temporoparietal cortex could reflect the absence of lexicalsemantic retrieval when processing speech sounds in isolation compared to more complex stimuli, such as words (Binder et al., 2000).

It should be noted that previous investigations using reading-level designs have often compared older dyslexic children to younger dyslexic children (e.g. Hoeft et al., 2006, 2007; Schulz et al., 2009). The present data show that a reduced response to speech sounds in dyslexic readers holds even if adult dyslexic readers are compared to elementary-school children. This suggests that the origin of the deviant development of phonological representations for speech sounds in adult dyslexic readers is likely to originate early in reading development. In line with this, some studies have reported speech perception deficits in young children with a familial risk for dyslexia (Gerrits and de Bree, 2009) and even infants with at-risk status (Richardson et al., 2003). However, other studies failed to show significant differences in speech perception before the start of reading instruction (Boets et al., 2007; Boets et al., 2006), in line with the proposed fragile nature of speech perception problems in dyslexia (Blomert and Mitterer, 2004).

One question that arises from the fact that dyslexic adult readers were matched in their absolute reading score to a group of younger control children is how it is possible that they achieve this despite their reading impairment. In previous research, reading performance in dyslexic subjects has been linked to overactivation in regions such as the inferior frontal gyrus (e.g. Brunswick, 1999), presumably reflecting compensatory activity in dyslexia as a means of accounting for a lifetime of poor reading (e.g., through a greater reliance on articulatory processes). The use of a ROI-based analysis approach in the present study did not allow us to investigate this question. However, as adult dyslexic readers may represent a group of highly compensated readers, the question about potential compensatory mechanisms could be a relevant one. To nonetheless address this question, we performed an additional whole-brain analysis next to the main ROI-based analysis, in which we explored potential differences between the processing of congruent vs. incongruent letter-speech sound pairs. We found no brain areas that were activated more 
strongly in adult dyslexia as compared to the RL or CA control group. This absence of such neural overactivation in dyslexic readers may be explained through the simple nature of the present stimuli and task, as opposed to more complex phonological tasks employed previously (Brunswick et al., 1999; Horwitz et al., 1998; Paulesu et al., 2001; Rumsey, 1992; Temple, 2002). Another possible explanation is that compensatory neural processes in dyslexia may not necessarily be reduced to single hotspots of activation, but may as well be characterized by more subtle differences, such as synchronized sub-threshold activity in a network of brain regions, which may not be easily detectable using fMRI. Such an interpretation would be in line with a recent meta-analyses, in which overactivation in frontal brain regions was characterized as one of the most inconsistent finding in the current literature on reading disorders (Maisog et al., 2008).

While the reading-level comparison in the present study eliminated the multisensory congruency effect, the auditory effect remained significant despite the matching for reading ability between dyslexic and younger control readers. This result suggests that our previously observed effects of reduced activation for unisensory speech sounds and letter-speech sound integration in dyslexia, which always occurred together (Blau et al., 2009, in revision), can at least partly be dissociated. The letter-speech sound integration response seems to be more closely related to reading ability than the phonological response to speech sounds. In a broader context, the differential involvement of neural processes related to letter-speech sound integration versus speech-sound processing in dyslexia might best be explained when the connection between a phonological core deficit in dyslexia and the actual reading problem (i.e. the fast identification of visual words) is viewed as a continuous developmental path. On this path, the neural integration of letters and speech sounds may be situated proximate to the actual reading problem, while the neural processing of speech sounds might be more distant to reading but at the same time more 'causal' for explaining reading failure. A full understanding of the neurobiology of dyslexia is likely to require the integration of both, distal and proximate explanations for reading failure.

Acknowledgements: This research was partly supported by the European Union, Sixth Framework Program (NeuroDys) to L.B. and R.G., and the Dutch Health Care Insurance Board (CVZ 608/001/2005) to L.B. 


\section{REFERENCES}

Backman JE, Mamen M, Ferguson HB. Reading level design: conceptual and methodological issues in reading research. Psychol Bull 1984; 96: 560-8.

Binder JR, Frost JA, Hammeke TA, Bellgowan PS, Springer JA, Kaufman JN, et al. Human temporal lobe activation by speech and nonspeech sounds. Cereb Cortex 2000; 10: 512-28.

Blau V, Reithler J, Van Atteveldt N, Seitz J, Gerretsen P, Goebel R, et al. Deviant processing of letters and speech sounds as proximate cause of reading failure: an fMRI study of dyslexic children. in revision.

Blau V, van Atteveldt N, Ekkebus M, Goebel R, Blomert L. Reduced neural integration of letters and speech sounds links phonological and reading deficits in adult dyslexia. Curr Biol 2009; 19: 503-8.

Blomert L. Dyslexie in Nederland. Amsterdam: Uitgeverij Nieuwezijds, 2005.

Blomert L, Mitterer $\mathrm{H}$. The fragile nature of the speech-perception deficit in dyslexia: natural vs synthetic speech. Brain Lang 2004; 89: 21-6.

Blomert L, Vaessen A. 3DM Differential Diagnostics for Dyslexia: Cognitive Analysis of Reading and Spelling. Amsterdam: Boom Test Publishers, 2009.

Boets $B$, Ghesquiere $P$, van Wieringen $A$, Wouters J. Speech perception in preschoolers at family risk for dyslexia: relations with low-level auditory processing and phonological ability. Brain Lang 2007; 101: 19-30.

Boets B, Wouters J, van Wieringen A, Ghesquiere P. Auditory temporal information processing in preschool children at family risk for dyslexia: relations with phonological abilities and developing literacy skills. Brain Lang 2006; 97: 64-79.

Bogliotti C, Serniclaes W, Messaoud-Galusi S, Sprenger-Charolles L. Discrimination of speech sounds by children with dyslexia: comparisons with chronological age and reading level controls. J Exp Child Psychol 2008; 101: 137-55.

Bowey JA, Cain MT, Ryan SM. A reading-level design study of phonological skills underlying fourthgrade children's word reading difficulties. Child Dev 1992; 63: 999-1011.

Bradley L, Bryant PE. Difficulties in auditory organisation as a possible cause of reading backwardness. Nature 1978; 271: 746-7.

Bradley L, Bryant PE. Categorizing sounds and learning to read - a causal connection. Nature 1983; 301: 419-21.

Brunswick N, McCrory E, Price CJ, Frith CD, Frith U. Explicit and implicit processing of words and pseudowords by adult developmental dyslexics: A search for Wernicke's Wortschatz? Brain 1999; 122 ( Pt 10): 1901-17.

Brus BT, Voeten MJM. Een-minuuttest, vorm A en B. Nijmegen: Berkhout, 1973.

Byrne ME, Crowe TA, Hale ST, Meek EE, Epps D. Metalinguistic and pragmatic abilities of participants in adult literacy programs. J Commun Disord 1996; 29: 37-49.

Durvernoy HM. The Human Brain: Surface, Three-Dimensional Sectional Anatomy with MRI, and Blood Supply. Wien: Springer, 1999.

Ehri LC. Reconceptualizing the development of sight word reading and its relationship to recoding. In: Gough LE, Ehri LC and Treiman R, editors. Reading Acquisition. Hillsdale, NJ: Erlbaum Publishers, 1992.

Ehri LC. Development of sight word reading:phases and findings. In: Snowling MJ and Hulme C, editors. The science of reading: a handbook. Oxford: Blackwell Publishing, 2005: 135-45.

Ehri LC, Wilce LS. The influence of orthography on readers' conceptualization of phonemic structure of words. Applied Psycholinguistics 1980: 371-85.

Esser G, Schmidt MH, Woerner W. Epidemiology and course of psychiatric disorders in school-age children--results of a longitudinal study. J Child Psychol Psychiatry 1990; 31: 243-63. 
Gerrits E, de Bree E. Early language development of children at familial risk of dyslexia: speech perception and production. J Commun Disord 2009; 42: 180-94.

Goebel R, Esposito F, Formisano E. Analysis of functional image analysis contest (FIAC) data with Brainvoyager QX: From single-subject to cortically aligned group general linear model analysis and self-organizing group independent component analysis. Hum Brain Mapp 2006; 27 : 392-401.

Hoeft F, Hernandez A, McMillon G, Taylor-Hill H, Martindale JL, Meyler A, et al. Neural basis of dyslexia: a comparison between dyslexic and nondyslexic children equated for reading ability. J Neurosci 2006; 26: 10700-8.

Hoeft F, Meyler A, Hernandez A, Juel C, Taylor-Hill H, Martindale JL, et al. Functional and morphometric brain dissociation between dyslexia and reading ability. Proc Natl Acad Sci U S A 2007; 104: 4234-9.

Horwitz B, Rumsey JM, Donohue BC. Functional connectivity of the angular gyrus in normal reading and dyslexia. Proc Natl Acad Sci U S A 1998; 95: 8939-44.

Jancke L, Wustenberg T, Scheich $\mathrm{H}$, Heinze HJ. Phonetic perception and the temporal cortex. Neuroimage 2002; 15: 733-46.

Lyon GR, Shaywitz SE, Shaywitz BA. A definition of Dyslexia. Annals of Dyslexia 2003; 53: 1-14.

Morais J, Castro SL, Scliar-Cabral L, Kolinsky R, Content A. The effects of literacy on the recognition of dichotic words. Q J Exp Psychol A 1987; 39: 451-65.

Paulesu E, Demonet JF, Fazio F, McCrory E, Chanoine V, Brunswick N, et al. Dyslexia: cultural diversity and biological unity. Science 2001; 291: 2165-7.

Pennington BF, Van Orden GC, Smith SD, Green PA, Haith MM. Phonological processing skills and deficits in adult dyslexics. Child Dev 1990; 61: 1753-78.

Perfetti CA, Beck I, Bell L, Hughes C. Phonemic knowledge and learning to read are reciprocal: A longitudinal study of first grade children. Merill-Palmer Quarterly 1987; 33: 283-319.

Ramus F. Developmental dyslexia: specific phonological deficit or general sensorimotor dysfunction? Curr Opin Neurobiol 2003; 13: 212-8.

Rumsey JM. Failure to activate left temporoparietal cortex in dyslexia. Archives of Neurology 1992; 49: 527-534.

Schlaggar BL, Brown TT, Lugar HM, Visscher KM, Miezin FM, Petersen SE. Functional neuroanatomical differences between adults and school-age children in the processing of single words. Science 2002; 296: 1476-9.

Schulz E, Maurer U, van der Mark S, Bucher K, Brem S, Martin E, et al. Reading for meaning in dyslexic and young children: Distinct neural pathways but common endpoints. Neuropsychologia 2009.

Shaywitz SE, Escobar MD, Shaywitz BA, Fletcher JM, Makuch R. Evidence that dyslexia may represent the lower tail of a normal distribution of reading ability. N Engl J Med 1992; 326: 145-50.

Shaywitz SE, Shaywitz BA, Fletcher JM, Escobar MD. Prevalence of reading disability in boys and girls. Results of the Connecticut Longitudinal Study. Jama 1990; 264: 998-1002.

Siegel LS. Perspectives on dyslexia. Paediatr Child Health 2006; 11: 581-7.

Snowling M, Bishop DV, Stothard SE. Is preschool language impairment a risk factor for dyslexia in adolescence? J Child Psychol Psychiatry 2000; 41: 587-600.

Sprenger-Charolles L, Cole P, Lacert P, Serniclaes W. On subtypes of developmental dyslexia: evidence from processing time and accuracy scores. Can J Exp Psychol 2000; 54: 87-104.

Talairach J, Tournoux P. Co-Planar Stereotactic Atlas of the Human Brain. Stuttgart: Thieme, 1988.

Temple E. Brain mechanisms in normal and dyslexic readers. Curr Opin Neurobiol 2002; 12: 17883.

Turkeltaub PE, Gareau L, Flowers DL, Zeffiro TA, Eden GF. Development of neural mechanisms for reading. Nat Neurosci 2003; 6: 767-73. 
van Atteveldt N, Formisano E, Goebel R, Blomert L. Integration of letters and speech sounds in the human brain. Neuron 2004; 43: 271-82.

van Atteveldt NM, Formisano E, Blomert L, Goebel R. The effect of temporal asynchrony on the multisensory integration of letters and speech sounds. Cereb Cortex 2007; 17: 962-74.

Van den Bos KP, Lutje Spelberg HC, Scheepsma AJM, De Vries JR. De Klepel: pseudowoordentest. Lisse: Swets \& Zeitlinger, 1999.

Vellutino FR. Dyslexia: Theory and Research. Cambridge: MIT Press, 1978.

Vellutino FR, Fletcher JM, Snowling MJ, Scanlon DM. Specific reading disability (dyslexia): what have we learned in the past four decades? J Child Psychol Psychiatry 2004; 45: 2-40.

Wagner $\mathrm{R}$, Torgesen $\mathrm{J}$. The nature of phonological processes and its causal role in the acquisition of reading skills. Psychological Bulletin 1987; 101: 192-212.

WISC-R, Projectgroep. WISC-R: Nederlandstalige uitgave. Lisse: Swets \& Zeitlinger, 1986. 

Chapter 6

GENERAL DISCUSSION 
This thesis set out to examine the neural basis of reading skill and dyslexia through the lens of basic cognitive operations of integrating speech sound information with visual letter information. The following paragraphs will discuss the results and their implications across experimental studies, starting from the overall question what the study of letter-speech sound integration may contribute to our understanding about reading and reading disabilities.

\subsection{NEURAL SUBSTRATES FOR PROCESSING LETTERS AND SPEECH SOUNDS IN FLUENT AND DYSLEXIC READERS}

Learning and automating letter-speech sound associations has long been assigned a central role in learning to read (Ehri, 2005) and reading difficulties (Vellutino, 1978; Vellutino et al., 2004), but to date this assumption largely evaded empirical support. In experiments described in this thesis used fMRI to localize a network of brain regions that was differentially activated for the processing of letter-speech sound pairs in dyslexic adults and children when compared to their fluently-reading peers (chapter 3 through 5). While fluent readers activated a range of auditory and heteromodal brain regions, including PT/HS, STG as well as STS for the integration of letters and speech sounds, dyslexic readers showed reduced activation in several of these cortical structures. Figure 1 summarizes these main areas of differential neural activity between fluent and dyslexic readers in a schematic way. Adult dyslexic readers were found to underactivate a portion of the auditory cortex on the STG for the processing of congruent vs. incongruent letter-speech sound pairs when compared to fluently-reading adults (red circle in Figure 1; chapter 3).

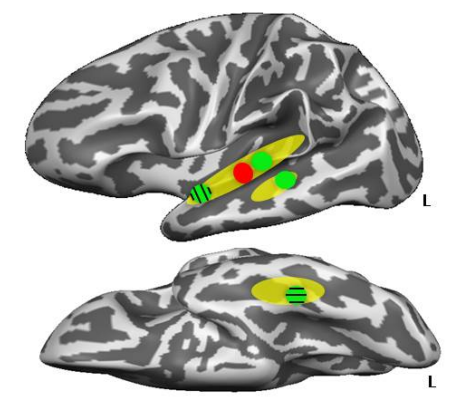

Figure 1. Schematic summary of brain structures for letter-speech sound integration in fluent readers (yellow), and areas showing deviant cortical responses in dyslexic adults (red) and children (green). Activations are illustrated on the unfolded cortical mesh of an individual anatomy (lateral view is = top, medial view $=$ bottom).

By comparison, dyslexic children were found to underactivate a region of auditory cortex slightly more posterior on PT/HS for the integration of letter-speech sound pairs, as well as heteromodal STS (green circles - no lines; chapter 4). Both of these structures were also implicated in literate adults for the integration of letters and 
speech sounds under implicit task conditions (chapter 2). In addition to reduced neural integration, both dyslexic adults and children showed reduced activation for the processing of unisensory presented speech sounds. In adults, this unisensory underactivation was localized to STG (red circle) while in children it was localized to PT/HS, STS (green circles - no lines) and a very anterior region on STG (green circle vertical lines). Dyslexic children, in addition, exhibited reduced activation for the processing of unisensory presented visual letters in FG (green circle - horizontal lines), a structure that was also activated in the literate adult brain for the processing of letters and their integration with speech sounds (chapter 2). Taken together, these results clearly support two main assumptions: first, letter-speech sound pairs are processed differently in the dyslexic brain, and secondly, dyslexic readers suffer from impaired phonological processing of speech inputs. The following paragraphs aim to interpret both findings in relation to reading and reading failure.

\subsubsection{ALTERED PROCESSING OF LETTER-SPEECH SOUND PAIRS IN DYSLEXIA}

For the interpretation of the first result (i.e., reduced letter-speech sound integration in the brain of dyslexic subjects), an important finding was that the fMRI integration response in dyslexia was directly associated with subjects' impaired performance on reading-related tasks (chapter 4 and 5). Weaker neural integration responses were associated with a fewer number of correctly read words on a standardized test of reading (chapter 4). Furthermore, the effect of congruency in PT/HS explained $27 \%$ of the individual variance in reading performance in chapter 4 , a finding that was further corroborated by the data from chapter 5 . Here, it was found that the reduction in letter-speech sound integration in auditory and auditory/auditory association cortex of dyslexic adult readers disappeared when dyslexic readers were matched to a younger group of control readers with the same absolute reading score. The elimination of the congruency difference in auditory and auditory/auditory association cortex when reading-ability is held constant indicates that neural effects of letter-speech sound integration are tightly coupled to reading performance. Thus, the present findings indicate a role for letter-speech sound integration as a foundational skill that can predict individual variations in reading performance. The results are in line with a multisensory account of dyslexia, which posits that reading difficulties manifest themselves when auditory codes for processing speech sounds have to be coupled to visual codes for processing alphabetic letters (chapter 1 ).

Yet, it may not be the knowledge about letter-speech sound associations per se that is important or reading but the instrumental use of such associations (Blomert, 2005). In chapter 2, it was shown that superior temporal and middle temporal brain regions were sensitive to the congruency between speech sounds and letters even under conditions were the visual letter was task-irrelevant. This advocates the highly automated processing of these associations in the literate brain, in line with 
previous behavioral (Dijkstra et al., 1989; Ehri and Wilce, 1980), and electrophysiological (Froyen et al., 2008) findings. Such automatic processing, in turn, has been linked to developmental progression in reading skill (Booth et al., 1999). In line with such an interpretation, the reduced neural activation to letter-speech sound pairs in dyslexia in the present experiments was associated with slower reaction times on a simple letter-speech sound matching task (chapter 3 and 4). Based on the assumption that faster processing is one of the main indices for the automation of a cognitive process (Schneider and Chein, 2003), this finding suggests that the reduced neural activation in dyslexia might relate to the less automated processing of letter-speech sound associations.

\subsubsection{DEVIANT PROCESSING OF SPEECH SOUNDS IN DYSLEXIA AND ITS RELATION TO LETTER-SPEECH SOUND INTEGRATION}

For the interpretation of our second main finding (i.e. reduced responses to speech sounds in isolation), an important result was the correlation between the auditory response to speech sounds in adult dyslexia and performance on phonological awareness tasks (chapter 3). Moreover, chapter 5 demonstrated that reduced neural activation in response to speech sounds in adult dyslexia consistently contrast dyslexic readers compared to both, a chronological-age as well as readinglevel control group. The reduced activation for speech sounds in dyslexia may therefore be linked to dyslexia per se rather than current reading ability. Reduced responses to speech sounds could be indicative of a phonological processing deficit, in line with the proposed core function of phonological processes in reading disabilities (Ramus, 2003; Vellutino, 1978; Vellutino et al., 2004). Importantly, the unisensory activation was found to be tightly linked to the strength of the multisensory integration response even if integration was defined in the absence of a direct comparison with the unisensory response (chapter 3 ). These findings indicate that the dependency between multisensory integration effects and the unisensory response is a general response characteristic in auditory and superior temporal brain regions. A critical question then is: can the reduced multisensory integration effects in dyslexia be explained through their already weaker response to unisensory presented speech sounds?

Despite the evidently important role of phonological processes in dyslexia, several aspects of the present data suggest that such a reduction of the multisensory integration responses in dyslexia to an auditory-phonological processing problem may be too simplistic. First, the data in chapter 4 show that the individual variance on reading tasks is better explained by the neural congruency response in combination with the auditory response to speech sounds than it is by the auditory response alone. Specifically, auditory effects explained no additional variance that was not already explained by the effect of congruency in PT/HS and STS, while the congruency effect explained an additional $20 \%$ of the variance in reading above and beyond the auditory response to speech. Secondly, when dyslexic adult read- 
ers were equated in their absolute reading score to a group of younger control readers (chapter 5 ) the congruency effect was found to be eliminated while the response to speech sound was not. This suggests that the multisensory congruency effect and unisensory response to speech sounds can at least partly be dissociated. Thus, while the present findings indicate that the reduced auditory response to speech sounds is important for explaining reading difficulties in dyslexia, they also suggest that the multisensory response most likely does not fully depend on the unisensory response for the process of reading.

\subsection{ON THE ABSENCE OF MULTISENSORY SUPPRESSION EFFECTS IN DYSLEXIA}

In the light of these two sets of findings (i.e., reduced activation to speech sounds in isolation and reduced activation for letter-speech sound integration), it is valuable to not only define multisensory integration through 'content-related' criteria (i.e., the congruency effect) but also through 'stimulus-related' criteria that account for the relative strength of the unisensory response (i.e. multisensory enhancement and suppression). This may provide an alternative window into the nature of integrative processes. In chapter 3 and 4, we found that fluent readers' suppressed cortical activity to incongruent letter-speech sound pairs in auditory and superior temporal brain regions relative to processing speech sounds in isolation. This suppression effect was absent or strongly reduced in dyslexia. We interpreted this result as reflecting less efficient discrimination of non-existing (incongruent) from existing (congruent) audiovisual language pairs in dyslexic subjects. From a developmental perspective, learning the alphabetic principle requires the transition from perceiving matching letter-speech sound pairs as 'unfamiliar' stimulus-combination to perceiving those same stimulus-pairs as 'familiar'. Frequency of exposure, explicit and rule-based training as well as experience in use may all be important factors to enable this perceptual transition. Another important factor may be attention to letter-sound mappings during the learning of these associations. Attention has long been assigned a key role for the learning of new cognitive skills (Huey, 1908) and, interestingly, has also been implicated in the progression from effortful to fluent automatic reading (La Berge and Samuels, 1974; for review Shaywitz and Shaywitz, 2008). Hence, a failure of dyslexic readers to differently process congruent vs. incongruent letter-speech sound pairs may be the result of a altered learning process, where insufficient levels of attention are devoted to congruent as opposed to incongruent audiovisual stimuli. This is supported by findings from behavioral studies suggesting that children with dyslexia suffer from visual and auditory deficits in automatic capture of spatial attention that might distort the development of phonological and orthographic representations (Facoetti et al., 2003; Facoetti et al., 2009). Consequently, a possible interpretation of the present suppression findings in fluent readers (chapter 3 and 4 ) is that they 
represent a neural mechanism for preventing the binding of irrelevant audiovisual associations during learning to read.

As opposed to the differences in multisensory suppression, multisensory enhancement effects were not a prominent finding in the present experiments. In chapter 3 and 4, we did not find multisensory enhancement effects for fluent nor dyslexic readers. In study 2, we found enhancement in a more liberal post-hoc analysis of the congruency contrast, but not when a more strict statistical analysis approach was employed (i.e., congruency-by-noise interaction). Based on findings from monkey electrophysiological work (Stanford et al., 2005) we previously suggested that the absence of enhancement effects for congruent letter-speech sound pairs in fluent readers may relate to a simple constraint of the $\mathrm{FMRI} B O L D$ response, where fairly strong responses to unisensory auditory stimulation in the present tasks increased the likelihood for finding multisensory suppression at the cost of finding multisensory enhancement effects. The intermittent finding of enhancement effects in chapter 2 here and in previous studies on letter-speech sound integration in literate adults (Van Atteveldt et al., 2004), may be a consequence of the different statistical and analytic methods.

\subsection{THE INFLUENCE OF READING EXPERIENCE IN DYSLEXIA}

Another potentially useful strategy to better understand the contribution of letterspeech sound integration vs. speech processing in dyslexia may be to look at the influence of reading experience across the child and adult dyslexia studies (study 4 vs. 3). While the congruency effects in the child study (chapter 4) were highly correlated with performance several direct and indirect measures of reading, the congruency effects in the adult study (chapter 3 ) were only weakly related to those behavioral measures. Here, the neural response to speech sounds strongly correlated with performance on reading-related behavioral tasks. Interestingly, it was also the response to speech sounds that was not eliminated by comparing the adult dyslexic group to a group of younger control children (chapter 5), indicating that the auditory response to speech sounds represents the core deficit in dyslexia. A possible explanation for the weaker correlations between reading-related behavioral measures and $\mathrm{fMRI}$ responses indexing letter-speech sound integration in adult dyslexic readers may be that letter-speech sound integration impairments can either partly be compensated for by reading experience or get masked by the use of compensatory strategies, while a deficit in processing speech sound cannot. Conversely, the finding that beginning dyslexic readers exhibited strong correlations between the neural congruency effect and reading-related behavioral measures may reflect the fact that children still rely more directly on letter-speech sound associations during reading than adult readers. Nine year old children are still close to the start of alphabetic reading instruction and their letter-speech sound association skills are likely to be not yet fully developed (Blomert and Vaessen, 2009). Importantly, the fact that letter-speech sound integration is more 
closely related to behavioral reading measures in dyslexic children compared to dyslexic adults suggests an important role for multisensory integrative processes at the beginning of reading instruction. Conversely, the finding that activation for speech sounds is related to reading-related measures in adult dyslexia suggests a more fundamental role in reading that may not be dependent on the exact stage of the learning process.

The fact that dyslexic children vs. adults underactivate slightly different brain structures for processing letters, speech sounds and their combination could also be taken as an index for a different role of these processes in reading (see 6.1). For example, the fact that reduced activation in STS for integrating letters and speech sounds was a factor relevant for dyslexic children but not for dyslexic adults suggests a functional role of STS in establishing letter-speech sound associations important for learning to read. This would be in line with recent fMRI results, in which STS was assigned a general role in the creation of audiovisual associations (Tanabe et al., 2005). Similarly, the finding that FG was a factor relevant for dyslexic children but not for dyslexic adults could be interpreted to indicate weaker specialization for processing visual orthographic inputs in dyslexic children, presumably related to children's reduced experience with print (Maurer et al., 2007). In contrast, the finding that auditory/auditory association cortex is underactivated in dyslexic children and remains underactivated in dyslexic adults suggests a fundamental role of these brain regions in the development of fluent reading skills. In sum, the present experiments suggest several -potentially relevant- differences in the neural processing of letters and speech sounds between adult and child dyslexics. Direct statistical comparisons between dyslexic groups varying in reading experience, however, are necessary to firm up those suggestions.

\subsection{POTENTIAL INFLUENCES OF TASK-DEMANDS}

A brief comment should be made on the potential influences of task demands on the observation of auditory and letter-speech sound integration effects in dyslexia. Although systematically exploring the influence of task demands was not the purpose of the present studies (for such evidence see van Atteveldt et al., 2007), there are several advantages as well as disadvantages associated with the choice of the current (pseudo-)passive task designs for studying letter-speech sound integration in dyslexia (study 3 to 5). The advantage is that by using such simple tasks we may have tapped into more basic perceptual aspects of processing than previous investigations, which have commonly used rather complex phonological tasks such as rhyming (Cao et al., 2006; Temple et al., 2001) or pseudoword reading (Brunswick et al., 1999; Horwitz et al., 1998; Paulesu et al., 2001; Rumsey, 1992; Temple, 2002). One potential problem with the use of complex tasks is that dyslexic readers are by definition poor readers and their performance on reading tasks is more variable than that of control subjects. As a result, in-scanner performance is easily confounded by task factors, a problem that scales with the task difficulty (Schlaggar 
et al., 2002; Schlaggar and McCandliss, 2007). Such arguments support the choice of the present (pseudo-)passive designs for studying dyslexia in chapter 3 and 4. Moreover, the result from chapter 2 that letter-speech sound integration in the brain of literate adults can still be observed under active task conditions, argue for the feasibility of passive task designs as a means to reveal behaviorally-relevant neural integration. However, a disadvantage associated with the present designs is that they provide no direct control for attention-related effects on neural activity during scanning (chapter 3 to 5). Although such effects should be weaker than, for example, in reading tasks because of the simple nature of the stimuli, the possibility exists that some of the effects we observed can be explained through reduced capture of attention to those stimuli during scanning in the dyslexic group. The problem is that such attention effects may either reflect functionally relevant phenomena related to the clinical picture of dyslexia or simply an 'unwanted' confounding variable during scanning. While the first option may be apt and potentially compelling (also see 6.2), we consider a strong interpretation in terms of a general attentional confound in the present experiments unlikely. Aspects that speak against such an interpretation include the absence of differential activity in frontal-parietal attention networks (chapter 3 and 4), overall similar dynamic ranges of the BOLD responses in dyslexic and control subjects (aside from those regions that were differentially involved, e.g., see chapter 4), and equally accurate as well as equally fast responses to primary-task stimuli (auditory, visual and audiovisual) in subjects with and without dyslexia (chapter 4).

\subsection{A FRAMEWORK FOR THE STUDY OF DYSLEXIA: MULTISENSORY, UNISENSORY, OR BOTH?}

An important theoretical question that arises from the overall findings presented in this thesis is what role neural processes related to the multisensory integration of letters and speech sounds vs. those related to the processing of unisensory speech sounds have in explaining reading failure in dyslexia. The answer to this question determines what a multisensory approach can add to our current knowledge about dyslexia.

In chapter 1 it was argued that a multisensory framework may be crucial for the study of reading and reading disabilities and that the basic problem causing reading failure in dyslexia manifests itself when auditory codes for processing phonemes have to be coupled to visual codes for processing letters. Clearly, the present results are in line with such a multisensory account in that deviant neurofunctional processing of letter-speech sound integration is shown to be linked to poor reading skills. At the same time, however, the present findings emphasize the role of phonological processes in explaining reading difficulties. The fact that dyslexia is associated with impairments in phonological processing is a well established finding (for review see Vellutino et al., 2004). The unique aspect of the present experi- 
ments is that they may allow connecting this phonological deficit to the actual process of reading.

From a developmental perspective, becoming a skilled reader has been associated with both, the developmental of phonemic awareness skills and the development of letter-speech sound associations (Ehri, 2005). As discussed in chapter 1, a child's awareness for the isolated phonemes in spoken language might develop in response to reading instruction, while at the same time, phonemic awareness fosters the acquisition of reading skills (Ehri, 1992; Ehri, 2005; Perfetti et al., 1987; Torgesen et al., 1994; Wagner and Torgesen, 1987; Ziegler and Goswami, 2005). In line with this, the present experiments revealed evidence for both, a phonemic and a letter-speech sound integration deficit in dyslexia (chapter 3 to 5). The interactive nature of reading development and the development of phonemic awareness skills prohibit a strong interpretation of the present findings in terms of 'cause' and 'effect'. Instead, the reduced neural unisensory responses to speech sounds and reduced letter-speech sound integration in dyslexia may both better be conceptualized as arising through a process of deviant interactive specialization, in which neural structures such as superior temporal or auditory cortex get insufficiently tuned to combine orthographic and phonological inputs to support skilled reading. Both effects seem to have an important, but different role in learning to read and reading failure. In chapter 5 , we used two sets of comparisons (i.e., chronologicalage and reading level comparisons) to examine which neural processes are related to dyslexia itself and which are only related to its behavioural manifestation, i.e. reading ability. The results demonstrated that reduced letter-speech sound integration in dyslexia relates to current reading ability, while the reduced activation to speech sounds in dyslexia is a fundamental weakness that cannot be reduced to differences in current reading level (chapter 5). The connection between a phonological core deficit in dyslexia and the actual reading problem (i.e. the fast identification of visual words) may best be viewed as a continuous developmental path, which makes it possible to interpret the present findings as follows: the neural integration of letters and speech sounds may be situated proximate to the actual reading problem because it reflects the translation between spoken language development and the development of actual reading skills. In that, the present results may help to solve a longstanding theoretical question in reading research, namely how a deficit in the auditory-phonological domain translates into a problem in the fast recognition of visual words.

The neural processing of speech sounds, in contrast, might be more distal to the actual process of reading in that it cannot directly explain why dyslexia manifests itself as a problem in visual word recognition. At the same time, however, phonological processes might be more 'causal' for explaining reading failure. Yet, they shape and get shaped through the acquisition of reading skills.

A coherent understanding of the neurobiology of dyslexia is likely to require the integration of both, distal and proximate explanations. One of the most important steps in this respect may be longitudinal developmental studies that allow to 
determine how phonological processes and reading acquisition (e.g., knowledge about letters, vocabulary growth) interact and how this is reflected in the differential recruitment and connectivity of relevant brain regions. 


\section{REFERENCES}

Blomert L. Dyslexie in Nederland. Amsterdam: Uitgeverij Nieuwezijds, 2005.

Blomert L, Vaessen A. 3DM Differential Diagnostics for Dyslexia: Cognitive Analysis of Reading and Spelling. Amsterdam: Boom Test Publishers, 2009.

Booth JR, Perfetti CA, MacWhinney B. Quick, automatic, and general activation of orthographic and phonological representations in young readers. Dev Psychol 1999; 35: 3-19.

Brunswick N, McCrory E, Price CJ, Frith CD, Frith U. Explicit and implicit processing of words and pseudowords by adult developmental dyslexics: A search for Wernicke's Wortschatz? Brain 1999; 122 ( Pt 10): 1901-17.

Cao F, Bitan T, Chou TL, Burman DD, Booth JR. Deficient orthographic and phonological representations in children with dyslexia revealed by brain activation patterns. J Child Psychol Psychiatry 2006; 47: 1041-50.

Dijkstra T, Schreuder R, Frauenfelder UH. Grapheme context effects on phonemic processing. Language and Speech 1989; 32: 98-108.

Ehri LC. Reconceptualizing the development of sight word reading and its relationship to recoding. In: Gough LE, Ehri LC and Treiman R, editors. Reading Acquisition. Hillsdale, NJ: Erlbaum Publishers, 1992.

Ehri LC. Development of sight word reading:phases and findings. In: Snowling MJ and Hulme C, editors. The science of reading: a handbook. Oxford: Blackwell Publishing, 2005: 135-45.

Ehri LC, Wilce LS. The influence of orthography on readers' conceptualization of phonemic structure of words. Applied Psycholinguistics 1980: 371-85.

Facoetti A, Lorusso ML, Paganoni P, Cattaneo C, Galli R, Umilta C, et al. Auditory and visual automatic attention deficits in developmental dyslexia. Brain Res Cogn Brain Res 2003; 16 : 185-91.

Facoetti A, Trussardi AN, Ruffino M, Lorusso ML, Cattaneo C, Galli R, et al. Multisensory Spatial Attention Deficits Are Predictive of Phonological Decoding Skills in Developmental Dyslexia. J Cogn Neurosci 2009.

Froyen D, Van Atteveldt N, Bonte M, Blomert L. Multisensory enhancement of the MMN to speech-sounds indicates early and automatic integration of letters and speech-sounds. Neurosci Lett 2008; 430: 23-8.

Horwitz B, Rumsey JM, Donohue BC. Functional connectivity of the angular gyrus in normal reading and dyslexia. Proc Natl Acad Sci U S A 1998; 95: 8939-44.

Huey E. The psychology and pedagogy of reading. New York: Macmillan, 1908.

La Berge D, Samuels SJ. Towards a theory of automatic information processing in reading. Cognitive Psychology 1974; 6: 293-323.

Maisog JM, Einbinder ER, Flowers DL, Turkeltaub PE, Eden GF. A meta-analysis of functional neuroimaging studies of dyslexia. Ann N Y Acad Sci 2008; 1145: 237-59.

Maurer U, Brem S, Bucher K, Kranz F, Benz R, Steinhausen HC, et al. Impaired tuning of a fast occipito-temporal response for print in dyslexic children learning to read. Brain 2007; 130 : 3200-10.

Paulesu E, Demonet JF, Fazio F, McCrory E, Chanoine V, Brunswick N, et al. Dyslexia: cultural diversity and biological unity. Science 2001; 291: 2165-7.

Perfetti CA, Beck I, Bell L, Hughes C. Phonemic knowldege and learning to read are reciprocal: A longitudinal study of first grade children. Merill-Palmer Quarterly 1987; 33: 283-319.

Ramus F. Developmental dyslexia: specific phonological deficit or general sensorimotor dysfunction? Curr Opin Neurobiol 2003; 13: 212-8.

Rumsey JM. Failure to activate left temporoparietal cortex in dyslexia. Archives of Neurology 1992; 49: 527-534. 
Rumsey JM, Nace K, Donohue B, Wise D, Maisog JM, Andreason P. A positron emission tomographic study of impaired word recognition and phonological processing in dyslexic men. Arch Neurol 1997; 54: 562-73.

Schlaggar BL, Brown TT, Lugar HM, Visscher KM, Miezin FM, Petersen SE. Functional neuroanatomical differences between adults and school-age children in the processing of single words. Science 2002; 296: 1476-9.

Schlaggar BL, McCandliss BD. Development of neural systems for reading. Annu Rev Neurosci 2007; 30: 475-503.

Schneider W, Chein JM. Controlled and automatic processing: Behavior, theory and biological mechanisms. Cognitive Science 2003; 27: 525-559.

Shaywitz SE, Shaywitz BA. Paying attention to reading: The neurobiology of reading and dyslexia. Development and Psychopathology 2008; 20: 1329-1349.

Stanford TR, Quessy S, Stein BE. Evaluating the operations underlying multisensory integration in the cat superior colliculus. J Neurosci 2005; 25: 6499-508.

Tanabe HC, Honda M, Sadato N. Functionally segregated neural substrates for arbitrary audiovisual paired-association learning. J Neurosci 2005; 25: 6409-18.

Temple E. Brain mechanisms in normal and dyslexic readers. Curr Opin Neurobiol 2002; 12: 17883.

Temple E, Poldrack RA, Salidis J, Deutsch GK, Tallal P, Merzenich MM, et al. Disrupted neural responses to phonological and orthographic processing in dyslexic children: an fMRI study. Neuroreport 2001; 12: 299-307.

Torgesen JK, Wagner RK, Rashotte CA. Longitudinal studies of phonological processing and reading. J Learn Disabil 1994; 27: 276-86; discussion 287-91.

van Atteveldt N, Formisano E, Goebel R, Blomert L. Integration of letters and speech sounds in the human brain. Neuron 2004; 43: 271-82.

van Atteveldt NM, Formisano E, Goebel R, Blomert L. Top-down task effects overrule automatic multisensory responses to letter-sound pairs in auditory association cortex. Neuroimage 2007; 36: 1345-60.

Vellutino FR. Dyslexia: Theory and Research. Cambridge: MIT Press, 1978.

Vellutino FR, Fletcher JM, Snowling MJ, Scanlon DM. Specific reading disability (dyslexia): what have we learned in the past four decades? J Child Psychol Psychiatry 2004; 45: 2-40.

Wagner R, Torgesen J. The nature of phonological processes and its causal role in the acquisition of reading skills. Psychological Bulletin 1987; 101: 192-212.

Ziegler JC, Goswami U. Reading acquisition, developmental dyslexia, and skilled reading across languages: a psycholinguistic grain size theory. Psychol Bull 2005; 131: 3-29. 
Learning to read is an important milestone in individual cognitive development. In alphabetic languages, the first and critical step for becoming a skilled reader is to learn the association between visual letters and auditory units of speech (speech sounds). Understanding the neurobiological mechanisms that subserve the learning of letter-speech sound associations is therefore an important factor to explain reading and reading failure. The experiments presented in this thesis employ functional magnetic resonance imaging ( $f M R I)$ to investigate the neural mechanisms subserving the integration of letters and speech sounds in skilled readers (chapter 2) as well as adults and children diagnosed with developmental dyslexia (chapter 3 to 5).

Chapter 2 assessed whether previously-reported brain activation paradigms contrasting congruent and incongruent letter sound integration influence perceptual decisions about speech sounds, and thus whether letter-speech sound integration is a behaviorally relevant phenomenon. Superior temporal and middle temporal brain regions were found to be sensitive to the congruency between speech sounds and letters, and behavioral decisions about the identity of speech sounds were faster and more accurate when those sounds were paired with congruent as opposed to incongruent letters. Moreover, it was found that parametric variations in the quality of the visual letter affected behavioral responses to speech sounds as well as neural responses to those sounds. Notably, this effect was found in the absence of explicit requirements to process the visual letters, indicating that letterspeech sound integration is highly automated in the literate adult brain. This finding validates and extends observations from previous studies in healthy adults showing letter-speech sound integration is behaviorally relevant and not an epiphenomenon of presenting those stimuli during scanning.

Based on these and previous findings of letter-speech sound integration in literate adults, Chapter 3 then examined the role of letter-speech sound integration for explaining reading failure in developmental dyslexia. Adult dyslexic readers exhibited reduced responses for processing congruent vs. incongruent letter-speech sound pairs in superior temporal gyrus just anterior of early auditory cortex. Moreover, dyslexic readers exhibited weaker responses to speech sounds presented in isolation, potentially indicating deviant processing of phonological inputs. This effect was positively correlated with performance on phonological tasks. Furthermore, the reduced integration effects in STG of the dyslexic group remained, even after degree of auditory activation was accounted for. This was reflected in a lack of multisensory suppression for the processing of incongruent letter-speech sound pairs and suggests the existence of two functionally distinct neural deficits. One central question that emerged from this study was whether a neural deficit in integrating letters and speech sounds in adult dyslexia is a fundamental factor to 
explain reading difficulties or whether it is merely a reflection of a lifetime of reading struggle in dyslexic adults.

Chapter 4 therefore examined whether beginning readers (second grade of elementary school) show a neural deficit in integrating letters and speech sounds in the brain regions previously implicated in adult dyslexia. The results revealed that dyslexic children show reduced activity for the processing of congruent vs. incongruent letter-speech sound pairs in auditory/auditory association cortex and superior temporal sulcus. The degree of this effect correlated with performance on reading-related tasks indicating its relevance for reading success. Unlike adult dyslexics, however, dyslexic children showed reduced unisensory responses to letters in fusiform gyrus as well as reduced activity for processing speech sounds in anterior superior temporal gyrus/sulcus as well as auditory/ auditory association cortex. Remarkably, the neural signals alone, when combining integration responses in the auditory cortex with neural responses to visual letters in FG, were able to explain $40 \%$ of the variance in individual reading performance assessed within standardized tests. Together the child and adult findings argue against the notion that letter-speech sound integration deficits develop over a lifetime of reading struggle. Instead, the results support the view that letter-speech sound integration is an emergent property of learning to read that develops inadequately in dyslexic readers.

Finally, Chapter 5 investigated whether the previously observed differences in neural integration between dyslexic and control readers (chapter 3 and 4) reflect a developmental delay in reading skill acquisition or a deficit related to dyslexia itself. The main finding of this chapter was that underactivation in auditory/auditory association cortex for the integration of letters and speech sound in adult dyslexia is related to current reading level rather than being a specific deficit in dyslexia. Reduced activation for processing speech sounds in isolation, in contrast, seemed to reflect a dyslexia-specific deficit rather than simply a developmental delay accounted for by current reading ability.

In sum, the experiments in this thesis make a strong case for the existence of deviant letter-speech sound integration as a proximate cause of poor reading skills in dyslexia. At the same time, they support the notion that the core deficit in dyslexia may be situated in the phonological domain. Both explanations might ultimately be germane to a complete understanding of the neurobiology of reading and reading failure. 


\section{SAMENVATTING}

Leren lezen is een mijlpaal in de cognitieve ontwikkeling van een individu. In alfabetische talen wordt het leren van de associaties tussen enkele letters en klanken gezien als een belangrijke eerste stap in de leesontwikkeling. Kennis over de neurobiologische mechanismen, die aan deze vaardigheid ten grondslag liggen is daarom een cruciale stap voor het begrijpen van de normale en verstoorde leesontwikkeling, zoals bij mensen met dyslexie. De doelstellingen van het in dit proefschrift gepresenteerde onderzoek was om met behulp van functionele hersenscans (functional magnetic resonance imaging, $\mathrm{fMRI}$ ) onze kennis over de neurale basis van het verwerken van letter-klank associaties te verdiepen, zowel bij normale lezers (hoofdstuk 2) als ook bij volwassenen en kinderen met dyslexie (hoofdstuk 3 $\mathrm{t} / \mathrm{m}$ 5).

In Hoofdstuk 2 wordt onderzocht of eerder beschreven congruentie effecten bij het verwerken van letter-klank associaties in de hersenen de expliciete beslissingen met betrekking tot de identiteit van een klank kunnen beïnvloeden, en dus of deze congruentie effecten een relevantie hebben voor het gedrag van een proefpersoon. De resultaten wijzen uit dat de 'superior temporal' en 'middle temporal' hersengebieden gevoelig zijn voor de congruentie tussen letters en klanken, en dat ook op gedragsniveau de identificatie van spraakklanken sneller en nauwkeuriger verloopt wanneer er gelijktijdig een congruente in plaats van een incongruente letter wordt aangeboden. Daarnaast wees het onderzoek uit dat parametrische variatie in de kwaliteit van de letter zowel de hersenactiviteit als de klankidentificatie systematisch beïnvloeden. Het feit dat deze effecten gevonden zijn met een taak waarin proefpersonen niet expliciet op de letters moesten letten duidt op een automatische integratie van letter en klank informatie in de hersenen van normaal lezende volwassenen. Deze bevindingen valideren en vernieuwen eerdere resultaten omdat ze aantonen dat de integratie van letter-klank informatie in de hersenen relevant is voor ons gedrag en niet alleen een epifenomeen van het presenteren van letter-klank stimuli tijdens de scan.

Gebaseerd op de bevindingen uit hoofdstuk 2 en eerdere resultaten over letterklank integratie, is in hoofdstuk 3 onderzocht in hoeverre afwijkingen in hersenprocessen gerelateerd aan letter-klank integratie een verklaring kunnen leveren voor leesproblemen bij dyslexie. Uit de data bleek dat volwassen dyslectici minder hersenactiviteit vertonen voor het verwerken van congruente vs. incongruente letter-klank paren in de 'superior temporal gyrus', anterior ten opzichte van de vroege auditieve cortex. Ook vertonden dyslectische lezers minder activiteit in respons op spraaklanken alleen, wat erop duidt dat dyslectici moeilijkheden hebben met het verwerken van dit soort fonologische informatie. Het herseneffect correleerde met hun prestaties op fonologische gedragstaken. Bovendien bleef de verminderde activiteit voor letter-klank integratie bestaan ook toen voor de abso- 
lute hoeveelheid auditieve activiteit gecontroleerd werd. Dit werd aangetoond door de afwezigheid van een multisensorisch suppressie effect voor het verwerken van incongruente letter-klank stimuli in de dyslexie groep en wijst op het bestaan van twee, functioneel gescheiden, neurale deficits. Een kritische vraag die de bevindingen uit hoofdstuk 3 opriepen was of het integratie deficit leesproblemen in dyslexie kon verklaren of dat het alleen een effect is van een heel leven slecht kunnen lezen bij dyslectische volwassenen.

Om deze vraag te onderzoeken, wordt in hoofdstuk 4 nagegaan of ook beginnende lezers (groep 5/6) al een neuraal deficit in de integratie van letter-klank informatie vertonen. De data laten zien dat dyslectische kinderen verminderende hersenactiviteit vertonen voor het verwerken van congruente vergeleken met incongruente stimuli in de auditieve/auditieve associatie cortex en de 'superior temporal sulcus'. De sterkte van dit effect was gecorreleerd met de prestatie op leesgerelateerde taken, en geeft dus een indicatie van de relevantie van dit proces. Anders dan volwassen dyslectici, vertoonden kinderen ook verminderde hersenactiviteit voor het verwerken van letters in de 'fusiform gyrus' en het verwerken van spraakklanken in het anterieure gedeelte van de 'superior temporal gyrus/sulcus' en de auditieve cortex. Voornamelijk de hersensignalen voor letter-klank integratie in de auditieve cortex en de signalen voor visuele letters in de 'fusiform gyrus' verklaarden bijna $40 \%$ van de variatie in individuele leesscores. Samen genomen spreken de volwassen en kinderdata tegen het idee dat een deficit in letter-klank integratie het resultaat is van een heel leven moeilijk lezen. Deze resultaten ondersteunen eerder de theorie dat letter-klank integratie tijdens het leren lezen ontwikkelt en dat deze ontwikkeling onvoldoende verloopt bij dyslectische kinderen.

Ten slotte werd in hoofdstuk 5 nagegaan of de eerder beschreven verschillen in de neurale integratie van letters en klanken tussen dyslectisch en normale lezers (hoofdstukken 3 en 4) het resultaat zijn van een vertraagde ontwikkeling in leesontwikkeling of een tekort dat rechtstreeks verbonden is aan het klinische beeld van dyslexie. De bevinden wijzen uit dat de verminderde activiteit in de auditieve/auditieve associatie gebieden voor de integratie van letter-klank informatie in volwassen dyslectici meer te maken hebben met het leesniveau dan met dyslexie zelf. Aan de andere kant wijzen de resultaten er ook op dat minder hersenactiviteit voor het verwerken van spraakklanken alleen een tekort is dat als dyslexie-specifiek beschouwd kan worden en niet slechts als een vertraging in het normale ontwikkelingspatroon.

Samenvattend kan gesteld worden dat de onderzoeken in deze proefschrift laten zien dat een afwijkende integratie van letter-klank informatie in de hersenen een mogelijk 'proximale' oorzaak is van slecht lezen bij mensen met dyslexie. Even goed bieden de uitkomsten steun voor het idee dat de oorzaak van leesproblemen in de fonologische domain te vinden is. Beide verklaringen blijken relevant voor een compleet begrip van leren lezen en leesproblemen. 


\section{ACKNOWLEDGEMENTS}

I would like to gratefully acknowledge my promoters, Prof Dr. Leo Blomert and Prof Dr. Rainer Goebel for their invaluable advice and support during the past four years. Leo, you made this $\mathrm{PhD}$-project possible through your persistent vision for the direction dyslexia research should take, and provided critical pragmatic guidance in seeing this through to completion. Rainer, your constant enthusiasm for the wonders of brain imaging is highly contagious and will be something I will take with me on future scientific adventures.

In addition, I would like to thank Nienke van Atteveldt and Elia Formisano for their guidance and 'getting me started'. Your help has been priceless to get me through the initial complexities of my PhD project. A very special thanks goes to Nienke, who took on the role of my daily mentor from the very start. I highly appreciate your willingness to share your experience and knowledge with me!

Thanks to Prof. Dr. Peter de Weerd, Dr. Alex Sack, Dr. Markus Naumer and Prof. Dr. Jean Vroomen for being part of the reading committee. Furthermore, I would like to thank all subjects, especially the children and parents who have participated in these studies and made this research possible, the Regional Institute of Dyslexia in Arnhem and Maastricht for their help with subject recruitment, as well as the Koninklijke Nederlandse Militaire School (KNMS) in Weert for working with us on the adult dyslexia project.

I am also indebted to my wonderful 'dyslexic' colleagues: Dries, Jeannette, Gonny, Anniek and -at certain times- Lilian, Jochen, Hanne and Anke, as well as the extended lunch/party version including Petra, Teresa, Fede, and Noel. Zonder jullie waren de afgelopen jaren nooit zo gezellig geweest! It was great how I could always just storm into anyone's room to share joy and frustrations about work- united we stand! Dries, I am glad you have become my 'scientific buddy' to discuss ideas, results and (im?) possible interpretations. Jeannette, you joined late and Jochen, you left early, but I am happy that I met you. Nienke and Amanda, thanks for being wonderful roomies. Betty, thanks you for your friendship through all those years, lots of laughter, talk, support and simply good times. It wouldn't have been the same without you. Lars and Teresa: you know how it all started- I am glad you stayed around and gave me a sense of being grounded. Fraukje, Ralph, Pim, Deliane, Maedels!-you are a big part of work-life balance, I am lucky to know you.

Numerous colleagues of the NC department, Brain Innovation and the secretary's office - you all contributed to a productive and creative working environment and provided support wherever I needed it. Special thanks goes to Annemie for efficiently taking care of many administrative issues, to Armin for his friendly and patient support on BV issues, as well as to Bernadette and Elia as our 'capgroepvoorzitters'. 
Liebe Familie (Mama, Peter, Papa, Sandra \& Elena): Danke für eure bedingungslose Unterstützung in all den Jahren, euer Vertrauen und Interesse. Papa: danke für stundenlanges Zuhören und die Fähigkeit im richtigen Moment die richtige Frage zu stellen, für die Disziplin mit der du Zeitungsausschnitte über psychologische Forschung für mich ausschneitest, und natürlich deinen persönlichen Anekdoten über den Sinn und Unsinn der Wissenschaft. Mama, danke für deine pragmatische Unterstützung in allen Lebenslagen, deine Fürsorge und wertvollen Kommentaren wie: „Ja und, was bringt das?“ oder „Dann mach halt was anderes!“.

Lastly, there is one more person to thank. Bruce, without you sharing my joy and despair, without your patience, support and humorous pep-talk these past years would not have been the same........ am deeply grateful for your friendship and love. 


\section{CURRICULUM VITAE}

Vera Blau, born August 27th 1978 in Munich, Germany, started studying psychology at Maastricht University in 2001 and completed her bachalors in 2002. She then attended the track Biological Psychology with a specialization in the field of Cognitive Neuroscience. After working as a research assistant in Maastricht and a research internship at the Sackler Institute for Developmental Psychobiology in New York, she received her master's degree in psychology from Maastricht University in 2004. She then continued her work as a PhD student at the department of Psychology and Neuroscience in Maastricht under the supervision of Prof. Dr. L. Blomert and Prof. Dr. R. Goebel. She recently received a Rubicon fellowship from the Dutch Organisation for Scientific research (NWO), on which she will continue her work as a post-doctoral researcher at Vanderbilt University in the United States.

Contact Information:

Vera Blau

7110 MRB III BioSci Bldg

465 21st Ave South

Nashville, TN 37232

vera.c.blau@vanderbilt.edu 